\title{
Character bounds for finite groups of Lie type
}

\author{
by \\ ROMAN BEZRUKAVNIKOV \\ Massachusetts Institute of Technology \\ Cambridge, MA, U.S.A. \\ Aner Shalev \\ Hebrew University \\ Jerusalem, Israel \\ MARTIN W. LIEBECK \\ Imperial College \\ London, U.K. \\ Pham Huu Tiep \\ Rutgers University \\ Piscataway, NJ, U.S.A.
}

\section{Contents}

1. Introduction . . . . . . . . . . . . . . . . . . . 2

2. Character bounds: Proof of Theorem 1.1 . . . . . . . . . . . 13

3. General and special linear groups . . . . . . . . . . . . . . . 23

3.1. Proof of Theorem $1.4 \ldots \ldots \ldots \ldots \ldots$

3.2. Proof of Theorem $1.3 \ldots \ldots \ldots \ldots 24$

3.3. Elements with extension-field centralizers . . . . . . . . . 26

3.4. Unipotent elements in general linear groups . . . . . . . . . 30

3.5. Special linear groups . . . . . . . . . . . . . . . . . . . . . . . . . . . . . . . . . . 32

3.6. Proof of Theorem $1.5 \ldots \ldots \ldots \ldots$

4. Bounds for the constant $\alpha(\mathcal{L})$ : Proofs of Theorems 1.6, 1.7 and 1.1035

4.1. Case $\mathcal{G}=\mathrm{GL}_{n}(\mathbb{K})$ or $\mathcal{G}=\mathrm{SL}_{n}(\mathbb{K}) \ldots \ldots \ldots \ldots \ldots$

4.2. Symplectic groups . . . . . . . . . . . . . . . . . . . . . . . . 38

4.3. Orthogonal groups . . . . . . . . . . . . . . . . . . . . 41

4.4. Exceptional groups: Proof of Theorem $1.7 \ldots \ldots$. . . . . . . 44

4.5. Proof of Corollary 1.8 and Theorem $1.9 \ldots \ldots \ldots$

4.6. Bounds for $\mathcal{G}=\mathrm{GL}_{n}(q)$ : Proofs of Theorem 1.10 and Corol-

lary $1.11 \ldots \ldots \ldots \ldots \ldots \ldots$

5. Random walks . . . . . . . . . . . . . . . . . . . . . . . 51

Corollaries 1.13 and $1.14 \ldots \ldots \ldots \ldots \ldots$

References ............................ 55

The first author was partially supported by the NSF grants DMS-1102434 and DMS-1601953. The second and third authors acknowledge the support of EPSRC grant EP/H018891/1. The third author acknowledges the support of ERC advanced grant 247034, ISF grants 1117/13 and 686/17, BSF grant 2016072 and the Vinik chair of mathematics which he holds. The fourth author was partially supported by the NSF grants DMS-1839351 and DMS-1840702, the Simons Foundation Fellowship 305247, the EPSRC, and the Mathematisches Forschungsinstitut Oberwolfach. 


\section{Introduction}

For a finite group $G$, a character ratio is a complex number of the form $\chi(g) / \chi(1)$, where $g \in G$ and $\chi$ is an irreducible character of $G$. Upper bounds for absolute values of character values and character ratios have long been of interest, for various reasons; these include applications to random generation, covering numbers, mixing times of random walks, the study of word maps, representation varieties and other areas. For example, character ratios are connected with the well-known formula

$$
\frac{1}{|G|} \prod_{i=1}^{k}\left|C_{i}\right| \sum_{\chi \in \operatorname{Irr}(G)} \frac{\chi\left(c_{1}\right) \ldots \chi\left(c_{k}\right) \chi\left(g^{-1}\right)}{\chi(1)^{k-1}}
$$

expressing the number of ways of writing an element $g \in G$ as a product $x_{1} x_{2} \ldots x_{k}$ of elements $x_{i} \in C_{i}$, where $C_{i}=c_{i}^{G}$ are $G$-conjugacy classes of elements $c_{i}, 1 \leqslant i \leqslant k$, and the sum is over the set $\operatorname{Irr}(G)$ of all irreducible characters of $G$ (see $[2,10.1]$ ). This connection is sometimes a starting point for such applications; it has been particularly exploited for almost simple (or quasisimple) groups $G$.

Another classical formula involving character ratios goes back to Frobenius in 1896 [10]. It asserts that, for any finite group $G$, the number $N(g)$ of ways to express an element $g \in G$ as a commutator $[x, y], x, y \in G$, satisfies

$$
N(g)=|G| \sum_{\chi \in \operatorname{Irr}(G)} \frac{\chi(g)}{\chi(1)} .
$$

This formula is widely used, and served (together with character bounds) as an important tool in the proof of Ore's conjecture [30].

We are particularly interested in the so-called exponential character bounds, namely bounds of the form

$$
|\chi(g)| \leqslant \chi(1)^{\alpha_{g}}
$$

sometimes with a multiplicative constant, holding for all characters $\chi \in \operatorname{Irr}(G)$, where $0 \leqslant \alpha_{g} \leqslant 1$ depends on the group element $g \in G$. Obviously, if $g$ is central in $G$, then we must have $\alpha_{g}=1$, but for most elements $g$ we aim to find $\alpha_{g}<1$ which is as small (and explicit) as possible. One advantage of exponential character bounds is that they imply the inequality

$$
\left|\frac{\chi(g)}{\chi(1)}\right| \leqslant \chi(1)^{-\left(1-\alpha_{g}\right)},
$$

so the upper bound on the character ratio becomes smaller as the character degree grows.

The first exponential character bound was established in 1995 for symmetric groups $\mathrm{S}_{n}$ by Fomin and Lulov [9]. They show that, for permutations $g \in \mathrm{S}_{n}$ which are products 
of $n / m$ cycles of length $m$ and for all characters $\chi \in \operatorname{Irr}\left(\mathrm{S}_{n}\right)$ we have

$$
|\chi(g)| \leqslant c(m) n^{1 / 2-1 / 2 m} \chi(1)^{1 / m}
$$

for a suitable function $c: \mathbb{N} \rightarrow \mathbb{N}$.

In [35] this bound and some extensions of it were applied in various contexts, including the theory of Fuchsian groups. Subsequently, exponential character bounds which hold for all permutations $g \in \mathrm{S}_{n}$ and which are essentially best possible were established in 2008 in [25], with applications to a range of problems: mixing times of random walks, covering by powers of conjugacy classes, as well as probabilistic and combinatorial properties of word maps.

Can we find good exponential character bounds for groups of Lie type? This problem has turned out to be quite formidable; it has been considered by various researchers over the past two decades, and various approaches have been attempted, but it is only in this paper that strong (essentially best possible) such bounds are established.

The first significant bound on character ratios for groups of Lie type was obtained in 1993 by Gluck [13], who showed that $|\chi(g)| / \chi(1) \leqslant C q^{-1 / 2}$ for any non-central element $g \in G(q)$, a group of Lie type over $\mathbb{F}_{q}$, and any non-linear irreducible character $\chi$ of $G(q)$, where $C$ is an absolute constant. In [14], he proved a bound of the form

$$
\frac{|\chi(g)|}{\chi(1)} \leqslant \chi(1)^{-\gamma / n},
$$

when $G(q)$ is a classical group with natural module $V=\mathbb{F}_{q}^{n}$ of dimension $n$, and $\gamma=\gamma(q, d)$ is a positive real number depending on $q$ and on $d=\operatorname{dim}[V, g]$, the dimension of the commutator space of $g$ on $V$. While this result provides an exponential character bound $|\chi(g)| \leqslant \chi(1)^{\alpha_{g}}$, the exponent $\alpha_{g}=1-\gamma / n$ is not explicit, and in the general case we have $\gamma(q, d) \leqslant 0.001$, so $\alpha_{g} \geqslant 1-1 / 1000 n$, which is very close to 1 .

An explicit character bound for finite classical groups, with natural module $V=\mathbb{F}_{q}^{n}$, in terms of the support $\operatorname{supp}(g)$ of the element $g$ was obtained in [26, Theorem 4.3.6]. Namely,

$$
\frac{|\chi(g)|}{\chi(1)}<q^{-\sqrt{\operatorname{supp}(g)} / 481},
$$

where $\operatorname{supp}(g)$ is the codimension of the largest eigenspace of $g$ on $V \otimes_{\mathbb{F}_{q}} \overline{\mathbb{F}}_{q}$. These results have applications to covering numbers, mixing times and word maps.

In this paper we obtain asymptotically much stronger bounds for character ratios of finite groups of Lie type in good characteristic (this restriction comes from the fact that our proof relies on certain results in the Deligne-Lusztig theory, which currently are only known to hold in good characteristic). In fact, we provide the first explicit exponential 
character bounds for groups of Lie type, and show that these bounds are asymptotically optimal in many cases.

These character bounds lead to several new results on random walks and covering by products of conjugacy classes that are far stronger than previously known such results. Further applications to the theory of representation varieties of Fuchsian groups and probabilistic generation of groups of Lie type will be given in a sequel to this paper [37].

We also prove the first bounds on character ratios for Brauer characters, for the groups $\mathrm{SL}_{n}(q)$ and $\mathrm{GL}_{n}(q)$, and in characteristics coprime to $q$.

We now describe our results. Throughout the paper, let $\mathbb{K}$ be an algebraically closed field of characteristic $p, \mathcal{G}$ be a connected reductive algebraic group over $\mathbb{K}, F: \mathcal{G} \rightarrow \mathcal{G}$ be a Frobenius endomorphism, and $G=\mathcal{G}^{F}$. For a subgroup $X$ of $\mathcal{G}$ write $X_{\text {unip }}$ for the set of non-identity unipotent elements of $X$. For a fixed $F$, a Levi subgroup $\mathcal{L}$ of $\mathcal{G}$ will be called split, if it is an $F$-stable Levi subgroup of an $F$-stable parabolic subgroup of $\mathcal{G}$. For an $F$-stable Levi subgroup $\mathcal{L}$ of $\mathcal{G}$ and $L=\mathcal{L}^{F}$, we define

$$
\alpha(L):=\max _{u \in L_{\text {unip }}} \frac{\operatorname{dim} u^{\mathcal{L}}}{\operatorname{dim} u^{\mathcal{G}}} \quad \text { and } \quad \alpha(\mathcal{L}):=\max _{u \in \mathcal{L}_{\text {unip }}} \frac{\operatorname{dim} u^{\mathcal{L}}}{\operatorname{dim} u^{\mathcal{G}}}
$$

if $\mathcal{L}$ is not a torus, and $\alpha(L):=0$ and $\alpha(\mathcal{L}):=0$ otherwise.

TheOREM 1.1. There exists a function $f: \mathbb{N} \rightarrow \mathbb{N}$ such that the following statement holds. Let $\mathcal{G}$ be a connected reductive algebraic group such that $[\mathcal{G}, \mathcal{G}]$ is simple of rank $r$ over a field of good characteristic $p>0$. Let $G:=\mathcal{G}^{F}$ for a Frobenius endomorphism $F: \mathcal{G} \rightarrow \mathcal{G}$. Let $g \in G$ be any element such that $\mathbf{C}_{G}(g) \leqslant L:=\mathcal{L}^{F}$, where $\mathcal{L}$ is a proper split Levi subgroup of $\mathcal{G}$. Then, for any character $\chi \in \operatorname{Irr}(G)$ and $\alpha:=\alpha(L)$, we have

$$
|\chi(g)| \leqslant f(r) \chi(1)^{\alpha} .
$$

Remark 1.2. (i) The $\alpha$-bound in Theorem 1.1 is sharp in several cases - see Example 2.8. In fact, this $\alpha$-bound is always sharp in the case of $\mathrm{GL}_{n}(q)$ and $\mathrm{SL}_{n}(q)$, by Theorem 1.3.

(ii) If $r \geqslant 9$ and $q \geqslant r^{2}+1$, then the function $f(r)$ in Theorem 1.1 can be chosen to be $2^{2 r+\sqrt{2 r}+3}(r !)^{2}$ (with the main term being the square of the largest order of the Weyl group of a simple algebraic group of rank $r$ ) - see Proposition 2.7. Moreover, $\alpha \lesssim r$ $1-1 / r$ by Theorem 1.6 and $\chi(1) \geqslant \frac{1}{3} q^{r}$ if $\chi(1)>1$ by [24], and hence Theorem 1.1 yields $|\chi(g)|{ }_{r} \chi(1)^{\alpha+1 / 2 r} \lesssim_{r} \chi(1)^{1-1 / 2 r}$ if $q>r^{4 r}$. In fact, $\chi(1) \geqslant q^{r^{2} / 2}$ for most of $\chi \in \operatorname{Irr}(G)$, for which the bound becomes $|\chi(g)| \lesssim_{r} \chi(1)^{\alpha+1 / 3 r} \lesssim_{r} \chi(1)^{1-2 / 3 r}$ if $q>r^{12}$. (Here, we say that $f_{1}(x) \lesssim x f_{2}(x)$, for two functions $f_{1}, f_{2}: \mathbb{R} \rightarrow \mathbb{R}_{\geqslant 0}$, if $\lim \sup _{x \rightarrow \infty} f_{1}(x) / f_{2}(x) \leqslant 1$.)

(iii) Although the aforementioned choice of $f(r)$ in Theorem 1.1 can be improved, Example 2.8 (vi) shows that $f(r)$ should be at least the largest degree of complex irreducible characters of the Weyl group $W(\mathcal{G})$ of $\mathcal{G}$, which can be quite close to $|W(\mathcal{G})|^{1 / 2}$. 
In particular, choosing $\mathcal{G}$ of type $A_{r}$ and applying [38] and [53], we get

$$
f(r)>e^{-1.283 \sqrt{r+1}} \sqrt{(r+1) !} .
$$

Note that Theorem 1.1 and its various consequences also apply for finite twisted groups of Lie type (in good characteristic). The next result shows that the exponent $\alpha$ in Theorem 1.1 is optimal.

THEOREM 1.3. In the notation of Theorem 1.1, there is a constant $C_{n}>0$ depending only on $n$ such that the following statement holds. For $G=\mathcal{G}^{F}=\mathrm{GL}_{n}(q)$ with $q \geqslant C_{n}$ and for any proper split Levi subgroup $\mathcal{L}$ of $\mathcal{G}$, there is a semisimple element $g \in G$ and a unipotent character $\chi \in \operatorname{Irr}(G)$ such that $\mathbf{C}_{G}(g)=L=\mathcal{L}^{F}$ and

$$
|\chi(g)| \geqslant \frac{1}{4} \chi(1)^{\alpha(L)} \text {. }
$$

The same conclusion holds for $\mathrm{SL}_{n}(q)$, if for instance we choose $q$ so that $q-1$ is also divisible by $(n !)^{n}$.

In the case of $\mathrm{GL}_{n}(q)$ and $\mathrm{SL}_{n}(q)$ we can also prove a version of Theorem 1.1 for Brauer characters in cross-characteristic.

THEOREM 1.4. There exists a function $h: \mathbb{N} \rightarrow \mathbb{N}$ such that the following statement holds. Let $\mathcal{G}=\mathrm{GL}_{n}(\mathbb{K})$ or $\mathcal{G}=\mathrm{SL}_{n}(\mathbb{K})$ be an algebraic group over a field of characteristic $p>0$, and $F: \mathcal{G} \rightarrow \mathcal{G}$ be a Frobenius endomorphism such that $G=\mathcal{G}^{F} \cong \mathrm{GL}_{n}(q)$ or $\operatorname{SL}_{n}(q)$. Let $\ell=0$ or a prime not dividing $q$. Let $g \in G$ be any $\ell^{\prime}$-element such that $\mathbf{C}_{G}(g) \leqslant L:=\mathcal{L}^{F}$, where $\mathcal{L}$ is a proper split Levi subgroup of $\mathcal{G}$. Then, for any irreducible $\ell$-Brauer character $\varphi$ of $G$ and $\alpha:=\alpha(L)$, we have

$$
|\varphi(g)| \leqslant h(n) \varphi(1)^{\alpha} .
$$

The above results do not cover, for instance, the case where $g \in \mathcal{G}^{F}$ is a unipotent element. In fact, if $G=\mathrm{GL}_{n}(q)$ or $G=\mathrm{SL}_{n}(q)$, then Theorem 1.1 leaves out precisely those elements $g$ with semisimple part $s$ such that $\mathbf{C}_{\mathrm{GL}_{n}(q)}(s) \cong \mathrm{GL}_{n / a}\left(q^{a}\right)$, an extension-field subgroup. In other classical groups, there are further elements left out by Theorem 1.1, including those whose semisimple parts $s$ are quasi-isolated (i.e. $\mathbf{C}_{\mathcal{G}}(s)$ is not contained in any proper Levi subgroup of $\mathcal{G}$ ). However, we have been able to obtain a complete result covering all elements in $\mathrm{GL}_{n}(q)$ and $\mathrm{SL}_{n}(q)$.

THEOREM 1.5. There is a function $h: \mathbb{N} \rightarrow \mathbb{N}$ such that the following statement holds. For any $n \geqslant 5$, any prime power $q$, any irreducible complex character $\chi$ of $H=\mathrm{GL}_{n}(q)$ or $H=\mathrm{SL}_{n}(q)$, and any non-central element $g \in H$,

$$
|\chi(g)| \leqslant h(n) \chi(1)^{1-1 / 2 n} .
$$


For the remaining groups of Lie type, character bounds of a somewhat different kind, which work for arbitrary elements $g \in \mathcal{G}^{F}$, and are weaker than the one in Theorem 1.1, but asymptotically stronger than the ones in [14] and [26], will be proved in a sequel to this paper. We also note [16], which explores a completely different approach and establishes exponential character bounds for $|\chi(g)|$, where either $g$ is an element of a finite group of Lie type $G$ with not-too-large centralizer $\mathbf{C}_{G}(g)$ or $\chi \in \operatorname{Irr}(G)$ has not-toolarge degree.

To be able to apply Theorem 1.1, we need information on the values of $\alpha(L) \leqslant \alpha(\mathcal{L})$. For classical groups, we prove the following upper bound.

THEOREM 1.6. If $\mathcal{G}$ is a classical algebraic group over $\mathbb{K}$ in good characteristic, and $\mathcal{L}$ is a Levi subgroup of $\mathcal{G}$, then

$$
\alpha(\mathcal{L}) \leqslant \frac{1}{2}\left(1+\frac{\operatorname{dim} \mathcal{L}}{\operatorname{dim} \mathcal{G}}\right) .
$$

For exceptional types we obtain fairly complete information.

THEOREM 1.7. If $\mathcal{G}$ is an exceptional algebraic group in good characteristic, the values of $\alpha(\mathcal{L})$ for (proper, non-toral) Levi subgroups $\mathcal{L}$ are as in Table 1.

In Table 1 , for $\mathcal{G}=F_{4}$ or $\mathcal{G}=G_{2}$ the symbols $\tilde{A}_{1}$ and $\tilde{A}_{2}$ refer to Levi subsystems consisting of short roots. For $\mathcal{G}=E_{7}$, there are two Levi subgroups $A_{5}$ and $A_{5}^{\prime}$ : using the notation for the fundamental roots $\alpha_{i}, 1 \leqslant i \leqslant 7$, as in [3], these are the Levi subgroups with fundamental roots $\left\{\alpha_{i}: i=1,3,4,5,6\right\}$ and $\left\{\alpha_{i}: i=2,4,5,6,7\right\}$, respectively. The notation $\triangleright A_{4}$, for instance, means that $\mathcal{L}^{\prime}=[\mathcal{L}, \mathcal{L}]$ has a simple factor of type $A_{4}$. Furthermore, in this table and elsewhere, we will also sometimes denote a simple algebraic group by the type of its Dynkin diagram, like $A_{4}, C_{3}$, etc., and use $T_{m}$ to denote an $m$-dimensional torus.

We can now easily deduce the following corollary.

Corollary 1.8. Let $\mathcal{G}, G=\mathcal{G}^{F}$, and $f$ be as in Theorem 1.1. Suppose that $y \in G$ is a (semisimple) element such that $\mathbf{C}_{G}(y)=\mathcal{L}^{F}$, where $\mathcal{L}$ is a proper split Levi subgroup of $\mathcal{G}$. Then, for any non-linear $\chi \in \operatorname{Irr}(G)$,

$$
|\chi(y)| \leqslant f(r) \chi(1)^{1-\operatorname{dim} y^{\mathcal{G}} / 2 \operatorname{dim} \mathcal{G}}
$$

Next, we establish a new bound on character ratios of certain elements, in terms of the support (which is defined right after (1.2)) of its semisimple part. 


\begin{tabular}{|c|c|c|c|c|c|c|c|c|c|c|c|}
\hline$E_{8}$ & $\begin{array}{c}\mathcal{L}^{\prime} \\
\alpha(\mathcal{L})\end{array}$ & $\begin{array}{l}E_{7} \\
\frac{17}{29}\end{array}$ & $\begin{array}{c}D_{7} \\
\frac{9}{23}\end{array}$ & $\begin{array}{c}\mathcal{L}^{\prime} \triangleright E_{6} \\
\frac{11}{29}\end{array}$ & $\begin{array}{l}D_{6} \\
\frac{9}{29}\end{array}$ & $\begin{array}{l}A_{7} \\
\frac{15}{56}\end{array}$ & $\begin{array}{c}\triangleright D_{5} \\
\frac{7}{29}\end{array}$ & $\begin{array}{c}\triangleright A_{6} \\
\frac{5}{23}\end{array}$ & $\begin{array}{c}\triangleright A_{5} \\
\frac{4}{23}\end{array}$ & $\begin{array}{c}\triangleright D_{4} \\
\frac{5}{29}\end{array}$ & $\begin{array}{l}\text { rest } \\
\leqslant \frac{1}{6}\end{array}$ \\
\hline$E_{7}$ & $\begin{array}{c}\mathcal{L}^{\prime} \\
\alpha(\mathcal{L})\end{array}$ & $\begin{array}{l}E_{6} \\
\frac{11}{17}\end{array}$ & $\begin{array}{c}D_{6} \\
\frac{5}{9}\end{array}$ & $\begin{array}{c}\mathcal{L}^{\prime} \triangleright D_{5} \\
\frac{7}{17}\end{array}$ & $\begin{array}{l}A_{6} \\
\frac{5}{13}\end{array}$ & $\begin{array}{l}A_{5} \\
\frac{4}{13}\end{array}$ & $\begin{array}{c}\triangleright A_{5}^{\prime} \\
\frac{1}{3}\end{array}$ & $\begin{array}{c}\triangleright D_{4} \\
\frac{5}{17}\end{array}$ & $\begin{array}{l}\triangleright A_{4} \\
\leqslant \frac{1}{4}\end{array}$ & $\begin{array}{l}\triangleright A_{3} \\
\leqslant \frac{1}{5}\end{array}$ & $\begin{array}{l}\text { rest } \\
\leqslant \frac{1}{6}\end{array}$ \\
\hline$E_{6}$ & $\begin{array}{c}\mathcal{L}^{\prime} \\
\alpha(\mathcal{L})\end{array}$ & $\begin{array}{l}D_{5} \\
\frac{7}{11}\end{array}$ & $\begin{array}{c}A_{5} \\
\frac{1}{2}\end{array}$ & $\begin{array}{l}D_{4} \\
\frac{5}{11}\end{array}$ & $\begin{array}{c}\mathcal{L}^{\prime} \triangleright A_{4} \\
\frac{3}{8}\end{array}$ & $\begin{array}{c}\triangleright A_{3} \\
\frac{3}{11}\end{array}$ & $\begin{array}{l}\triangleright A_{2} \\
\leqslant \frac{7}{27}\end{array}$ & $\begin{aligned} & A_{1}^{k} \\
\leqslant & \frac{3}{20}\end{aligned}$ & & & \\
\hline$F_{4}$ & $\begin{array}{c}\mathcal{L}^{\prime} \\
\alpha(\mathcal{L})\end{array}$ & $\begin{array}{c}B_{3} \\
\frac{1}{2}\end{array}$ & $\begin{array}{l}C_{3} \\
\frac{7}{15}\end{array}$ & $\begin{array}{c}A_{2} \tilde{A}_{1}, A_{2} \\
\frac{1}{4}\end{array}$ & $\begin{array}{c}\tilde{A}_{2} A_{1} \\
\frac{2}{9}\end{array}$ & $\begin{array}{c}\tilde{A}_{2} \\
\frac{1}{5}\end{array}$ & $\begin{array}{c}A_{1} \tilde{A}_{1} \\
\frac{1}{7}\end{array}$ & $\begin{array}{c}A_{1} \\
\frac{1}{8}\end{array}$ & $\begin{array}{l}\tilde{A}_{1} \\
\frac{1}{11}\end{array}$ & & \\
\hline$G_{2}$ & $\begin{array}{c}\mathcal{L}^{\prime} \\
\alpha(\mathcal{L})\end{array}$ & $\begin{array}{c}A_{1} \\
\frac{1}{3}\end{array}$ & $\begin{array}{c}\tilde{A}_{1} \\
\frac{1}{4}\end{array}$ & & & & & & & & \\
\hline
\end{tabular}

Table 1. $\alpha$-values for exceptional groups.

THEOREM 1.9. Assume $\mathcal{G}=\mathrm{SL}_{n}(\mathbb{K})$ with $n \geqslant 2, \mathcal{G}=\operatorname{Sp}_{n}(\mathbb{K})$ with $n \geqslant 4$, or $\mathcal{G}=\operatorname{Spin}_{n}(\mathbb{K})$ with $n \geqslant 7$, all in good characteristic, and define

$$
c:=c(\mathcal{G})= \begin{cases}\frac{r+1}{2 r+4}, & \text { if } \mathcal{G}=\mathrm{SL}_{r+1}, \\ \frac{r}{4 r+2}, & \text { if } \mathcal{G}=\mathrm{Sp}_{2 r}, \\ \frac{r}{4 r-2}, & \text { if } \mathcal{G}=\mathrm{Spin}_{2 r}, \\ \frac{1}{4}, & \text { if } \mathcal{G}=\operatorname{Spin}_{2 r+1} .\end{cases}
$$

Let $G=\mathcal{G}^{F}=G(q)$ be defined over $\mathbb{F}_{q}$ and $f$ be as in Theorem 1.1, and let $g \in G$ be any element such that its semisimple part y has centralizer $\mathbf{C}_{G}(y)=\mathcal{L}^{F}$, where $\mathcal{L}$ is a proper split Levi subgroup of $\mathcal{G}$. Then, for any non-linear $\chi \in \operatorname{Irr}(G)$,

$$
\frac{|\chi(g)|}{\chi(1)} \leqslant 3 f(r) q^{-c \operatorname{supp}(y)} \text {. }
$$

In particular, it follows that $|\chi(y)| / \chi(1) \leqslant 3 f(r) \chi(1)^{-c \operatorname{supp}(y)}$, and that, for any $\varepsilon>0$, $r \geqslant r(\varepsilon)$ and $q$ larger than a suitable function of $r$, we have

$$
\frac{|\chi(y)|}{\chi(1)} \leqslant q^{-(b-\varepsilon) \operatorname{supp}(y)},
$$

where $b=\frac{1}{2}$ in the $\mathrm{SL}_{r+1}$ case and $b=\frac{1}{4}$ in the other cases. 
Theorem 1.9 and its consequences considerably improve the bound (1.2) from [26, Theorem 4.3.6] for elements as above.

We also obtain more precise character bounds for $\mathrm{GL}_{n}(q)$. To state them we need some notation. For positive integers $n_{1}, \ldots, n_{m}$, with $\max _{1 \leqslant i \leqslant m} n_{i} \geqslant 2$, define

$$
\beta\left(n_{1}, \ldots, n_{m}\right):=\max _{a_{i j}} \frac{\sum_{i=1}^{m}\left(n_{i}^{2}-\sum_{j=1}^{n} a_{i j}^{2}\right)}{n^{2}-\sum_{j=1}^{n}\left(\sum_{i=1}^{m} a_{i j}\right)^{2}},
$$

where $n=n_{1}+\ldots+n_{m}$ and the maximum is taken over all non-negative integers $a_{i j}$, with $1 \leqslant i \leqslant m$ and $1 \leqslant j \leqslant n$, satisfying

$$
\sum_{j=1}^{n} a_{i j}=n_{i}, \quad a_{i 1} \geqslant a_{i 2} \geqslant \ldots \geqslant a_{i n}, \quad 1 \leqslant i \leqslant m, \quad \text { and } \quad \max _{1 \leqslant i \leqslant m} a_{i 2}>0 .
$$

We also let $\beta(1,1, \ldots, 1)=0$.

TheOrem 1.10. Let $G=\mathrm{GL}_{n}(q)$ and let $L \leqslant G$ be a Levi subgroup of the form $L=$ $\mathrm{GL}_{n_{1}}(q) \times \ldots \times \mathrm{GL}_{n_{m}}(q)$, where $n_{i} \geqslant 1$ and $\sum_{i=1}^{m} n_{i}=n$. Let $n_{i_{0}}=\max _{1 \leqslant i \leqslant m} n_{i}$. Then,

$$
\frac{n_{i_{0}}-1}{n-t} \leqslant \alpha(L)=\beta\left(n_{1}, \ldots, n_{m}\right) \leqslant \frac{n_{i_{0}}}{n}
$$

if $n_{i_{0}} \geqslant 2$ and $t$ is the number of $1 \leqslant j \leqslant m$ such that $n_{j}=n_{i_{0}}$, and

$$
\alpha(L)=\beta\left(n_{1}, \ldots, n_{m}\right)=0
$$

if $n_{i_{0}}=1$. Consequently, for every $g \in G$ with $\mathbf{C}_{G}(g) \leqslant L$ and every $\chi \in \operatorname{Irr}(G)$, we have

$$
|\chi(g)| \leqslant f(n-1) \chi(1)^{\beta\left(n_{1}, \ldots, n_{m}\right)},
$$

where $f: \mathbb{N} \rightarrow \mathbb{N}$ is the function specified in Theorem 1.1.

Suppose now that $m$ divides $n$ and $n_{1}=\ldots=n_{m}=n / m>1$. Then, we can show that $\beta\left(n_{1}, \ldots, n_{m}\right)=1 / m$, so we immediately obtain the following.

Corollary 1.11. Let $G=\operatorname{GL}_{n}(q)$, where $q$ is a prime power. Let $m<n$ be a divisor of $n$ and $L \leqslant G$ be a Levi subgroup of the form $L=\mathrm{GL}_{n / m}(q)^{m}$. Let $g \in G$ with $\mathbf{C}_{G}(g) \leqslant L$. Then, we have

$$
|\chi(g)| \leqslant f(n-1) \chi(1)^{1 / m}
$$

for all characters $\chi \in \operatorname{Irr}(G)$, where $f: \mathbb{N} \rightarrow \mathbb{N}$ is the function specified in Theorem 1.1. 
Example 2.8 again shows that the exponent $1 / m$ in Corollary 1.11 is sharp. In general, Theorem 1.10 determines $\alpha(L)$ up to within $1 / n$. It is reasonable to conjecture that, under the hypotheses of Theorem $1.10, \alpha(L)=\left(n_{i_{0}}-1\right) /(n-t)$. This conjecture is confirmed in Theorem 4.13 for the case $m=2$ (as well as in the cases where either $n \leqslant 8$, or $m \leqslant 4$ and $n \leqslant 13$, by direct calculation).

The bound in Theorem 1.10 and some variations on it have applications to Fuchsian groups (see [37]). Corollary 1.11 may be regarded as a Lie analogue of the Fomin-Lulov character bound (1.1) for $\mathrm{S}_{n}$ mentioned before.

We now present some applications of the above results to the theory of mixing times for random walks on finite quasisimple groups of Lie type corresponding to conjugacy classes. Let $G=G(q)$ be such a group, let $y \in G$ be a non-central element, and let $C=y^{G}$, the conjugacy class of $y$. Consider the random walk on the corresponding Cayley graph starting at the identity, and at each step moving from a vertex $g$ to a neighbour $g s$, where $s \in y^{G}$ is chosen uniformly at random. Let $P^{t}(g)$ be the probability of reaching the vertex $g$ after $t$ steps. The mixing time of this random walk is defined to be the smallest integer $t=T(G, y)$ such that $\left\|P^{t}-U\right\|_{1}<1 / e$, where $U$ is the uniform distribution and $\|f\|_{1}=\sum_{g \in G}|f(g)|$ is the $l_{1}$-norm.

Mixing times of such random walks have been extensively studied since the pioneering work of Diaconis and Shashahani [6] on the case $G=\mathrm{S}_{n}$, with $C$ being the class of transpositions in $S_{n}$. Additional results on random walks in symmetric and alternating groups have been obtained in various papers; see for instance [43], [54], [40] and [25]. The latter paper obtains essentially optimal results on mixing times in these groups.

However, if we turn from symmetric groups to finite groups $G$ of Lie type, good estimates on mixing times have been obtained only in very few cases. Hildebrand [17] showed that the mixing time for the class of tranvections in $\mathrm{SL}_{n}(q)$ is of the order of $n$. In [36] it is shown that if $y \in G$ is a regular element, then the mixing time $T(G, y)$ is 2 when $G \neq \mathrm{PSL}_{2}(q)$ is large. In [44] it is proved that, if $G$ is any finite simple group, then, for a random $y \in G$, we have $T(G, y)=2$ (namely, the latter equality holds with probability tending to 1 as $|G| \rightarrow \infty)$. Other than that, the mixing times $T(G, y)$ for groups $G$ of Lie type remain a mystery.

The next result contains bounds for mixing times, and also (in parts (I) (a) and (II)) for the number of steps required so that $P^{t}$ is close to $U$ in the $l_{\infty}$-norm, which is stronger than the $l_{1}$-norm condition for mixing time (and also implies that the random walks hits all elements of $G$ ). Here, we define $\|f\|_{\infty}=|G| \max _{x \in G}|f(x)|$, and say that $C^{t}=G$ almost uniformly pointwise as $q \rightarrow \infty$ if $\left\|P^{t}-U\right\|_{\infty} \rightarrow 0$ as $q \rightarrow \infty$. 
We denote by $h:=h(\mathcal{G})$ the Coxeter number of $\mathcal{G}$, defined by

$$
h(\mathcal{G})=\frac{\operatorname{dim} \mathcal{G}}{r}-1
$$

where $r$ is the rank of $\mathcal{G}$. Note that $h \geqslant 2$ and that $h \rightarrow \infty$ as $r \rightarrow \infty$.

THEOREM 1.12. Let $\mathcal{G}$ be a simple algebraic group in good characteristic, and $G=$ $G(q)=\mathcal{G}^{F}$ be a finite quasisimple group over $\mathbb{F}_{q}$. Let $y \in G$ be such that $\mathbf{C}_{G}(y) \leqslant L$, where $L=\mathcal{L}^{F}$ for a proper split Levi subgroup $\mathcal{L}$ of $\mathcal{G}$. Write $C=y^{G}$.

(I) Suppose $\mathcal{G}$ is of classical type.

(a) If

$$
t>\left(4+\frac{4}{h}\right) \frac{\operatorname{dim} \mathcal{G}}{\operatorname{dim} \mathcal{G}-\operatorname{dim} \mathcal{L}},
$$

then $C^{t}=G$ almost uniformly pointwise as $q \rightarrow \infty$. In particular, $C^{t}=G$ for sufficiently large $q$.

(b) The mixing time $T(G, y)$ satisfies

$$
T(G, y) \leqslant\left\lceil\left(2+\frac{2}{h}\right) \frac{\operatorname{dim} \mathcal{G}}{\operatorname{dim} \mathcal{G}-\operatorname{dim} \mathcal{L}}\right\rceil
$$

for large $q$.

(II) Suppose $\mathcal{G}$ is of exceptional type. Then, $C^{6}=G$ almost uniformly pointwise as $q \rightarrow \infty$, and $T(G, y) \leqslant 3$.

Remarks. (i) Note that the multiplicative constants above are very small. For example, $2+2 / h \leqslant 3$ and it tends to 2 as $r \rightarrow \infty$.

(ii) The constant $2+2 / h$ in part (I) (b) of Theorem 1.12 is best possible for some classes, for example homologies $y=\operatorname{diag}\left(\mu I_{n-1}, \lambda\right)$ in $G=\mathrm{SL}_{n}(q)$ (where $\mu, \lambda \in \mathbb{F}_{q}^{\times}$and $\mu \neq \lambda$, and so $q \geqslant 3$ ), for which the bound given by part (I) (b) is $T(G, y) \leqslant n+3$ and for which the mixing time is at least $n$ by Lemma 5.2 (ii).

(iii) The bound $T(G, y) \leqslant 3$ for exceptional groups in (II) is best possible for those conjugacy classes $y^{G}$ with $\mathbf{C}_{G}(y)$ contained in a proper split Levi subgroup $L$ and $\operatorname{dim} y^{\mathcal{G}}<\frac{1}{2} \operatorname{dim} \mathcal{G}$. For such classes, $\left|y^{G}\right|^{2}<\frac{1}{2}|G|$ for large $q$, so the mixing time cannot be 2 , by Lemma 5.2 (i).

Theorem 1.12 (I) (b) implies the following linear bounds for classical groups.

Corollary 1.13. Let $G=\mathcal{G}^{F}$ be a quasisimple classical group over $\mathbb{F}_{q}$, where $\mathcal{G}$ is simple of rank $r$ over $\mathbb{F}_{q}$ in good characteristic, and let $y \in G$ be as in Theorem 1.12. Then, for large $q$, the following properties hold:

(i) $\operatorname{diam}\left(G, y^{G}\right) \leqslant 2 r+4$;

(ii) $T(G, y) \leqslant r+2$. 
A linear bound for the diameter (of the order of 40r), which holds for all non-central conjugacy classes, can be found in [27].

Using Theorem 1.5, we can obtain such a bound for all conjugacy classes in $\mathrm{SL}_{n}(q)$.

Corollary 1.14. Let $G=\operatorname{SL}_{n}(q)$, let $x$ be an arbitrary non-central element of $G$ and let $C=x^{G}$.

(i) If $t>4 n+4$, then $C^{t}=G$ almost uniformly pointwise as $q \rightarrow \infty$.

(ii) The mixing time satisfies $T(G, x) \leqslant 2 n+3$ for large $q$.

Note that $\left[28\right.$, Theorem 1] shows that $C^{n}=G$ for any non-trivial conjugacy class $C$ of $G=\operatorname{PSL}_{n}(q)$, where $n \geqslant 3$ and $q \geqslant 4$.

We can also use Theorem 3.3 (or rather its corollary 3.5) to obtain a better bound for unipotent elements of $\mathrm{SL}_{n}(q)$.

THEOREM 1.15. Let $G=\mathrm{SL}_{n}(q)$ and let $u$ be a non-identity unipotent element in $G$. Write $C=u^{G}$.

(i) If $t>2 n$, then $C^{t}=G$ almost uniformly pointwise as $q \rightarrow \infty$. In particular, $C^{t}=G$ for sufficiently large $q$.

(ii) The mixing time satisfies $T(G, u) \leqslant n$ for sufficiently large $q$.

One can compare part (ii) of the above theorem with Hildebrand's result [17] for transvections, where he proves that for $n$ varying, the mixing time for the class of tranvections in $\mathrm{SL}_{n}(q)$ is of the order of $n$. In our case $n$ may still vary, but $q$ should be much larger than $n$. The coincidence of values seems striking.

It is interesting to compare the mixing time $T(G, y)$ with the covering number $\operatorname{cn}(G, C)$ of the conjugacy class $C=y^{G}$, defined as the minimal $t$ for which $C^{t}=G$. It is known that there is an absolute constant $b$ such that for any conjugacy class $C \neq\{1\}$ of any finite simple group $G$ we have

$$
\frac{\log |G|}{\log |C|} \leqslant c n(G, C) \leqslant b \frac{\log |G|}{\log |C|} .
$$

Indeed, the first inequality is trivial, while the second is [34, Corollary 1.2].

It is easy to see that, with the above notation,

$$
\frac{\log |G|+\log \left(1-e^{-1}\right)}{\log |C|} \leqslant T(G, y) .
$$

Indeed, this follows from Lemma 5.2.

It is conjectured in [46, Conjecture 4.3] that there is an absolute constant $c$ such that, for any finite simple group $G$ of Lie type and any non-identity element $y \in G$, we have

$$
T(G, y) \leqslant c \frac{\log |G|}{\log |C|},
$$

where $C=y^{G}$. 
Note that this statement does not hold for alternating groups $G$ (take $y \in G$ to be a cycle of length around $\frac{1}{2} n$ - then $\log |G| / \log |C|$ is bounded, while $T(G, y)$ is of the order of $\log n)$.

The above conjecture is related to an older conjecture posed by Lubotzky in [39, p. 179]. Lubotzky conjectured that, if $G$ is a finite simple group and $C$ is a non-trivial conjugacy class of $G$, then the mixing time of the Cayley graph $\Gamma(G, C)$ of $G$ with $C$ as a generating set is linearly bounded above in terms of the diameter of $\Gamma(G, C)$. As observed above, this conjecture is false for alternating groups; however, it remains open for groups of Lie type. Since this diameter is exactly the covering number $c n(G, C)$, this conjecture (combined with the more recent upper bound on $\operatorname{cn}(G, C)$ mentioned above) implies conjecture (1.4).

Applying Theorem 1.12, we are able to prove the above conjectures in some interesting cases.

COROLlaRY 1.16. Let $\mathcal{G}$ be a simple algebraic group in good characteristic, and $G=G(q)=\mathcal{G}^{F}$ a finite quasisimple group over $\mathbb{F}_{q}$. Suppose $q$ is large (given $\left.\mathcal{G}\right)$. Then, conjecture (1.4) holds for all non-central elements $y \in G$, whenever $\mathbf{C}_{G}(y)=\mathcal{L}^{F}$ for a proper split Levi subgroup $\mathcal{L}$ of $\mathcal{G}$.

Indeed, this readily follows from part I (b) of Theorem 1.12, with a very small constant $c$ (around 3).

Conjecture (1.4) and Corollary 1.16 suggest a distinctive difference between mixing times for $\mathrm{S}_{n}$ as opposed to classical groups $\mathrm{Cl}_{n}(q)$.

Our final result essentially determines the mixing time $T(G, y)$ in terms of the support of $y$ as follows (recall the notation $f_{1}(x) \lesssim x f_{2}(x)$ from Remark 1.2).

THEOREM 1.17. Let $\mathcal{G}$ be a simple, simply connected, classical algebraic group of rank $r \geqslant 1$ over $\mathbb{K}$ of good characteristic $p>0$. Let $F: \mathcal{G} \rightarrow \mathcal{G}$ be a Frobenius endomorphism such that $G:=\mathcal{G}^{F}$ is defined over $\mathbb{F}_{q}$, where $q$ is large enough in comparison to $r$. Also, define

$$
r^{\prime}:=r^{\prime}(\mathcal{G})= \begin{cases}\frac{r(2 r+4)}{r+1}, & \text { if } \mathcal{G}=\mathrm{SL}_{r+1}, \\ 4 r+2, & \text { if } \mathcal{G}=\mathrm{Sp}_{2 r}, r \geqslant 2, \\ 4 r-2, & \text { if } \mathcal{G}=\mathrm{Spin}_{2 r}, r \geqslant 4, \\ 4 r, & \text { if } \mathcal{G}=\mathrm{Spin}_{2 r+1}, r \geqslant 3 .\end{cases}
$$

Let $g \in G$ be any element such that its semisimple part $y$ has centralizer $\mathbf{C}_{G}(y)=\mathcal{L}^{F}$, where $\mathcal{L}$ is a proper split Levi subgroup of $\mathcal{G}$. Then, we have

$$
T(G, g) \leqslant\left\lceil\left(2+\frac{2}{h}\right) \frac{r^{\prime}}{\operatorname{supp}(y)}\right\rceil .
$$


Furthermore, we have

$$
\frac{1}{2} \frac{r^{\prime}}{\operatorname{supp}(y)} \lesssim|G| T(G, y) \leqslant\left\lceil\left(2+\frac{2}{h}\right) \frac{r^{\prime}}{\operatorname{supp}(y)}\right\rceil .
$$

Thus, under the above conditions, the mixing time $T(G, y)$ is essentially $r / \operatorname{supp}(y)$ (up to a small multiplicative constant).

\section{Character bounds: Proof of Theorem 1.1}

Throughout this section, let $\mathcal{G}$ be a connected reductive algebraic group over a field of characteristic $p>0, F: \mathcal{G} \rightarrow \mathcal{G}$ be a Frobenius endomorphism, and let $G:=\mathcal{G}^{F}$. We will say that $\mathcal{G}^{F}$ is defined over $\mathbb{F}_{q}$ if $q$ is the common absolute value of the eigenvalues of $F$ acting on $X(\mathcal{T}) \otimes \mathbb{R}$, where $X(\mathcal{T})$ is the character group of an $F$-stable maximal torus $\mathcal{T}$ of $\mathcal{G}$.

First, we prove the following statement concerning Harish-Chandra restriction.

Proposition 2.1. Let $g \in G$ be such that $\mathbf{C}_{G}(g) \leqslant \mathcal{L}^{F}$, where $\mathcal{L}$ is an F-stable Levi subgroup of an $F$-stable parabolic subgroup $\mathcal{P}=\mathcal{U} \mathcal{L}$ of $\mathcal{G}$ with unipotent radical $\mathcal{U}$. Let $\ell=0$ or $\ell$ be a prime not dividing $p|g|, \mathbb{F}$ be an algebraically closed field of characteristic $\ell$, and let $\varphi$ be the Brauer character of some $\mathbb{F} G$-module $V$. Also, let $\psi$ denote the Brauer character of the $\mathcal{L}^{F}$-module $\mathbf{C}_{V}\left(\mathcal{U}^{F}\right)$. Then,

$$
\varphi(g)=\psi(g) .
$$

Proof. (a) Write $L:=\mathcal{L}^{F}, P:=\mathcal{P}^{F}$, and $U:=\mathcal{U}^{F}$. First, we handle the case $\ell=0$. Consider the map $f: U \rightarrow U$ given by $f(u)=g^{-1} u g u^{-1}$. Then, for $u, v \in U$, we have that

$$
f(u)=f(v) \quad \Longleftrightarrow v^{-1} u \in U \cap \mathbf{C}_{G}(g) \subseteq U \cap L=1 \quad \Longleftrightarrow u=v .
$$

Thus, the map $f$ is injective, and so bijective. Hence, when $u$ runs over $U, u g u^{-1}$ runs over the elements of $g U$, each element once:

$$
\left\{u g u^{-1}: u \in U\right\}=g U \text {. }
$$

Now, we decompose $V=\mathbf{C}_{V}(U) \oplus[V, U]$ as a $P$-module (note that $P=\mathbf{N}_{G}(U)$ ), and let $\Phi=\operatorname{diag}\left(\Phi_{1}, \Phi_{2}\right)$ denote the representation of $P$ with respect to some basis respecting this decomposition. In particular, no irreducible constituent of $\left.\left(\Phi_{2}\right)\right|_{U}$ is trivial, and so $\sum_{u \in U} \Phi_{2}(u)=0$. It follows that

$$
\begin{aligned}
\sum_{u \in U} \Phi\left(u g u^{-1}\right) & =\sum_{u \in U} \Phi(g u)=\Phi(g) \sum_{u \in U} \Phi(u) \\
& =\operatorname{diag}\left(\Phi_{1}(g) \sum_{u \in U} \Phi_{1}(u), \Phi_{2}(g) \sum_{u \in U} \Phi_{2}(u)\right)=\operatorname{diag}\left(|U| \Phi_{1}(g), 0\right) .
\end{aligned}
$$

Taking the trace of both sides, we obtain $|U| \varphi(g)=|U| \psi(g)$, as stated. 
(b) For the modular case $\ell>0$, let $\chi^{\circ}$ denote the restriction of any complex character $\chi$ of $G$ or $P$ to $\ell^{\prime}$-elements. It is well known, see e.g. [20, Theorem 15.14], that any Brauer character of $G$ is a $\mathbb{Z}$-combination of $\chi^{\circ}$ with $\chi \in \operatorname{Irr}(G)$. It follows that (in the Grothendieck group of $\mathbb{F} G$-modules) we can write $V=V_{1}-V_{2}$, where $V_{1}$ (resp. $V_{2}$ ) is some reduction modulo $\ell$ of the $\mathbb{C} G$-module $W_{1}$ (resp. $W_{2}$ ) affording the complex character $\chi_{1}$ (resp. $\chi_{2}$ ). Since $\ell \neq p, \mathbf{C}_{V}(U)=\mathbf{C}_{V_{1}}(U)-\mathbf{C}_{V_{2}}(U)$ in the Grothendieck group of $\mathbb{F} P$ modules. Now, $g \in P$ and $\varphi(g)=\chi_{1}(g)-\chi_{2}(g)$, so the statement follows by applying the results of (a) to $W_{1}$ and $W_{2}$.

Recall that the complex irreducible characters of $G=\mathcal{G}^{F}$ can be partitioned into Harish-Chandra series; see [5, Chapter 9]. We refer to [5] and [8] for basic facts on Harish-Chandra restriction ${ }^{*} R_{L}^{G}$ and Harish-Chandra induction $R_{L}^{G}$. We will also need the following fact (which is well known to the experts, but the proof of which is given for the reader's convenience).

Proposition 2.2. There is a constant $A=A(r)$ depending only on the semisimple rank $r$ of $\mathcal{G}$ with the following property. Suppose that $\chi \in \operatorname{Irr}(G)$ is such that ${ }^{*} R_{L}^{G}(\chi) \neq 0$ for $L=\mathcal{L}^{F}$, where $\mathcal{L}$ is a proper split Levi subgroup of $\mathcal{G}$. Then, the total number of irreducible constituents of the L-character ${ }^{*} R_{L}^{G}(\chi)$ (counting multiplicities) is at most $A$. In fact, if $[\mathcal{G}, \mathcal{G}]$ is simple, then one can choose $A=W(r)^{2}$, where $W(r)$ denotes the largest order of the Weyl group of a simple algebraic group of rank $r$.

Proof. Since ${ }^{*} R_{L}^{G}(\chi) \neq 0, \chi$ is not cuspidal. By [5, Proposition 9.3.1], we may assume that $\mathcal{L}$ is a standard $F$-stable Levi subgroup of a standard $F$-stable parabolic subgroup $\mathcal{P}=\mathcal{U} \mathcal{L}$ of $\mathcal{G}$. Suppose that $\chi$ belongs to the Harish-Chandra series labeled by a standard Levi subgroup $L_{1}$ and a cuspidal character $\psi \in \operatorname{Irr}\left(L_{1}\right)$. Here, $L_{1}=\mathcal{L}_{1}^{F}$, where $\mathcal{L}_{1}$ is a proper split Levi subgroup of $\mathcal{G}$, and $\chi$ is an irreducible constituent of $R_{L_{1}}^{G}(\psi)$.

Suppose now that $\eta$ is any irreducible constituent of ${ }^{*} R_{L}^{G}(\chi)$, and let $\eta$ belong to the Harish-Chandra series labeled by a standard Levi subgroup $L_{2}$ (of $L$ ) and a cuspidal character $\delta \in \operatorname{Irr}\left(L_{2}\right)$. Then, $\eta$ is an irreducible constituent of $R_{L_{2}}^{L}(\delta)$. Then, by the adjointness of the Harish-Chandra induction and restriction and their transitivity [8, Proposition 4.7], we have that

$$
\begin{aligned}
0 & <c_{\eta}:=\left[{ }^{*} R_{L}^{G}(\chi), \eta\right]_{L}=\left[\chi, R_{L}^{G}(\eta)\right]_{G} \\
& \leqslant\left[\chi, R_{L}^{G}\left(R_{L_{2}}^{L}(\delta)\right)\right]_{G}=\left[\chi, R_{L_{2}}^{G}(\delta)\right]_{G} \leqslant\left[R_{L_{1}}^{G}(\psi), R_{L_{2}}^{G}(\delta)\right]_{G} .
\end{aligned}
$$

Since $\psi \in \operatorname{Irr}\left(L_{1}\right)$ and $\delta \in \operatorname{Irr}\left(L_{2}\right)$ are cuspidal, it follows by [5, Proposition 9.1.5] that the pair $\left(L_{1}, \psi\right)$ is $G$-conjugate to the pair $\left(L_{2}, \delta\right)$ and $R_{L_{1}}^{G}(\psi)=R_{L_{2}}^{G}(\delta)$. Hence, with no loss of generality, we may replace $\left(L_{1}, \psi\right)$ by $\left(L_{2}, \delta\right)$. Furthermore, by [5, Proposition 9.2.4], $\left[R_{L_{1}}^{G}(\psi), R_{L_{1}}^{G}(\psi)\right]_{G}$ can be bounded by the order of the Weyl group $W(\mathcal{G})$ of $\mathcal{G}$, and so in 
terms of the semisimple rank $r$ as well. Thus we can bound $c_{\eta}$ in terms of $r$. The same is true for $\left[R_{L_{1}}^{L}(\psi), R_{L_{1}}^{L}(\psi)\right]_{L}$, and so for the number of possibilities for $\eta$. In particular, if $[\mathcal{G}, \mathcal{G}]$ is simple, then $|W(\mathcal{L})| \leqslant|W(\mathcal{G})| \leqslant W(r)$ and so we can choose $A(r)=W(r)^{2}$.

From now on, we assume that $p$ is a good prime for $\mathcal{G}$ (and $\mathbb{K}=\overline{\mathbb{K}}$ is a field of characteristic $p$ ). Then, a theory of generalized Gelfand-Graev representations (GGGRs) was developed by Kawanaka [22]: with each unipotent element $u \in G=\mathcal{G}^{F}$ one may associate a GGGR with character $\Gamma_{u}$ (which depends only the conjugacy class of $u$ in $G$ ).

Suppose now that $\mathcal{O}=u^{\mathcal{G}}$ is an $F$-stable unipotent conjugacy class in $\mathcal{G}$. By the Lang-Steinberg theorem, since $\mathcal{G}$ is connected, we may assume that $u \in G$. Then, $\mathcal{O}$ is called a unipotent support for a given $\varrho \in \operatorname{Irr}(G)$ if

(i) $\sum_{g \in \mathcal{O}^{F}} \varrho(g) \neq 0$;

(ii) If $\mathcal{O}^{\prime}$ is any $F$-stable unipotent class of $\mathcal{G}$ with $\sum_{g \in \mathcal{O}^{\prime} F} \varrho(g) \neq 0$, then

$$
\operatorname{dim} \mathcal{O}^{\prime} \leqslant \operatorname{dim} \mathcal{O}
$$

As $p$ is a good prime for $\mathcal{G}$, each $\varrho \in \operatorname{Irr}\left(\mathcal{G}^{F}\right)$ has a unique unipotent support $\mathcal{O}_{\varrho}[11$, Theorem 1.4].

Next, $\mathcal{O} \cap G$ is a disjoint union $\bigcup_{i=1}^{r} u_{i}^{G}$ of, say, $r$ conjugacy classes in $G$. If

$$
A(x)=\mathbf{C}_{\mathcal{G}}(x) / \mathbf{C}_{\mathcal{G}}(x)^{\circ}
$$

is the component group of the centralizer of $x \in \mathcal{G}$, then one defines

$$
\widetilde{\Gamma}_{u}:=\sum_{i=1}^{r}\left[A\left(u_{i}\right): A\left(u_{i}\right)^{F}\right] \Gamma_{u_{i}} .
$$

Then, $\mathcal{O}$ is called a wave front set for a given $\varrho \in \operatorname{Irr}(G)$ if

(i) $\left[\widetilde{\Gamma}_{u}, \varrho\right]_{G} \neq 0$;

(ii) If $\mathcal{O}^{\prime}=v^{\mathcal{G}}$ is a unipotent class of $\mathcal{G}$ with $v \in \mathcal{G}^{F}$ such that $\left[\widetilde{\Gamma}_{v}, \chi\right]_{G} \neq 0$, then $\operatorname{dim} \mathcal{O}^{\prime} \leqslant \operatorname{dim} \mathcal{O}$.

Work of Lusztig [41] and subsequently [50, Theorem 14.10] show that each $\varrho \in \operatorname{Irr}(G)$ has a unique wave front set $\mathcal{O}_{\varrho}^{*}$. Moreover, if $\mathbf{Z}(\mathcal{G})$ is connected, then $\mathcal{O}_{\varrho}^{*}$ is the unipotent class denoted by $\xi(\varrho)$ in [41, property (13.4.3)], and, if $G$ is defined over $\mathbb{F}_{q}$, then as a polynomial in $q$ with rational coefficients, the degree of $\varrho$ is

$$
\varrho(1)=\frac{1}{n_{\varrho}} q^{\left(\operatorname{dim} \mathcal{O}_{\varrho}^{*}\right) / 2}+\text { lower powers of } q,
$$

for some positive integer $n_{\varrho}$ dividing $|A(u)|$ if $u \in \mathcal{O}_{\varrho}$. Furthermore, if $D_{G}$ denotes the Alvis-Curtis duality (cf. [8, Chapter 8]), and $\varrho^{*}= \pm D_{G}(\varrho) \in \operatorname{Irr}(G)$ for $\varrho \in \operatorname{Irr}(G)$, then

$$
\mathcal{O}_{\varrho^{*}}=\mathcal{O}_{\varrho}^{*}
$$


(see e.g. $[50, \S 1.5])$.

The next two lemmas are well known to the experts. In particular, they have similar conclusions and proofs to Theorems 4.1(ii) and 1.7 of [36]. However, for application to bounding the function $f(r)$ in Theorem 1.1 (see Proposition 2.7), we need the extra detail in the lemmas concerning polynomials being products of cyclotomic polynomials, which is not made explicit in [36]. We omit their proofs.

Lemma 2.3. There is a constant $N=N(r)$ depending only on $r$ and a collection of $N$ monic polynomials, each being a product of cyclotomic polynomials, such that the following statement holds. If $\mathcal{G}$ is a connected reductive group of semisimple rank $\leqslant r$ in characteristic $p, \mathcal{G}^{F}$ is defined over $\mathbb{F}_{q}$, and $s \in \mathcal{G}^{F}$ is semisimple, then

$$
\left[\mathcal{G}^{F}:\left(\mathbf{C}_{\mathcal{G}}(s)^{\circ}\right)^{F}\right]_{p^{\prime}}=f(q),
$$

where $f$ is one of the chosen polynomials.

In what follows, with a slight abuse of language, we also view $t$ as a cyclotomic polynomial in variable $t$.

Lemma 2.4. There are constants $B_{1}=B_{1}(r)$ and $B_{2}=B_{2}(r)$ depending only on $r$, and $B_{2}$ monic polynomials, each being a product of cyclotomic polynomials in one variable $t$, such that the following statement holds for any connected reductive algebraic group $\mathcal{G}$ of semisimple rank $\leqslant r$ with connected center in good characteristic. When $\mathcal{G}^{F}$ is defined over $\mathbb{F}_{q}$ and $\chi \in \operatorname{Irr}\left(\mathcal{G}^{F}\right)$, then

$$
\chi(1)=\frac{1}{n_{\chi}} \operatorname{Deg}_{\chi}^{*}(q),
$$

where $\operatorname{Deg}_{\chi}^{*}$ is one of the chosen monic polynomials, $n_{\chi} \in \mathbb{N}, 1 \leqslant n_{\chi} \leqslant B_{1}$. In fact, if $[\mathcal{G}, \mathcal{G}]$ is simple, then one can take $B_{1}$ to be the largest order of the component group $\mathbf{C}_{\mathcal{H}}(u) / \mathbf{C}_{\mathcal{H}}(u)^{\circ}$, where $\mathcal{H}$ is any simple algebraic group of rank $r$ and $u \in \mathcal{H}$ any unipotent element.

Recall that the set of unipotent classes in $\mathcal{G}$ admits the partial order $\leqslant$, where $u^{\mathcal{G}} \leqslant v^{\mathcal{G}}$ if and only if $u^{\mathcal{G}} \subseteq \overline{v^{\mathcal{G}}}$.

Proposition 2.5. Let $p$ be a good prime for $\mathcal{G}, G=\mathcal{G}^{F}$, and let $u \in G$ be a unipotent element. Then, the following statements hold:

(i) $D_{G}\left(\Gamma_{u}\right)$ is unipotently supported, i.e. is zero on all non-unipotent elements of $G$.

(ii) Suppose that $D_{G}\left(\Gamma_{u}\right)(v) \neq 0$ for some unipotent element $v \in G$. If $\mathbf{Z}(\mathcal{G})$ is disconnected, assume in addition that $q$ is large enough compared to the semisimple rank of $\mathcal{G}$. Then, $u^{\mathcal{G}} \leqslant v^{\mathcal{G}}$. 
Proof. (i) is well known, and (ii) is [7, Scholium 2.3]. (Even though [7] assumes that $p$ is large enough, in fact the proof of [7, Scholium 2.3] needs only that $p$ is a good prime. As pointed out to the authors by J. Michel and J. Taylor, the proof in [7] relies on the validity of the results in [42], which were shown to hold under the indicated hypotheses by Shoji [47]; cf. [48, Theorem 4.2].)

Proposition 2.6. Let $\mathcal{G} / \mathbf{Z}(\mathcal{G})$ be simple, $\mathbf{Z}(\mathcal{G})$ be connected, $p$ be a good prime for $\mathcal{G}$, and let $G=\mathcal{G}^{F}$. Suppose that $\chi \in \operatorname{Irr}(G)$ is such that ${ }^{*} R_{L}^{G}(\chi) \neq 0$ for $L=\mathcal{L}^{F}$, where $\mathcal{L}$ is a proper split Levi subgroup of $\mathcal{G}$, and let $\eta \in \operatorname{Irr}(L)$ be an irreducible constituent of ${ }^{*} R_{L}^{G}(\chi)$. Let $\mathcal{O}_{\chi}^{*}=v^{\mathcal{G}}$ and $\mathcal{O}_{\eta}^{*}=u^{\mathcal{L}}$. Then, $\operatorname{dim} u^{\mathcal{G}} \leqslant \operatorname{dim} v^{\mathcal{G}}$.

Proof. (i) To distinguish between GGGRs for $G$ and $L$, we will add the relevant superscript to their notation, e.g. $\Gamma_{u}^{L}$ is the GGGR of $L$ labeled by $u$. First, we show that, if $R_{L}^{G}\left(D_{L} \Gamma_{u}^{L}\right)(w) \neq 0$ for $w \in G$, then $w$ is unipotent and $u^{\mathcal{G}} \leqslant w^{\mathcal{G}}$. Indeed, by Proposition $2.5(\mathrm{i})$, the generalized character $D_{L} \Gamma_{u}^{L}$ is unipotently supported, whence $R_{L}^{G}\left(D_{L} \Gamma_{u}^{L}\right)$ is also unipotently supported by [8, Proposition 12.2]. In particular, $w$ is unipotent. Recall that $\mathcal{L}$ is a Levi subgroup of an $F$-stable parabolic subgroup $\mathcal{P}$ with unipotent radical $\mathcal{U}$. The condition on $w$ now implies that some $G$-conjugate of $w$ is $w^{\prime}=x y$, where $x \in \mathcal{U}^{F}, y \in L$, and $D_{L} \Gamma_{u}^{L}(y) \neq 0$. By Proposition 2.5 applied to $D_{L} \Gamma_{u}^{L}, y$ is unipotent and $u^{\mathcal{L}} \leqslant y^{\mathcal{L}}$. It then follows by [12, Lemma 5.2] (which is true for any connected reductive group $\mathcal{G}$ ) that

$$
u^{\mathcal{G}} \leqslant y^{\mathcal{G}} \leqslant(x y)^{\mathcal{G}}=w^{\mathcal{G}}
$$

as stated.

(ii) By the assumption, we may assume that $u \in L$ and $\eta$ is an irreducible constituent of the GGGR $\Gamma_{u}^{L}$. It follows that

$$
\begin{aligned}
0 & <\left[{ }^{*} R_{L}^{G}(\chi), \eta\right]_{L} \leqslant\left[{ }^{*} R_{L}^{G}(\chi), \Gamma_{u}^{L}\right]_{L}=\left[\chi, R_{L}^{G}\left(\Gamma_{u}^{L}\right)\right]_{G} \\
& =\left[D_{G}(\chi), D_{G}\left(R_{L}^{G}\left(\Gamma_{u}^{L}\right)\right)\right]_{G}=\left[D_{G}(\chi), R_{L}^{G}\left(D_{L} \Gamma_{u}^{L}\right)\right]_{G} .
\end{aligned}
$$

Here we use the self-adjointness of $D_{G}$ and the intertwining property of $D_{G}$ with $R_{L}^{G}$ (see [8, Proposition 8.10 and Theorem 8.11]). In particular, there must exist some $w \in G$ such that

$$
D_{G}(\chi)(w) \neq 0 \quad \text { and } \quad R_{L}^{G}\left(D_{L} \Gamma_{u}^{L}\right)(w) \neq 0 .
$$

Let $\chi^{*}= \pm D_{G}(\chi) \in \operatorname{Irr}(G)$, so that $\mathcal{O}_{\chi^{*}}=\mathcal{O}_{\chi}^{*}=v^{\mathcal{G}}$, with $v \in G$. By (i), the condition

$$
R_{L}^{G}\left(D_{L} \Gamma_{u}^{L}\right)(w) \neq 0
$$

implies that $w$ is unipotent and

$$
u^{\mathcal{G}} \leqslant w^{\mathcal{G}}
$$


Now we can apply [1, Theorem 8.1] (which uses only the assumption that $\mathbf{Z}(\mathcal{G})$ is connected and $\mathcal{G} / \mathbf{Z}(\mathcal{G})$ is simple; cf. also [50, Corollary 13.6]) to obtain from $\chi^{*}(w) \neq 0$ that

$$
\operatorname{dim} w^{\mathcal{G}} \leqslant \operatorname{dim} v^{\mathcal{G}}
$$

It follows that

$$
\operatorname{dim} u^{\mathcal{G}} \leqslant \operatorname{dim} \overline{w^{\mathcal{G}}}=\operatorname{dim} w^{\mathcal{G}} \leqslant \operatorname{dim} v^{\mathcal{G}}
$$

as desired.

Proof of Theorem 1.1. (i) Denoting $\varrho={ }^{*} R_{L}^{G}(\chi)$, we have by Proposition 2.1 that $|\chi(g)|=|\varrho(g)| \leqslant \varrho(1)$. Hence, it suffices to bound $\varrho(1)$ in terms of $\chi(1)$. Fix the semisimple rank $r$ of $\mathcal{G}$. First, we handle the case where $\mathbf{Z}(\mathcal{G})$ is connected. Note that $\mathcal{H}:=\mathcal{G} / \mathbf{Z}(\mathcal{G})$ is simple (of rank $r$ ) as $[\mathcal{G}, \mathcal{G}]$ is simple. Consider any irreducible constituent $\eta$ of $\varrho$ and let $\mathcal{O}_{\eta}^{*}=u^{\mathcal{L}}$ for some $u \in L$ and $\mathcal{O}_{\chi}^{*}=v^{\mathcal{G}}$ for some $v \in G$. By Proposition 2.6, we have $\operatorname{dim} u^{\mathcal{G}} \leqslant \operatorname{dim} v^{\mathcal{G}}$. On the other hand, $\operatorname{dim} u^{\mathcal{L}} \leqslant \alpha\left(\operatorname{dim} u^{\mathcal{G}}\right)$ by the choice of $\alpha$, and so

$$
\operatorname{dim} u^{\mathcal{L}} \leqslant \alpha\left(\operatorname{dim} v^{\mathcal{G}}\right)
$$

Now, (2.1) and Lemma 2.4 imply that

$$
\eta(1) \leqslant(q+1)^{\left(\operatorname{dim} u^{\mathcal{L}}\right) / 2} \text { and } \quad B_{1} \chi(1) \geqslant(q-1)^{\left(\operatorname{dim} v^{\mathcal{G}}\right) / 2} .
$$

Let $D=D(r)$ denote the largest dimension of unipotent classes in simple algebraic groups of rank $r$. Using (2.3) and noting that $\operatorname{dim} v^{\mathcal{G}}=\operatorname{dim} v^{\mathcal{H}} \leqslant D(r)$, we then get

$$
\eta(1) \leqslant\left(\frac{q+1}{q-1}\right)^{\alpha D / 2} B_{1}^{\alpha} \chi(1)^{\alpha} .
$$

Setting $C:=3^{D / 2}$ and applying Proposition 2.2, we now obtain

$$
\varrho(1) \leqslant A\left(\max _{\eta} \eta(1)\right) \leqslant A B_{1} C \chi(1)^{\alpha},
$$

and we are done in this case.

(ii) Next, we handle the general case, where $\mathbf{Z}(\mathcal{G})$ may be disconnected. Consider a regular embedding of $\mathcal{G}$ into $\widetilde{\mathcal{G}}$ with connected center and with compatible Frobenius $\operatorname{map} F: \widetilde{\mathcal{G}} \rightarrow \widetilde{\mathcal{G}}$, and set $\widetilde{G}:=\widetilde{\mathcal{G}}^{F}$ and $\mathcal{Z}:=\mathbf{Z}(\widetilde{\mathcal{G}})$. As $\widetilde{\mathcal{G}}=\mathcal{Z}[\mathcal{G}, \mathcal{G}], \widetilde{\mathcal{G}}$ and $\mathcal{G}$ have the same semisimple rank. Also, if $\mathcal{L}$ is a Levi subgroup of an $F$-stable parabolic subgroup $\mathcal{P}$ of $\mathcal{G}$, then we can embed $\mathcal{P}$ in the $F$-stable parabolic subgroup $\widetilde{\mathcal{P}}=\mathcal{U} \tilde{\mathcal{L}}=\mathbf{N}_{\widetilde{\mathcal{G}}}(\mathcal{U})$, with the same unipotent radical $\mathcal{U}$ as of $\mathcal{P}$ and with $\tilde{\mathcal{L}}=\mathcal{Z} \mathcal{L}$. Now, set $\tilde{L}:=\tilde{\mathcal{L}}^{F}$ and note that

$$
\widetilde{G}=G \tilde{L}
$$


Consider any $\chi \in \operatorname{Irr}(G)$ and some $\widetilde{\chi} \in \operatorname{Irr}(\widetilde{G})$ lying above $\chi$, and denote

$$
\varrho:={ }^{*} R_{L}^{G}(\chi) \text { and } \quad \tilde{\varrho}:={ }^{*} R_{\tilde{L}}^{\widetilde{G}}(\tilde{\chi}) .
$$

Note that $\widetilde{\mathcal{P}}^{F}=U \tilde{L}$, and by (2.4) we can choose a set of representatives of $G$-cosets in $\widetilde{G}$ that is contained in $\tilde{L}$. Hence, by Clifford's theorem, we can write

$$
\left.\widetilde{\chi}\right|_{G}=\sum_{i=1}^{t} \chi^{x_{i}}
$$

where $1=x_{1}, \ldots, x_{t} \in \tilde{L}$. As $\tilde{L}$ normalizes $U$, we see that the Harish-Chandra restrictions $\varrho_{i}$ of $\chi^{x_{i}}$ to the Levi subgroup $L$ all have the same dimension, equal to $\left[\left.\chi\right|_{U}, 1_{U}\right]_{U}$. Thus,

$$
\varrho(1)=\frac{\tilde{\varrho}(1)}{t} \quad \text { and } \quad \chi(1)=\frac{\tilde{\chi}(1)}{t} .
$$

Now, any unipotent element $v \in \tilde{L}$ is contained in $\tilde{L} \cap \mathcal{G}=L$, and $v^{\widetilde{\mathcal{G}}}=v^{\mathcal{G}}$ and similarly $v^{\tilde{\mathcal{L}}}=v^{\mathcal{Z} \mathcal{L}}=v^{\mathcal{L}}$. Thus the constants $\alpha$ for $L$ and for $\tilde{L}$ as defined in Theorem 1.1 are the same. Applying Lemma 2.1 to $\chi$ and the result of (i) to $\widetilde{\chi}$, we now have

$$
|\chi(g)|=|\varrho(g)| \leqslant \varrho(1)=\frac{1}{t} \tilde{\varrho}(1) \leqslant \frac{1}{t} f(r) \widetilde{\chi}(1)^{\alpha} \leqslant f(r) \chi(1)^{\alpha} .
$$

This completes the proof of Theorem 1.1.

Remark. In the case of $\mathrm{GL}_{n}(q)$, it is possible to give an alternate proof of Theorem 1.1 which does not use recent results on unipotent supports and wave front sets; we do not give this here, but a sketch can be found in the last section of [29].

The next result provides a bound for the function $f$ in Theorem 1.1.

Proposition 2.7. Under the assumptions of Theorem 1.1, suppose that $q \geqslant q_{0} \geqslant 2$. Then, $f(r)$ can be chosen to be

$$
W(r)^{2} B(r)\left(\frac{q_{0}+1}{q_{0}-1}\right)^{(d(r)-r) / 2}
$$

where $W(r)$ is the largest order of the Weyl group of $\mathcal{H}, B(r)$ is the largest order of $A(u)$ for unipotent elements $u \in \mathcal{H}$, and $d(r)$ is the largest dimension of $\mathcal{H}$, when $\mathcal{H}$ runs over simple algebraic groups of rank $r$. In particular, if $r \geqslant 9$ and $q \geqslant r^{2}+1$, one can take

$$
f(r)=2^{2 r+\sqrt{2 r}+3}(r !)^{2} .
$$


Proof. By the proof of Theorem 1.1, we may choose $f(r)=A B_{1} C_{1}$, with

$$
C_{1}=\left(\frac{q_{0}+1}{q_{0}-1}\right)^{(d(r)-r) / 2}
$$

(because $D=d(r)-r)$. Next, $A \leqslant W(r)^{2}$ by Proposition 2.2 and $B_{1} \leqslant B(r)$ by Lemma 2.4. Now, assume that $r \geqslant 9$ and $q_{0} \geqslant r^{2}+1$. Then, $W(r)=2^{r} r$ ! and $d(r)=2 r^{2}+r$, so

$$
\left(\frac{q_{0}+1}{q_{0}-1}\right)^{(d(r)-r) / 2} \leqslant\left(1+\frac{2}{r^{2}}\right)^{r^{2}}
$$

It remains to bound $B(r)$. If $\mathcal{H}=\operatorname{Spin}_{n}(\mathbb{K})$, with $n=2 r$ or $n=2 r+1$, and $u=\sum_{i} J_{i}^{r_{i}}$ is a unipotent element in $\mathcal{H}$ with $r_{i}$ Jordan blocks of size $i \geqslant 1$, then, according to [32, §3.3.5], $|A(u)| \leqslant \max \left(2,2^{k}\right)$, where $k$ is the number of odd $i$ with $r_{i}>0$. Note that

$$
2 r+1 \geqslant \sum_{j=1}^{k}(2 j-1)=k^{2}
$$

and so $|A(u)| \leqslant 2^{\sqrt{2 r+1}}$. Other simple groups of rank $r$ can be analyzed similarly using $\left[32\right.$, Theorem 3.1] and yield smaller bound on $|A(u)|$. Hence, we can take $B(r)=2^{\sqrt{2 r+1}}$ and complete the proof by observing that

$$
\left(1+\frac{2}{r^{2}}\right)^{r^{2}} 2^{\sqrt{2 r+1}}<2^{\sqrt{2 r}+3}
$$

We conclude the section with some examples illustrating the sharpness of the $\alpha$ bound in Theorem 1.1.

Example 2.8. (i) Let $G:=\mathrm{GL}_{n}(q)$ with $q>2$, and let $g=\operatorname{diag}\left(\varepsilon, I_{n-1}\right) \in G$ for some $1 \neq \varepsilon \in \mathbb{F}_{q}^{\times}$. Then, $L:=\mathbf{C}_{G}(g)=\mathrm{GL}_{1}(q) \times \mathrm{GL}_{n-1}(q)$ is a proper split Levi subgroup of $G$.

Now, let $\chi=\varrho_{n}$ denote the unipotent character of $\mathrm{GL}_{n}(q)$ labeled by the partition $(n-1,1)$. Then, $\varrho_{n}(1)=\left(q^{n}-q\right) /(q-1)$. A computation inside the Weyl group of $G$ (using the comparison theorem [18, Theorem 5.9]) shows that

$$
{ }^{*} R_{L}^{G}\left(\varrho_{n}\right)=1_{\mathrm{GL}_{1}(q)} \otimes\left(\varrho_{n-1}+1_{\mathrm{GL}_{n-1}(q)}\right)
$$

Proposition 2.1 implies that

$$
|\chi(g)|=\varrho_{n-1}(1)+1=\frac{q^{n-1}-1}{q-1} \approx \chi(1)^{(n-2) /(n-1)},
$$

if $q$ is large enough. For this Levi subgroup $L$, the value of $\alpha$ in Theorem 1.1 is precisely $(n-2) /(n-1)$ (see Proposition 4.3 ), so the $\alpha$-bound is perfectly sharp in this example. 
(ii) The Steinberg character St of a group $G=\mathcal{G}^{F}$ as in Theorem 1.1 provides a good source of examples, since its values are easily calculated (see [5, Theorem 6.4.7]): for a semisimple element $g \in G$,

$$
|\mathrm{St}(g)|=\left|\mathbf{C}_{G}(g)\right|_{p}
$$

where $p$ is the underlying characteristic.

As a first example, let $G=\mathrm{GL}_{n}(q)$ and let $g=\operatorname{diag}\left(\varepsilon, I_{n-1}\right)$, as in the previous example. Then,

$$
\operatorname{St}(g)=\left|\mathrm{GL}_{n-1}(q)\right|_{p}=q^{(n-1)(n-2) / 2}=\operatorname{St}(1)^{(n-2) / n},
$$

while $\alpha=(n-2) /(n-1)$ for the Levi subgroup $\mathbf{C}_{G}(g)$, as observed above.

As another example, let $G=\mathrm{GL}_{n}(q)$ and suppose that $n=m k$, where $2 \leqslant m \leqslant q-1$ and $k>1$. Let $\lambda_{1}, \ldots, \lambda_{m}$ be distinct elements of $\mathbb{F}_{q}^{\times}$, and define

$$
g=\operatorname{diag}\left(\lambda_{1} I_{k}, \ldots, \lambda_{m} I_{k}\right) \in G
$$

Let $L=\mathbf{C}_{G}(g)=\mathrm{GL}_{k}(q)^{m}$. By Corollary 1.11, $\alpha(L)=1 / m$. On the other hand,

$$
\operatorname{St}(g)=q^{m k(k-1) / 2}=\operatorname{St}(1)^{(k-1) /(m k-1)},
$$

and the exponent $(k-1) /(m k-1)$ is close to $\alpha=1 / m$ for $k$ large and $m$ fixed.

Similar examples showing the sharpness of Theorem 1.1 for the Steinberg character of other classical groups can be constructed using [37, Lemma 3.4].

(iii) Fix $m \geqslant 2$ and consider $G=\mathrm{GL}_{2 m}(q)$ with $q$ large enough (compared to $m$ ). Again let $\lambda_{1}, \ldots, \lambda_{m}$ be distinct elements of $\mathbb{F}_{q}^{\times}$, and define

$$
g=\operatorname{diag}\left(\lambda_{1} I_{2}, \ldots, \lambda_{m} I_{2}\right) \in G
$$

Then, $L=\mathbf{C}_{G}(g)=\mathrm{GL}_{2}(q)^{m}$, and $\alpha=\alpha(L)=1 / m$ as mentioned above. Consider the unipotent characters $\chi^{(2 m-j, j)}$ of $G$ labeled by the partition $(2 m-j, j), 0 \leqslant j \leqslant m$. Then,

$$
\sum_{i=0}^{j} \chi^{(2 m-i, i)}
$$

is the permutation character of $G$ acting on the set of $j$-dimensional subspaces of the natural module $V=\mathbb{F}_{q}^{2 m}$. Note that $g$ fixes $m q^{m-1}\left(1+O\left(q^{-1}\right)\right)(m-1)$-dimensional subspaces of $V$, and $(q+1)^{m}\left(1+O\left(q^{-1}\right)\right) m$-dimensional subspaces of $V$. It follows that, for $\chi:=\chi^{(m, m)}$, we have

$$
\chi(g)=q^{m}\left(1+O\left(q^{-1}\right)\right),
$$

whereas $\chi(1)=q^{m^{2}}(1+O(1 / q))$. Thus, $\chi(g) \approx \chi(1)^{\alpha}$. 
(iv) More generally, fix $k, m \geqslant 2$ and consider $G=\mathrm{GL}_{m k}(q)$ with $q$ large enough (compared to $\max (m, k))$. Again, let $\lambda_{1}, \ldots, \lambda_{m}$ be distinct elements of $\mathbb{F}_{q}^{\times}$, and define

$$
g=\operatorname{diag}\left(\lambda_{1} I_{k}, \ldots, \lambda_{m} I_{k}\right) \in G
$$

Then, $L=\mathbf{C}_{G}(g)=\mathrm{GL}_{k}(q)^{m}$, and $\alpha=\alpha(L)=1 / m$ as mentioned above. Consider the unipotent character $\chi:=\chi^{\mu}$ of $G$ labeled by the partition $\mu:=\left(m^{k}\right) \vdash m k$. Observe that ${ }^{*} R_{L}^{G}(\chi)$ contains the Steinberg character $\mathrm{St}_{L}$ of $L$. (Indeed, by [16, Proposition 5.3], the Alvis-Curtis duality functor $D_{G}$ sends $\chi$ to $\pm \chi^{\nu}$, where $\nu=\mu^{\prime}=\left(k^{m}\right) \vdash m k$, whereas $D_{L}\left(1_{L}\right)=\mathrm{St}_{L}$. Now, by [8, Corollary 8.13], we have

$$
\begin{aligned}
{\left[{ }^{*} R_{L}^{G}(\chi), \mathrm{St}_{L}\right]_{L} } & =\left[{ }^{*} R_{L}^{G}\left( \pm D_{G}\left(\chi^{\nu}\right)\right), \mathrm{St}_{L}\right]_{L}= \pm\left[D_{L}\left({ }^{*} R_{L}^{G}\left(\chi^{\nu}\right)\right), D_{L}\left(1_{L}\right)\right]_{L} \\
& = \pm\left[{ }^{*} R_{L}^{G}\left(\chi^{\nu}\right), 1_{L}\right]_{L}= \pm\left[\chi^{\nu}, R_{L}^{G}\left(1_{L}\right)\right]_{G}
\end{aligned}
$$

But note that $L$ is a Levi subgroup of a rational parabolic subgroup of type $\nu$ of $\mathrm{GL}_{m k}(\mathbb{K})$, whence $\chi^{\nu}$ is an irreducible constituent of $R_{L}^{G}\left(1_{L}\right)$, and the claim follows.) Since $\chi$ is a unipotent character and the Harish-Chandra restriction preserves rational series, every irreducible constituent of ${ }^{*} R_{L}^{G}(\chi)$ is a unipotent character of $L$, and so contains $g \in \mathbf{Z}(L)$ in its kernel. It now follows from Proposition 2.1 that

$$
\chi(g)={ }^{*} R_{L}^{G}(\chi)(g)={ }^{*} R_{L}^{G}(\chi)(1) \geqslant \mathrm{St}_{L}(1)=q^{m k(k-1) / 2} .
$$

On the other hand, the degree formula $[5, \S 13.8]$ implies that

$$
\chi(1)=q^{m^{2} k(k-1) / 2}\left(1+O\left(q^{-1}\right)\right),
$$

and we again obtain that $\chi(g) \gtrsim \chi(1)^{\alpha}$.

(v) As far as the exceptional groups of Lie type are concerned, it is again interesting to use the Steinberg character to test the sharpness of Theorem 1.1. For example, let $G=E_{8}(q)$, and suppose $g \in G$ is a semisimple element with a Levi subgroup of type $E_{7}$ as centralizer. Then,

$$
\operatorname{St}(g)=\left|E_{7}(q)\right|_{p}=q^{63}=\operatorname{St}(1)^{\beta},
$$

where $\beta=\frac{21}{40}$, while the $\alpha$-value of this Levi is $\frac{17}{29}$, by Theorem 1.7 . One can calculate such $\beta$-values for all the Levi subgroups in Table 1 of Theorem 1.7; it is never the case that $\beta=\alpha$, but in some cases the values of $\beta$ and $\alpha$ are quite close.

(vi) We offer one more example with $G=\mathcal{G}^{F}=\operatorname{SL}_{n}(q)$, with $q \geqslant n+2$, and

$$
g=\operatorname{diag}\left(\lambda_{1}, \lambda_{2}, \ldots, \lambda_{n}\right) \in G
$$


where $\lambda_{1}, \ldots, \lambda_{n} \in \mathbb{F}_{q}^{\times}$are pairwise distinct. Then, $T=\mathbf{C}_{G}(g)$ is a maximally split maximal torus. Let $\mu \vdash n$ be such that the irreducible character $S^{\mu}$ of the Weyl group $W(\mathcal{G}) \cong \mathrm{S}_{n}$ labeled by $\mu$ has the largest possible degree, and let $\chi:=\chi^{\mu}$ denote the unipotent character of $G$ labeled by $\mu$. As in (iv), every irreducible constituent of ${ }^{*} R_{T}^{G}(\chi)$ contains $g$ in its kernel. A computation in $W(\mathcal{G})$ and Proposition 2.1 show that

$$
\chi(g)={ }^{*} R_{T}^{G}(\chi)(g)={ }^{*} R_{T}^{G}(\chi)(1)=S^{\mu}(1),
$$

whereas $\alpha(T)=0$. Thus, for the function $f$ in Theorem 1.1 we have

$$
f(n-1) \geqslant S^{\mu}(1) \geqslant e^{-1.283 \sqrt{n}} \sqrt{n !},
$$

with the latter following from the main result of [38] and [53].

\section{General and special linear groups}

In this section we prove Theorems 1.3, 1.4 and 1.5. Along the way, we establish character bounds for unipotent elements of $\mathrm{GL}_{n}(q)$ (see Theorem 3.3), and also for elements $g=$ $s u=u s$ with extension-field centralizers for their semisimple parts $s$ (Theorem 3.2).

\subsection{Proof of Theorem 1.4}

We will keep the notation of $\S 2$.

(i) First, we consider the case $\mathcal{G}=\mathrm{GL}_{n}(\mathbb{K})$. In this case, the centralizer of any element in $\mathcal{G}$ is connected, and one can check (e.g. using [15]) that $n_{\varrho}=1$ in (2.1). Let $\varphi$ be an irreducible $\ell$-Brauer character of $G=\mathcal{G}^{F}=\mathrm{GL}_{n}(q)$ and $g \in G$ be as in Theorem 1.4. By Proposition 2.1, $|\varphi(g)|=|\psi(g)|$ for $\psi:={ }^{*} R_{L}^{G}(\varphi)$. According to [4, Theorem B], one can label complex and $\ell$-Brauer characters of $G$ and find a complex character $\chi \in \operatorname{Irr}(G)$ with the same label as of $\varphi$ such that both the generic degree of $\chi$ and the lower bound (given in [4, Theorem B]) are monic polynomials in $q$ of same degree say $N_{\chi}$. Using (2.1) and the equality $n_{\chi}=1$, we have

$$
N_{\chi}:=\frac{1}{2} \operatorname{dim} \mathcal{O}_{\chi}^{*}
$$

As $\chi(1)$ is a product of cyclotomic polynomials in $q$, we also have that

$$
\chi(1) \leqslant(q+1)^{N_{\chi}} .
$$

Furthermore, one can easily check that the lower bound in [4, Theorem B] satisfies

$$
\varphi(1) \geqslant(q-1)^{N_{\chi}} .
$$


As $N_{\chi} \leqslant \operatorname{dim} \mathcal{G}=n^{2}$, there is a constant $D=D(n) \leqslant 3^{n^{2}}$ such that

$$
\frac{\chi(1)}{\varphi(1)} \leqslant D .
$$

Since $\chi$ and $\varphi$ have the same labeling, $\varphi$ is a constituent of the restriction $\chi^{\circ}$ of $\chi$ to $\ell^{\prime}$-elements of $G$. Let $\mathcal{P}=\mathcal{U} \mathcal{L}$ be an $F$-stable parabolic subgroup of $\mathcal{G}$ with Levi subgroup $\mathcal{L}$ and unipotent radical $\mathcal{U}$. As $U:=\mathcal{U}^{F}$ is an $\ell^{\prime}$-group, we also have that

$$
\psi(1)=\left[\left.\varphi\right|_{U}, 1_{U}\right]_{U} \leqslant\left[\left.\chi\right|_{U}, 1_{U}\right]_{U}=\varrho(1),
$$

where $\varrho:={ }^{*} R_{L}^{G}(\chi)$. The proof of Theorem 1.1 yields a function $f: \mathbb{N} \rightarrow \mathbb{N}$ such that

$$
\varrho(1) \leqslant f(n) \chi(1)^{\alpha}
$$

Choosing $h$ such that $h(n) \geqslant f(n) D$ and applying (3.1), we obtain

$$
|\varphi(g)|=|\psi(g)| \leqslant \psi(1) \leqslant \varrho(1) \leqslant h(n) \varphi(1)^{\alpha},
$$

as desired.

(ii) Embed $\mathrm{SL}_{n}(q)$ in $\mathrm{GL}_{n}(q)$ and argue as in part (ii) of the proof of Theorem 1.1.

\subsection{Proof of Theorem 1.3}

(i) We first consider the case $G=\mathrm{GL}_{n}(q)$. If $\mathcal{L}$ is a torus, we can choose a regular semisimple element $g \in L$ and take $\chi=1_{G}$. Assume now that $\mathcal{L}$ is not a torus, and choose $u \in L_{\text {unip }}$ such that

$$
\alpha(L)=\frac{\operatorname{dim} u^{\mathcal{L}}}{\operatorname{dim} u^{\mathcal{G}}} .
$$

We may assume that

$$
L=\mathrm{GL}_{n_{1}}(q) \times \mathrm{GL}_{n_{2}}(q) \times \ldots \times \mathrm{GL}_{n_{r}}(q) \text { and } u=\operatorname{diag}\left(u_{1}, u_{2}, \ldots, u_{r}\right),
$$

where $n_{1} \geqslant n_{2} \geqslant \ldots \geqslant n_{r} \geqslant 1$; furthermore, $u_{i} \in \mathrm{GL}_{n_{i}}(q)$ is a unipotent element, the sizes of whose Jordan blocks form a partition $\lambda_{i} \vdash n_{i}$. Let $\mu_{i} \vdash n_{i}$ be the partition conjugate to $\lambda_{i}$ and let $\chi^{\mu_{i}}$ be the unipotent character of $\mathrm{GL}_{n_{i}}(q)$ labeled by $\mu_{i}$. Now Green's formula for the degree of $\chi^{\mu_{i}}$ (see the discussion before [4, Theorem A]) implies that $\chi^{\mu_{i}}(1)$ is a monic polynomial in $q$ of degree $\frac{1}{2} \operatorname{dim} u_{i}^{\mathcal{L}}$. Hence, if we choose $C_{n}$ large enough, then using Lemma 2.3 we see that

$$
\gamma(1) \geqslant \frac{1}{2} q^{\left(\operatorname{dim} u^{\mathcal{L}}\right) / 2}
$$


for $\gamma:=\chi^{\mu_{1}} \otimes \chi^{\mu_{2}} \otimes \ldots \otimes \chi^{\mu_{r}} \in \operatorname{Irr}(L)$ whenever $q \geqslant C_{n}$.

Next, let $\mu:=\mu_{1}+\mu_{2}+\ldots+\mu_{r}$, where we have added zero parts to $\mu_{i}$ so that $\mu_{1}, \ldots, \mu_{r}$ have the same number of parts, and then take the $i$ th part of $\mu$ to be the sum of all the $i$ th parts of $\mu_{1}, \ldots, \mu_{r}$. Again, using Green's formula, we then see that the unipotent character $\chi=\chi^{\mu}$ of $G$ labeled by $\mu$ is a monic polynomial in $q$ of degree $\frac{1}{2} \operatorname{dim}^{u^{\mathcal{G}}}$, whence

$$
\chi(1) \leqslant 2 q^{\left(\operatorname{dim} u^{\mathcal{G}}\right) / 2}
$$

if $q \geqslant C_{n}$.

For any $\nu \vdash m$, let $S^{\nu}$ denote the irreducible character of $\mathrm{S}_{m}$ labeled by $\nu$. An application of the Littlewood-Richardson formula [21, Corollary 2.8.14] shows that the restriction of $S^{\mu}$ to $\mathrm{S}_{n_{1}} \times \mathrm{S}_{n_{2}} \times \ldots \times \mathrm{S}_{n_{r}}$ contains $S^{\mu_{1}} \otimes S^{\mu_{2}} \otimes \ldots \otimes S^{\mu_{r}}$. This computation in the Weyl groups of $\mathcal{G}$ and $\mathcal{L}$ implies that $\gamma$ is an irreducible constituent of ${ }^{*} R_{L}^{G}(\chi)$. Now, if $q \geqslant n+1$, then we can choose a semisimple element $g \in \mathbf{Z}(L)$ such that $\mathbf{C}_{G}(g)=L$. (Indeed, with $L$ given in (3.3), we can fix $m \leqslant n<q$ distinct elements $\lambda_{1}, \ldots, \lambda_{m} \in \mathbb{F}_{q}^{\times}$and choose $g \in G$ that has $\lambda_{i}$ as an eigenvalue with multiplicity $n_{i}$.)

As in Example 2.8 (iv), we have that every irreducible constituent of ${ }^{*} R_{L}^{G}(\chi)$ has $g$ in its kernel, and so, by Proposition 2.1,

$$
\chi(g)={ }^{*} R_{L}^{G}(\chi)(g)={ }^{*} R_{L}^{G}(\chi)(1) \geqslant \gamma(1) .
$$

Hence, the statement follows from the choice (3.2) of $u$ and the bounds (3.4) and (3.5).

(ii) To handle the case of $\mathrm{SL}_{n}(q)$, we first recall that any unipotent character of $G$ remains irreducible over $\mathrm{SL}_{n}(q)$. Furthermore, as mentioned in the proof of Theorem 1.1, for any proper split Levi subgroup $\mathcal{L}$ of $\mathcal{G}=\mathrm{GL}_{n}(\mathbb{K})$ we have

$$
\mathcal{G}=[\mathcal{G}, \mathcal{G}] \mathcal{Z} \quad \text { and } \quad \mathcal{L}=(\mathcal{L} \cap[\mathcal{G}, \mathcal{G}]) \mathcal{Z}
$$

where $\mathcal{Z}:=\mathbf{Z}(\mathcal{G})$. It follows that

$$
\operatorname{dim} u^{\mathcal{G}}=\operatorname{dim} u^{[\mathcal{G}, \mathcal{G}]} \quad \text { and } \quad \operatorname{dim} u^{\mathcal{L}}=\operatorname{dim} u^{\mathcal{L} \cap[\mathcal{G}, \mathcal{G}]}
$$

for any element $u \in \mathcal{L}_{\text {unip }}=(\mathcal{L} \cap[\mathcal{G}, \mathcal{G}])_{\text {unip }}$. Finally, the condition that $(n !)^{n}$ divides $q-1$ implies, by Lemma 3.1 below, that, for any non-toral Levi subgroup $L$ given in (3.3), we can find an element $g \in \mathrm{SL}_{n}(q)$ with $\mathbf{C}_{G}(g)=L$. Now, the statement for $\mathrm{SL}_{n}(q)$ follows from (i).

LEMma 3.1. Let $1 \leqslant n_{1} \leqslant n_{2} \leqslant \ldots \leqslant n_{r}$, with $r, n_{r} \geqslant 2, n=\sum_{i=1}^{r} n_{i}$, and let $q$ be a prime power such that $N:=n_{r} \prod_{i=1}^{r-1}\left(n_{i}+1\right)$ divides $q-1$. Then, for

$$
L:=\mathrm{GL}_{n_{1}}(q) \times \mathrm{GL}_{n_{2}}(q) \times \ldots \times \mathrm{GL}_{n_{r}}(q)<G:=\mathrm{GL}_{n}(q),
$$

there exists a semisimple element $s \in \mathrm{SL}_{n}(q)$ such that $\mathbf{C}_{G}(s)=L$. 
Proof. Choose $\zeta \in \mathbb{F}_{q}^{\times}$of order $N$, and for any $d \mid N$ let $\zeta_{d}:=\zeta^{N / d}$. Define

$$
h_{i}=\zeta_{\left(n_{1}+1\right)\left(n_{2}+1\right) \ldots\left(n_{i}+1\right)}^{-1} I_{n_{i}} \in \mathrm{GL}_{n_{i}}(q), 1 \leqslant i \leqslant r-1,
$$

and

$$
h_{r}=\zeta I_{n_{r}} \in \mathrm{GL}_{n_{r}}(q) .
$$

We prove by induction on $1 \leqslant i \leqslant r-1$ that $\prod_{j=1}^{i} \operatorname{det}\left(h_{i}\right)=\zeta_{\left(n_{1}+1\right)\left(n_{2}+1\right) \ldots\left(n_{i}+1\right)}$. The induction base $i=1$ is obvious. For the induction step from $i-1$ to $i \geqslant 2$, we have

$$
\prod_{j=1}^{i} \operatorname{det}\left(h_{i}\right)=\zeta_{\left(n_{1}+1\right)\left(n_{2}+1\right) \ldots\left(n_{i-1}+1\right)} \zeta_{\left(n_{1}+1\right)\left(n_{2}+1\right) \ldots\left(n_{i}+1\right)}^{-n_{i}}=\zeta_{\left(n_{1}+1\right)\left(n_{2}+1\right) \ldots\left(n_{i}+1\right)} .
$$

Hence, for $s:=\operatorname{diag}\left(h_{1}, h_{2}, \ldots, h_{r}\right) \in \mathbf{Z}(L)$, we have

$$
\operatorname{det}(s)=\zeta_{\left(n_{1}+1\right)\left(n_{2}+1\right) \ldots\left(n_{r-1}+1\right)} \zeta^{-n_{r}}=1 .
$$

The construction of $s$ and the condition $n_{r} \geqslant 2$ ensure that $\mathbf{C}_{G}(s)=L$.

\subsection{Elements with extension-field centralizers}

TheOREM 3.2. Let $G=\mathrm{GL}_{n}(q)$, with $n \geqslant 2$ and $q \geqslant 8$, and let $g=s u=u s$, with $s \in$ $G$ semisimple and $u \in G$ unipotent. Suppose that $\mathbf{C}_{G}(s) \cong \mathrm{GL}_{n / k}\left(q^{k}\right)$ for some $1<k \mid n$. Then,

$$
\frac{|\chi(g)|}{\chi(1)} \leqslant \frac{f(n)}{q^{n / 3}}
$$

for any $\chi \in \operatorname{Irr}(G)$, with $\chi(1)>1$ and $f(n)=\left(\frac{11}{7}\right)^{n}-\frac{13}{10}$. In particular, if $q \geqslant 227$, then $|\chi(g)| \leqslant \chi(1)^{1-1 / 2 n}$ for all $\chi \in \operatorname{Irr}(G)$.

Proof. We proceed by induction on $n \geqslant 2$. Let $L:=\mathrm{SL}_{n}(q)$ and let $W=\mathbb{F}_{q}^{n}$ denote the natural $G$-module. Since $\chi(1)>1$, all irreducible constituents of $\chi_{L}$ are non-trivial. In particular, if $n \geqslant 3$ then $\chi(1) \geqslant\left(q^{n}-q\right) /(q-1)>q^{n-1}$ by [52, Theorem 1.1].

(i) First, we consider the case $k=n$. Since $\left|\mathbf{C}_{G}(g)\right| \leqslant\left|\mathbf{C}_{G}(s)\right|=q^{n}-1$, we have that $|\chi(g)| \leqslant \sqrt{\left|\mathbf{C}_{G}(g)\right|}<q^{n / 2} ;$ in particular,

$$
\frac{|\chi(g)|}{\chi(1)} \leqslant q^{1-n / 2} \leqslant q^{-n / 3}
$$

if $n \geqslant 6$, or if $n=5$ and $\chi(1) \geqslant q^{5}$. The condition $\mathbf{C}_{G}(s) \cong \mathrm{GL}_{1}\left(q^{n}\right)$ also implies that no eigenvalue of $g$ on $W$ can belong to $\mathbb{F}_{q}$. 
Assume now that $n=5$ and $\chi(1)<q^{5}$. By [52, Theorem 3.1], every irreducible constituent of $\chi_{L}$ is one of the $q-1$ Weil characters, of degree $\left(q^{n}-1\right) /(q-1)-\delta$ with $\delta=0$ or 1. Since Weil characters of $L$ extend to Weil characters of $G, \chi$ is a Weil character. Since no eigenvalue of $g$ on $W$ belongs to $\mathbb{F}_{q}$, using the well-known character formula for Weil characters of $G$, see e.g. [51, formula (1.1)], we now see that $|\chi(g)| \leqslant q+1$, and so

$$
\frac{|\chi(g)|}{\chi(1)}<\frac{q^{2}-1}{q^{5}-q}<q^{-n / 3} .
$$

Consider the case $n=4$. If $\chi(1) \geqslant \frac{1}{2}(q-1)\left(q^{3}-1\right)$, then

$$
\frac{|\chi(g)|}{\chi(1)} \leqslant \frac{\left(q^{4}-1\right)^{1 / 2}}{\left(q^{3}-1\right)(q-1) / 2}<q^{-n / 3}
$$

as $q \geqslant 8$. Assume now that $\chi(1)<\frac{1}{2}\left(q^{3}-1\right)(q-1)$. By [52, Theorem 3.1], every irreducible constituent of $\chi_{L}$ is one of the $q-1$ Weil characters, all of which extend to Weil characters of $G$. Arguing as in the previous case, we see that

$$
\frac{|\chi(g)|}{\chi(1)}<\frac{q^{2}-1}{q^{4}-q}<q^{-n / 3} .
$$

If $n=3$, then, inspecting the character table of $G$ [49], we get

$$
\frac{|\chi(g)|}{\chi(1)} \leqslant \max \left(\frac{1}{q(q+1)}, \frac{3}{\left(q^{2}-1\right)(q-1)}\right)<q^{-n / 3} .
$$

Similarly, for $n=2$, we have $|\chi(g)| / \chi(1) \leqslant 2 /(q-1)<(1.15) q^{-n / 3}$ as $q \geqslant 8$.

Note that $f(n)>1.16$ for all $n \geqslant 2$. Hence, to complete the induction base $2 \leqslant n \leqslant 5$, it remains to consider the case $(n, k)=(4,2)$. Again inspecting the character table of $G$ [49], we see that

$$
\frac{|\chi(g)|}{\chi(1)}<\frac{1}{(q-1)^{2}}<q^{-n / 3} .
$$

(ii) From now on, we may assume that $n \geqslant 6$ and $2 \leqslant k<n$. Consider the action of $u$ on the natural module $W^{\prime}=\mathbb{F}_{q^{k}}^{n / k}$ of $\mathbf{C}_{G}(s)$. If this action induces an element with only one Jordan block, then $\left|\mathbf{C}_{G}(g)\right|=\left|\mathbf{C}_{\mathrm{GL}_{n / k}\left(q^{k}\right)}(u)\right|<q^{n}$, and again (3.6) holds. Thus, we may assume that the $\langle g\rangle$-module $W^{\prime}$ is decomposable as a direct sum of two $\langle g\rangle$-submodules: $W^{\prime}=W_{1}^{\prime} \oplus W_{2}^{\prime}$, with

$$
\operatorname{dim}_{\mathbb{F}_{q^{k}}} W_{1}^{\prime}=a \geqslant \frac{n}{2 k} \quad \text { and } \quad \operatorname{dim}_{\mathbb{F}_{q^{k}}} W_{2}^{\prime}=b \geqslant 1 .
$$

Viewing $W_{i}^{\prime}$ as vector spaces over $\mathbb{F}_{q}$, we get a $g$-invariant decomposition

$$
W=W_{1} \oplus W_{2},
$$


with

$$
\operatorname{dim} W_{1}=a k \geqslant \frac{1}{2} n \quad \text { and } \quad \operatorname{dim} W_{2}=b k \geqslant 2 .
$$

Writing $g=\operatorname{diag}\left(g_{1}, g_{2}\right)$, with $g_{i} \in G_{i}:=\mathrm{GL}\left(W_{i}\right)$, and letting $s_{i}$ denote the semisimple part of $g_{i}$, we have

$$
\mathbf{C}_{G_{1}}\left(s_{1}\right)=\mathrm{GL}\left(W_{1}^{\prime}\right) \cong \mathrm{GL}_{a}\left(q^{k}\right) \quad \text { and } \quad \mathbf{C}_{G_{2}}\left(s_{2}\right)=\mathrm{GL}\left(W_{2}^{\prime}\right) \cong \mathrm{GL}_{b}\left(q^{k}\right)
$$

In particular, the induction hypothesis applies to the elements $g_{i} \in G_{i}$.

(iii) Let $V$ be a $\mathbb{C} G$-module affording the character $\chi$, and denote $L_{i}:=\left[G_{i}, G_{i}\right]$ for $i=1,2$. We decompose the $\left(G_{1} \times G_{2}\right)$-module $V$ as

$$
V=V_{1} \oplus V_{2} \oplus V_{3}
$$

where $V_{1}:=\mathbf{C}_{V}\left(L_{1}\right)$, every irreducible constituent of $V_{2}$ is trivial on $L_{2}$ but not on $L_{1}$, and every irreducible constituent of $V_{3}$ is non-trivial on $L_{1}$ and on $L_{2}$. Let $\chi_{j}$ denote the $G_{1} \times G_{2}$-character afforded by $V_{j}$, for $1 \leqslant j \leqslant 3$.

If $\alpha \otimes \beta$ is any irreducible constituent of $\chi_{3}$, then $\alpha(1), \beta(1)>1$, by the construction of $V_{3}$, whence

$$
\frac{\left|\alpha\left(g_{1}\right)\right|}{\alpha(1)} \leqslant f(a k) q^{-a k / 3} \text { and } \frac{\left|\beta\left(g_{2}\right)\right|}{\beta(1)} \leqslant f(b k) q^{-b k / 3},
$$

by the induction hypothesis applied to $g_{1} \in G_{1}$ and $g_{2} \in G_{2}$. It follows that

$$
\frac{\left|\chi_{3}(g)\right|}{\chi_{3}(1)} \leqslant f(a k) f(b k) q^{-n / 3} .
$$

Next, let $\alpha \otimes \beta$ be any irreducible constituent of $\chi_{2}$. Then, $\alpha(1)>1$ and $\beta(1)=1$, by the construction of $V_{2}$, whence

$$
\frac{\left|\alpha\left(g_{1}\right)\right|}{\alpha(1)} \leqslant f(a k) q^{-a k / 3}, \quad\left|\beta\left(g_{2}\right)\right|=1
$$

by the induction hypothesis applied to $g_{1} \in G_{1}$. It follows that

$$
\left|\chi_{2}(g)\right| / \chi_{2}(1) \leqslant f(a k) q^{-a k / 3} .
$$

(iv) We will now estimate $\chi_{j}(1) / \chi(1)$ for $j=1,2$. Let $d(X)$ denote the smallest degree of a non-trivial complex representation of a finite group $X$, and let

$$
a_{m, q}:=\frac{\sqrt{q-1}}{d\left(\mathrm{SL}_{m}(q)\right)}+\frac{1}{d\left(\mathrm{SL}_{m-1}(q)\right)} \quad \text { and } \quad b_{m, q}:=\sum_{i=m+1}^{\infty} a_{i, q} .
$$


The proof of [26, Proposition 4.2.3] shows that, if $U$ is any non-trivial irreducible $\mathbb{C S L}_{n}(q)$ module for $n>m \geqslant 3$ and $\mathrm{SL}_{m}(q)$ is embedded naturally in $\operatorname{SL}_{n}(q)$, then

$$
\operatorname{dim} \mathbf{C}_{U}\left(\mathrm{SL}_{m}(q)\right) \leqslant b_{m, q} \operatorname{dim} U
$$

By [52, Theorem 1.1], for $m \geqslant 4$ we have $a_{m, q}<(q+\sqrt{q-1}) / q^{m-2}(q+1)$. It follows that

$$
b_{m, q}<\frac{q+\sqrt{q-1}}{q+1} \sum_{i=m-1}^{\infty} q^{-i}=\frac{q(q+\sqrt{q-1})}{q^{m-1}\left(q^{2}-1\right)}<\frac{1.36}{q^{m-1}}
$$

if $m \geqslant 3$ and $q \geqslant 8$. As $a_{3, q}=\sqrt{q-1} /\left(q^{2}+q\right)+e /(q-1)$ with $e:=3-\operatorname{gcd}(q, 2)$ and $q \geqslant 8$, we then have

$$
b_{2, q}=a_{3, q}+b_{3, q}<1.3 q^{-2 / 3} .
$$

Now, since $\frac{1}{2} a k \geqslant n \geqslant 3$, we have $a k-1 \geqslant \frac{1}{3} n$. Applying (3.9) and (3.10), we get

$$
\frac{\chi_{1}(1)}{\chi(1)} \leqslant b_{a k}(q)<1.36 q^{-n / 3}
$$

Similarly,

$$
\frac{\chi_{2}(1)}{\chi(1)} \leqslant b_{b k}(q)<1.36 q^{-(b k-1)} \leqslant 0.17 q^{-b k / 3}
$$

if $b k \geqslant 3$, and

$$
\chi_{2}(1) / \chi(1) \leqslant b_{2}(q)<1.3 q^{-2 / 3},
$$

if $b k \geqslant 2$ (using (3.11) instead of (3.10)). Note that, in the case $b k=2$, we must have $6 \leqslant n=a k+2$, and so $a k \geqslant 4$ and $a k-1 \geqslant 1+\frac{1}{3} n$, whence, instead of (3.12), we have

$$
\frac{\chi_{1}(1)}{\chi(1)} \leqslant b_{a k}(q)<0.17 q^{-n / 3}
$$

(v) Now, if $b k \geqslant 3$, then putting (3.7), (3.8), (3.12), (3.13) together, we obtain

$$
|\chi(g)| \leqslant\left|\chi_{1}(1)\right|+\left|\chi_{2}(g)\right|+\left|\chi_{3}(g)\right| \leqslant \frac{\chi(1)}{q^{n / 3}}(1.36+0.17 f(a k)+f(a k) f(b k)) .
$$

If $b k=2$, then (3.7), (3.8), (3.14), (3.15) altogether imply

$$
|\chi(g)| \leqslant\left|\chi_{1}(1)\right|+\left|\chi_{2}(g)\right|+\left|\chi_{3}(g)\right| \leqslant \frac{\chi(1)}{q^{n / 3}}(0.17+1.3 f(a k)+f(a k) f(b k)) .
$$

The choice $f(n)=\left(\frac{11}{7}\right)^{n}-1.3$ ensures that

$$
f(n)=f(a k) f(b k)+1.3 f(a k)+1.3 f(b k)+0.39>f(a k) f(b k)+0.17 f(a k)+1.36,
$$

whence $|\chi(g)| / \chi(1) \leqslant f(n) q^{-n / 3}$, completing the induction step of the proof. The last statement then follows, since $f(n)<q^{n / 12}$ when $q \geqslant 227$ and $\chi(1)<q^{n^{2} / 2}$. 


\subsection{Unipotent elements in general linear groups}

THEOREM 3.3. There is a function $g: \mathbb{N} \rightarrow \mathbb{N}$ such that the following statement holds. For any $n \geqslant 2$, any prime power $q, \ell=0$ or $\ell$ being any prime not dividing $q$, any irreducible $\ell$-Brauer character $\varphi$ of $G:=\mathrm{GL}_{n}(q)$, and any unipotent element $1 \neq u \in G$,

$$
|\varphi(u)| \leqslant g(n) \varphi(1)^{(n-2) /(n-1)} .
$$

Proof. Note that the statement holds when $n=2$ (choosing $g(2)=1)$ as in this case we have $|\varphi(u)| \leqslant 1$. So, in what follows, we may assume that $n \geqslant 3$.

Recall the partial order $\leqslant$ on the set of unipotent classes of $\mathcal{G}=\mathrm{GL}_{n}(\mathbb{K}): x^{\mathcal{G}} \leqslant y^{\mathcal{G}}$ precisely when $x^{\mathcal{G}} \subseteq \overline{y^{\mathcal{G}}}$, and we consider $G=\mathcal{G}^{F}$ for a suitable Frobenius endomorphism $F$. Note that the unipotent classes in $\mathcal{G}$ are parameterized by partitions of $n$. We will prove by induction using the partial order $\leqslant$ that, if $u$ is parameterized by a partition $\lambda \vdash n$, then

$$
|\varphi(g)| \leqslant g_{\lambda}(n) \varphi(1)^{(n-2) /(n-1)}
$$

for some positive constant $g_{\lambda}(n)$ depending only on $\lambda$. Then, the statement follows by taking

$$
g(n):=\max _{\lambda \vdash n} g_{\lambda}(n) .
$$

Observe that $u$ is a Richardson unipotent element, that is, we can find an $F$-stable parabolic subgroup $\mathcal{P}$ with unipotent radical $\mathcal{U}$ such that $u^{\mathcal{G}} \cap \mathcal{U}$ is an open dense subset of $\mathcal{U}$ that forms a single $\mathcal{P}$-orbit. Indeed, as shown in [19, §5.5], if $\mu=\left(\mu_{1}, \ldots, \mu_{l}\right) \vdash n$ is the conjugate partition associated with $\lambda$, then one can just take $\mathcal{P}$ to be the standard parabolic subgroup generated by the upper-triangular Borel subgroup together with matrices in the block-diagonal form, with block sizes $\mu_{1} \times \mu_{1}, \ldots, \mu_{l} \times \mu_{l}$. Furthermore, $\mathbf{C}_{\mathcal{G}}(u)$ is connected (as $\mathcal{G}=\mathrm{GL}_{n}(\mathbb{K})$ ), of dimension equal to $\operatorname{dim} \mathcal{P}-\operatorname{dim} \mathcal{U}$, and contained in $\mathcal{P}$; see [5, Corollary 5.2.2]. Since $u^{\mathcal{G}} \cap \mathcal{U}$ is a single $\mathcal{P}$-orbit and $\mathbf{C}_{\mathcal{G}}(u)=\mathbf{C}_{\mathcal{G}}(u)^{\circ}=\mathbf{C}_{\mathcal{P}}(u)$ is connected, by the Lang-Steinberg theorem, $u^{\mathcal{G}} \cap \mathcal{U}$ contains an $F$-stable element $u^{\prime}$, i.e. $u^{\prime} \in u^{\mathcal{G}} \cap U$ for $U:=\mathcal{U}^{F}$. The connectedness of $\mathbf{C}_{\mathcal{G}}(u)$ implies, by the Lang-Steinberg theorem, that $u, u^{\prime} \in u^{\mathcal{G}} \cap U$ are $G$-conjugate. Replacing $u$ by $u^{\prime}$, we may assume that $u \in u^{\mathcal{G}} \cap U$. Then, applying again the Lang-Steinberg theorem, we see that any element $w \in u^{\mathcal{G}} \cap U$ can be written as $h u h^{-1}$ for some $h \in P:=\mathcal{P}^{F}$. Conversely, the $P$-orbit of $u$ is contained in $u^{\mathcal{G}} \cap U$. Thus $u^{\mathcal{G}} \cap U$ is a single $P$-orbit, and so

$$
\left|u^{\mathcal{G}} \cap U\right|=[P: C],
$$

where $C:=\mathbf{C}_{P}(u)=\mathbf{C}_{\mathcal{P}}(u)^{F}=\mathbf{C}_{\mathcal{G}}(u)^{F}$. The structure of $\mathbf{C}_{\mathcal{G}}(u)$ is given in [32, Theorem 3.1]. As $\operatorname{dim} \mathbf{C}_{\mathcal{G}}(u)=\operatorname{dim} \mathcal{P}$ - $\operatorname{dim} \mathcal{U}$, there is a constant $A(n)$ depending only on $n$ 
such that

$$
\left|u^{\mathcal{G}} \cap U\right| \geqslant \frac{2}{3}|U|
$$

for all $q \geqslant A(n)$ and all $\lambda \vdash n$. By taking $g(n)$ large enough, say

$$
g(n) \geqslant \max _{\substack{q^{\prime}=p^{r}<A(n) \\ 1 \neq w \in \operatorname{GL}_{n}\left(q^{\prime}\right) \\ w \text { is unipotent } \\ \psi \in \operatorname{IBr}_{\ell}\left(\operatorname{GL}_{n}\left(q^{\prime}\right)\right)}}\left\{\frac{|\psi(w)|}{\psi(1)^{(n-2) /(n-1)}}\right\},
$$

we may assume that the condition $q \geqslant A(n)$ is indeed satisfied.

Let $1 \neq v \in U \backslash u^{\mathcal{G}}$ be labeled by $\nu \vdash n$. Then,

$$
v \in \mathcal{U}=\overline{u^{\mathcal{G}} \cap \mathcal{U}}
$$

and so $v^{\mathcal{G}} \leqslant u^{\mathcal{G}}$. In particular, if $u^{\mathcal{G}}$ is minimal with respect to $\leqslant$, then no such $v$ exists. If $u^{\mathcal{G}}$ is not minimal, then, by the induction hypothesis applied to $v^{\mathcal{G}}$, we have

$$
|\varphi(v)| \leqslant g_{\nu}(n) \varphi(1)^{(n-2) /(n-1)}
$$

for some positive constant $g_{\nu}(n)$ depending only on $\nu$. We will let $g_{\lambda}^{\prime}(n)$ be the largest among all $g_{\nu}(n)$ when $\nu$ runs over the partitions for all such $v$.

Let $\varrho:={ }^{*} R_{L}^{G}(\varphi)$, where $L$ is a Levi subgroup of $P$. Then,

$$
\varrho(1)=\left[\varphi_{U}, 1_{U}\right]_{U}=\frac{1}{|U|}\left(\varphi(1)+\sum_{1 \neq v \in U \backslash u^{\mathcal{G}}} \varphi(v)+\sum_{w \in u^{\mathcal{G}} \cap U} \varphi(w)\right),
$$

and so

$$
\left|u^{\mathcal{G}} \cap U\right||\varphi(u)| \leqslant|U| \varrho(1)+\sum_{1 \neq v \in U \backslash u^{\mathcal{G}}}|\varphi(v)|+\varphi(1) .
$$

It now follows from (3.16) and (3.18) that

$$
|\varphi(u)| \leqslant \frac{3}{2} \varrho(1)+\frac{1}{2} g_{\lambda}^{\prime}(n) \varphi(1)^{(n-2) /(n-1)}+\frac{3}{2|U|} \varphi(1) .
$$

The proof of Theorem 1.4 and the bound $\alpha \leqslant(n-2) /(n-1)$ in Proposition 4.3 imply that

$$
\varrho(1) \leqslant h(n) \varphi(1)^{(n-2) /(n-1)} .
$$

On the other hand, $|U| \geqslant q^{n-1}$ and $\varphi(1)<q^{n^{2} / 2}$, whence for $n \geqslant 4$ we have

$$
\frac{\varphi(1)}{|U|}<\varphi(1)^{(n-2) /(n-1)} \text {. }
$$

The same conclusion holds for $n=3$, since $\varphi(1)<q^{4}$ in this case. Hence the statement follows for $u$ by taking

$$
g_{\lambda}(n):=\frac{3}{2} h(n)+\frac{1}{2} g_{\lambda}^{\prime}(n)+\frac{3}{2} .
$$




\subsection{Special linear groups}

Proposition 3.4. Let $n \geqslant 3$ and $\mathbb{F}$ be an algebraically closed field of characteristic $\ell$, where either $\ell=0$ or $\ell \nmid q$. Let $V$ be an irreducible $\mathbb{F G L}(q)$-module which is reducible over $\mathrm{SL}_{n}(q)$. Then, one of the following properties holds:

(i) $\operatorname{dim}(V)>q^{\left(n^{2}+n\right) / 4}(q-1)$;

(ii) $n=3$ and $\operatorname{dim}(V) \geqslant(q-1)\left(q^{2}-1\right)$;

(iii) $n=4$ and $\operatorname{dim}(V) \geqslant(q-1)\left(q^{2}-1\right)\left(q^{3}-1\right)$;

(iv) $2 \mid n$, and $\operatorname{dim}(V)=\prod_{j=1}^{n / 2}\left(q^{2 j-1}-1\right)$ or $\prod_{j=1}^{n / 2}\left(q^{n / 2+j}-1\right) /\left(q^{j}-1\right)$; furthermore, $V$ is as described in [23, Proposition 5.10 (ii) and (iii)], and $\left.V\right|_{\mathrm{SL}_{n}(q)}$ is a sum of two irreducible constituents.

Proof. Repeat the same proof of [23, Proposition 5.10], but for all $n$.

\subsection{Proof of Theorem 1.5}

(i) In this proof, let $G:=\mathrm{GL}_{n}(q)=\mathcal{G}^{F}$ in the notation of $\S 2$, and let $S:=[G, G]$. Write $g=$ $s u=u s$, with $s \in G$ semisimple and $u \in G$ unipotent. By Theorem 1.4 and Proposition 4.3, we may assume that there is no proper split Levi subgroup $\mathcal{L}$ of $\mathcal{G}$ such that $\mathbf{C}_{G}(s) \leqslant \mathcal{L}^{F}$; equivalently, $\mathbf{C}_{G}(s) \cong \mathrm{GL}_{n / k}\left(q^{k}\right)$ for some $k \mid n$. For a fixed $n$, by choosing $h(n)$ large enough (similarly to the choice (3.17) of $g(n)$ ), we may assume that $q \geqslant 227$. Hence, we are done by Theorems 3.2 (when $k>1$ ) and 3.3 (when $k=1$ ) if $H=G$.

From now on, we will assume that $H=S$, and let $\tilde{\chi} \in \operatorname{Irr}(G)$ be lying above $\chi$. Applying the result for $G$, we are done if $\chi=\left.\widetilde{\chi}\right|_{S}$. Hence, we may assume that $\left.\tilde{\chi}\right|_{S}$ is reducible, and so either

$$
\chi(1) \geqslant \frac{\widetilde{\chi}(1)}{[G: S]}>q^{\left(n^{2}+n\right) / 4},
$$

or case (iv) of Proposition 3.4 holds for a $\mathbb{C} G$-module $V$ affording $\tilde{\chi}$. Since

$$
\frac{1}{2} \prod_{j=1}^{n / 2}\left(q^{2 j-1}-1\right)>\frac{q^{n^{2} / 4}}{2}\left(1-\sum_{j=1}^{n / 2} q^{1-2 j}\right)>q^{\left(n^{2}-1\right) / 4}
$$

when $2 \mid n$ and $q \geqslant 227$, we now have that

$$
\chi(1)>q^{\left(n^{2}-1\right) / 4} .
$$

Assume in addition that $g^{S}=g^{G}$. By Clifford's theorem, we may write $\left.\widetilde{\chi}\right|_{S}=\sum_{i=1}^{t} \chi^{x_{i}}$ for some elements $x_{i} \in G$. Since $g^{S}=g^{G}, g^{x_{i}}$ is $S$-conjugate to $g$ and so $\chi^{x_{i}}(g)=\chi(g)$. It follows that

$$
\frac{|\chi(g)|}{\chi(1)}=\frac{1}{t \chi(1)}\left|\sum_{i=1}^{t} \chi^{x_{i}}(g)\right|=\frac{|\widetilde{\chi}(g)|}{\widetilde{\chi}(1)}
$$


and so we are done again. So, we may assume that $g^{S} \neq g^{G}$.

(ii) Here we consider the case $k>1$, and recall that $u$ is a unipotent element in $\mathbf{C}_{G}(s)=\mathrm{GL}_{n / k}\left(q^{k}\right)$. Note that $\operatorname{det}_{\mathbb{F}_{q^{k}}}$ maps $\mathbf{C}_{G}(s)$ onto $\mathbb{F}_{q^{k}}^{\times}$, and the norm map $\mathbb{F}_{q^{k}}^{\times} \rightarrow \mathbb{F}_{q}^{\times}$ is surjective. It follows that $s^{G}=s^{S}$. Hence, our assumption $g^{G} \neq g^{S}$ implies that $u \neq 1$. It is well known that the centralizer of any non-central element in $\operatorname{GL}_{m}(q)$ has order at most $q^{m^{2}-2 m+2}$. It follows that

$$
\left|\mathbf{C}_{G}(g)\right|=\left|\mathbf{C}_{\mathbf{C}_{G}(s)}(u)\right| \leqslant q^{n^{2} / k-2 n+2 k} \leqslant q^{n^{2} / 2-2 n+4},
$$

whence $|\chi(g)| \leqslant\left|\mathbf{C}_{G}(g)\right|^{1 / 2} \leqslant q^{n^{2} / 4-n+2}$. Together with (3.20), this implies that

$$
|\chi(g)|<\chi(1)^{1-1 / 2 n}
$$

(iii) Now we consider the case $k=1$, i.e. $s \in \mathbf{Z}(G)$, and prove the stronger bound that

$$
|\chi(g)| \leqslant h(n) \chi(1)^{(n-2) /(n-1)} .
$$

Without loss of generality, we may assume that $g=u$. Let $r_{i}$ denote the number of Jordan blocks of size $i$ in the Jordan canonical form of $u$ for each $i \geqslant 1$; in particular, $\sum_{i} i r_{i}=n$. It is easy to see that $g^{G}=g^{S}$ if $\operatorname{gcd}\left(i: r_{i} \geqslant 1\right)=1$. So, the assumption $g^{G} \neq g^{S}$ implies

$$
\operatorname{gcd}\left(i: r_{i} \geqslant 1\right)>1
$$

in particular, $r_{1}=0$. We claim (for $n \geqslant 5$ ) that either

$$
\left|\mathbf{C}_{G}(g)\right| \leqslant q^{\left(n^{2}-3 n+6\right) / 2},
$$

or $g$ has type $J_{2}^{n / 2}$ (i.e. $r_{2}=\frac{1}{2} n$ ). Indeed, by [32, Theorem 3.1] we have that $\left|\mathbf{C}_{G}(g)\right|<q^{N}$, where

$$
N:=\sum_{i} i r_{i}^{2}+2 \sum_{i<j} i r_{i} r_{j}
$$

Now, if $r_{2}=0$, then $3 N \leqslant\left(\sum_{i} i r_{i}\right)^{2}=n^{2}$, and so (3.23) holds for $n \geqslant 6$. If $r_{2}=0$ and $n=5$, then (3.22) implies that $r_{5}=1$, again yielding (3.23). Suppose now that $\frac{1}{2} n>r:=r_{2}>0$, whence $r_{3}=0$ by $(3.22)$ and $n-2 r=t:=\sum_{j \geqslant 4} j r_{j} \geqslant 4$. Then,

$$
N=2 r^{2}+4 r \sum_{j \geqslant 4} r_{j}+\sum_{j \geqslant 4} j r_{j}^{2}+2 \sum_{4 \leqslant j<j^{\prime}} j r_{j} r_{j^{\prime}} \leqslant 2 r^{2}+r t+\frac{t^{2}}{4} \leqslant \frac{n^{2}-4 n+8}{2} .
$$

In the case of $(3.23),|\chi(g)| \leqslant q^{\left(n^{2}-3 n+6\right) / 4}$, and so (3.21) holds because of $(3.20)$. 
It remains to consider the case $g=J_{2}^{n / 2}$. Let $W=\mathbb{F}_{q}^{n}=\left\langle e_{1}, \ldots, e_{n}\right\rangle_{\mathbb{F}_{q}}$ denote the natural module for $G$, with $g\left(e_{1}\right)=e_{1}$. Here, $\left|\mathbf{C}_{G}(g)\right|<q^{n^{2} / 2}$ by (3.24), whence

$$
|\chi(g)| \leqslant q^{n^{2} / 4}
$$

Suppose first that

$$
\chi(1)>q^{(n-1)(n-2) / 2} .
$$

If $n \geqslant 8$, then (3.25) and (3.26) immediately imply (3.21). In the remaining case, we have $n=6$. An application of Clifford's theorem to the normal subgroup $\mathrm{SL}_{6}(q) \mathbf{Z}\left(\mathrm{GL}_{6}(q)\right)$ of $\mathrm{GL}_{6}(q)$ yields $2 \leqslant \tilde{\chi}(1) / \chi(1) \leqslant 6$. In particular, in the case of Proposition 3.4 (iv) we have

$$
\chi(1) \leqslant \frac{\left(q^{4}-1\right)\left(q^{5}-1\right)\left(q^{6}-1\right)}{2(q-1)\left(q^{2}-1\right)\left(q^{3}-1\right)}<q^{10},
$$

contrary to (3.26). Thus, Proposition 3.4(i) must hold, whence

$$
\chi(1) \geqslant \frac{1}{6} q^{21 / 2}(q-1) \geqslant q^{45 / 4}
$$

(for $q \geqslant 1301$, which can be guaranteed by taking $h(6)$ large enough). The latter, together with (3.25), implies (3.21).

It remains to consider the case where (3.26) does not hold. Let $\chi$ be afforded by a $\mathbb{C} S$-module $V$, and let $P:=\operatorname{Stab}_{S}\left(\left\langle e_{1}\right\rangle_{\mathbb{F}_{q}}\right)=U L$. We decompose the $P$-module $V$ as $\mathbf{C}_{V}(U) \oplus[U, V]$, and let $\gamma$ (resp. $\delta$ ) denote the $P$-character of $\mathbf{C}_{V}(U)$ (resp. $\left.[U, V]\right)$. In particular, $\gamma={ }^{*} R_{L}^{S}(\chi)$, and so, arguing as in part (ii) of the proof of Theorem 1.4, we get

$$
|\gamma(g)| \leqslant \gamma(1) \leqslant f(n) \chi(1)^{(n-2) /(n-1)}
$$

(for some function $f: \mathbb{N} \rightarrow \mathbb{N}$ ). Next, we decompose

$$
[U, V]=\sum_{1_{U} \neq \lambda \in \operatorname{Irr}(U)} V_{\lambda}
$$

as a direct sum of $U$-eigenspaces, which are transitively permuted by $L \cong \mathrm{GL}_{n-1}(q)$. Note that $g$ has prime order $p \mid q$, and it acts on $\operatorname{Irr}(U) \backslash\left\{1_{U}\right\}$ with exactly $q^{n / 2}-1$ fixed points. Certainly, the trace of $g$ in its action on $\sum_{\lambda \in \mathcal{O}} V_{\lambda}$ for any non-trivial $g$-orbit $\mathcal{O}$ on $\operatorname{Irr}(U) \backslash\left\{1_{U}\right\}$ is zero. Since $\chi(1) \leqslant q^{(n-1)(n-2) / 2}$, we have that

$$
|\delta(g)| \leqslant\left(q^{n / 2}-1\right) \operatorname{dim}\left(V_{\lambda}\right)=\left(q^{n / 2}-1\right) \frac{\operatorname{dim}([U, V])}{q^{n-1}-1}<\frac{\chi(1)}{q^{n / 2-1}} \leqslant \chi(1)^{(n-2) /(n-1)} .
$$

Together with (3.27), this completes the proof. 
The above proof yields the following analogue of Theorem 3.3:

Corollary 3.5. Let $S:=\mathrm{SL}_{n}(q) \leqslant G:=\mathrm{GL}_{n}(q)$, and $u \in G$ be any non-trivial unipotent element. Assume that either $\ell=0$, or $\ell \nmid q$ and $u^{G}=u^{S}$. Then, for any $\varphi \in \operatorname{IBr}_{\ell}(S)$,

$$
|\varphi(u)| \leqslant g(n) \varphi(1)^{(n-2) /(n-1)} .
$$

Remark 3.6. For any $\varepsilon>0$, it seems possible to improve the term $q^{n / 3}$ in Theorem 3.2 to $q^{n /(2+\varepsilon)}$ at the price of using much bigger $f(n)$, as well as a much bigger lower bound on $q$. As a consequence, one could perhaps improve the exponent $1-1 / 2 n$ in Theorem 1.5 to $1-1 /(1+\varepsilon) n$. But we did not try to pursue it.

\section{Bounds for the constant $\alpha(\mathcal{L})$ : Proofs of Theorems $1.6,1.7$ and 1.10}

For the proof of Theorem 1.6, it is convenient to handle the classical types SL, Sp and SO separately. As in the theorem, let $\mathbb{K}$ be an algebraically closed field of good characteristic. Note that, by the defnition of $\alpha(\mathcal{L})$, this value does not depend on the isogeny type of $\mathcal{G}$.

\subsection{Case $\mathcal{G}=\mathrm{GL}_{n}(\mathbb{K})$ or $\mathcal{G}=\mathrm{SL}_{n}(\mathbb{K})$}

To prove Theorem 1.6, in this case we use the following lemma, which transfers attention from unipotent to semisimple elements in the analysis of $\alpha(\mathcal{L})$. Denote by $J_{i}$ a unipotent $i \times i$ Jordan block matrix, and by $\sum_{i} J_{i}^{n_{i}}$ the matrix in $\mathrm{SL}_{n}(\mathbb{K})$ with $n_{i}$ diagonal blocks $J_{i}$ for each $i$, where $n=\sum_{i} i n_{i}$.

LEMMA 4.1. Let $u=\sum_{i} J_{i}^{n_{i}}$ be a unipotent element of $\mathrm{GL}_{n}(\mathbb{K})$, where $n=\sum i n_{i}$. Let $\lambda_{j}, j \in \mathbb{N}$, be distinct scalars in $\mathbb{K}^{\times}$, and for each $i$ let $D_{i}=\operatorname{diag}\left(\lambda_{1}, \lambda_{2}, \ldots, \lambda_{i}\right)$. Define

$$
s:=\sum_{i} D_{i}^{n_{i}} \in \mathrm{GL}_{n}(\mathbb{K})
$$

Then, $\operatorname{dim} \mathbf{C}_{\mathrm{GL}_{n}(\mathbb{K})}(u)=\operatorname{dim} \mathbf{C}_{\mathrm{GL}_{n}(\mathbb{K})}(s)$.

Proof. Observe that

$$
\operatorname{dim} \mathbf{C}_{\mathrm{GL}_{n}(\mathbb{K})}(s)=\left(\sum_{i \geqslant 1} n_{i}\right)^{2}+\left(\sum_{i \geqslant 2} n_{i}\right)^{2}+\ldots=\sum_{i} i n_{i}^{2}+2 \sum_{i<j} i n_{i} n_{j}
$$

which is equal to $\operatorname{dim} \mathbf{C}_{\mathrm{GL}_{n}(\mathbb{K})}(u)$ by $[32$, Theorem 3.1].

For a subgroup $X$ of $\mathrm{GL}_{n}(\mathbb{K})$, define $X_{\mathrm{ss}}$ to be the set of semisimple elements of $X$. 
COROLlary 4.2. If $n \geqslant 2$ and $\mathcal{L}$ is a Levi subgroup of $\mathcal{G}=\mathrm{GL}_{n}(\mathbb{K})$ or $\mathcal{G}=\mathrm{SL}_{n}(\mathbb{K})$, then

$$
\alpha(\mathcal{L}) \leqslant \max _{s \in \mathcal{L}_{\mathrm{ss}} \backslash \mathbf{Z}(\mathcal{G})} \frac{\operatorname{dim} s^{\mathcal{L}}}{\operatorname{dim} s^{\mathcal{G}}} .
$$

Proof. We have

$$
\mathcal{L}=\mathcal{G} \cap \prod_{j=1}^{r} \mathrm{GL}_{a_{j}}(\mathbb{K}),
$$

where $\sum_{j=1}^{r} a_{j}=n$. Let $u \in \mathcal{L}_{\text {unip }}$, so that

$$
u=\sum_{j=1}^{r} \sum_{i} J_{i}^{n_{i j}},
$$

where $\sum_{i} i n_{i j}=a_{j}$. The condition $u \neq 1$ means that there are some $i \geqslant 2$ and $j \geqslant 1$ such that $n_{i j}>0$. If we define

$$
s=\sum_{j=1}^{r} \sum_{i} D_{i}^{n_{i j}},
$$

where $D_{i}$ is as in the statement of Lemma 4.1 (and the scalars $\lambda_{j}$ are chosen so that $s$ has determinant 1 in the case where $\left.\mathcal{G}=\mathrm{SL}_{n}(\mathbb{K})\right)$, then $s \notin \mathbf{Z}(\mathcal{G})$. Now, Lemma 4.1 shows that $\operatorname{dim} \mathbf{C}_{\mathcal{L}}(u)=\operatorname{dim} \mathbf{C}_{\mathcal{L}}(s)$ and $\operatorname{dim} \mathbf{C}_{\mathcal{G}}(u)=\operatorname{dim} \mathbf{C}_{\mathcal{G}}(s)$.

Proof of Theorem 1.6 for $\mathrm{GL}_{n}(\mathbb{K})$ and $\mathrm{SL}_{n}(\mathbb{K})$. We prove the result for $\mathcal{G}=\mathrm{GL}_{n}(\mathbb{K})$ and point out the small adjustment needed for $\mathrm{SL}_{n}(\mathbb{K})$ at the end of the proof. Let $\mathcal{L}$ be a Levi subgroup of $\mathcal{G}$. Adopting an obvious notational convention we take

$$
\mathcal{L}=\mathrm{GL}_{a}(\mathbb{K}) \times \mathrm{GL}_{b}(\mathbb{K}) \times \ldots \times \mathrm{GL}_{z}(\mathbb{K}) .
$$

Write $V_{n}=V_{a} \oplus V_{b} \oplus \ldots \oplus V_{z}$ for the corresponding direct sum decomposition of $V_{n}=\mathbb{K}^{n}$.

In view of Corollary 4.2 , it suffices to prove that

$$
\max _{s \in \mathcal{L}_{\mathrm{ss}} \backslash \mathbf{Z}(\mathcal{G})} \frac{\operatorname{dim} s^{\mathcal{L}}}{\operatorname{dim} s^{\mathcal{G}}} \leqslant \frac{1}{2}\left(1+\frac{\operatorname{dim} \mathcal{L}}{\operatorname{dim} \mathcal{G}}\right) .
$$

Let $s$ be a semisimple element of $\mathcal{L}$, and let $\lambda_{1}, \ldots, \lambda_{k}$ be the distinct eigenvalues of $s$ on $V_{n}$. Write

$$
\left.s\right|_{V_{a}}=\operatorname{diag}\left(\lambda_{1}^{\left(a_{1}\right)}, \ldots, \lambda_{k}^{\left(a_{k}\right)}\right), \quad \ldots,\left.\quad s\right|_{V_{z}}=\operatorname{diag}\left(\lambda_{1}^{\left(z_{1}\right)}, \ldots, \lambda_{k}^{\left(z_{k}\right)}\right),
$$

where $\sum_{i=1}^{k} a_{i}=a$, and so on (superscripts denote multiplicities). Then,

$$
\begin{aligned}
& \mathbf{C}_{\mathcal{G}}(s)=\mathrm{GL}_{a_{1}+b_{1}+\ldots}(\mathbb{K}) \times \ldots \times \mathrm{GL}_{a_{k}+b_{k}+\ldots}(\mathbb{K}), \\
& \mathbf{C}_{\mathcal{L}}(s)=\prod_{i=1}^{k} \mathrm{GL}_{a_{i}}(\mathbb{K}) \times \prod_{i=1}^{k} \mathrm{GL}_{b_{i}}(\mathbb{K}) \times \ldots
\end{aligned}
$$


To prove (4.1), we need to show

$$
\frac{1}{2} \frac{\operatorname{dim} \mathcal{G}-\operatorname{dim} \mathcal{L}}{\operatorname{dim} \mathcal{G}} \leqslant \frac{\operatorname{dim} s^{\mathcal{G}}-\operatorname{dim} s^{\mathcal{L}}}{\operatorname{dim} s^{\mathcal{G}}}
$$

Now

$$
\frac{1}{2} \frac{\operatorname{dim} \mathcal{G}-\operatorname{dim} \mathcal{L}}{\operatorname{dim} \mathcal{G}}=\frac{a b+a c+b c+\ldots}{(a+b+\ldots)^{2}}
$$

while

$$
\frac{\operatorname{dim} s^{\mathcal{G}}-\operatorname{dim} s^{\mathcal{L}}}{\operatorname{dim} s^{\mathcal{G}}}=\frac{\sum_{i \neq j}\left(a_{i} b_{j}+a_{i} c_{j}+b_{i} c_{j}+\ldots\right)}{\sum_{i<j}\left(a_{i}+b_{i}+\ldots\right)\left(a_{j}+b_{j}+\ldots\right)} .
$$

Hence, (4.2) is equivalent to the inequality

$$
\begin{gathered}
\left(\left(\sum_{i} a_{i}\right)\left(\sum_{i} b_{i}\right)+\left(\sum_{i} a_{i}\right)\left(\sum_{i} c_{i}\right)+\left(\sum_{i} b_{i}\right)\left(\sum_{i} c_{i}\right)+\ldots\right) \\
\times\left(\sum_{i<j}\left(a_{i}+b_{i}+\ldots\right)\left(a_{j}+b_{j}+\ldots\right)\right) \\
\leqslant\left(\sum_{i} a_{i}+\sum_{i} b_{i}+\ldots\right)^{2} \sum_{i \neq j}\left(a_{i} b_{j}+a_{i} c_{j}+b_{i} c_{j}+\ldots\right) .
\end{gathered}
$$

Now, observe that all the terms on the left-hand side of this inequality appear with at most the same multiplicity on the right-hand side. Hence (4.3) holds, and the proof is complete for $\mathcal{G}=\mathrm{GL}_{n}(\mathbb{K})$.

For the case where $\mathcal{G}=\mathrm{SL}_{n}(\mathbb{K})$, we need to prove inequality (4.3) with the first term on the right-hand side replaced by $\left(\sum_{i} a_{i}+\sum_{i} b_{i}+\ldots\right)^{2}-1$. This remains true, since the terms in the right-hand side that are not in the left-hand side of (4.3) include $\sum_{i \neq j}\left(a_{i}^{3} b_{j}+a_{i}^{3} c_{j}+b_{i}^{3} c_{j}+\ldots\right)$, which is at least $\sum_{i \neq j}\left(a_{i} b_{j}+a_{i} c_{j}+b_{i} c_{j}+\ldots\right) \geqslant 1$.

We also deduce the following general bound, which was used in the proof of Theorem 3.3, and also in Example 2.8.

Proposition 4.3. If $\mathcal{L}$ is a Levi subgroup of $\mathcal{G}=\mathrm{GL}_{n}(\mathbb{K})$, then

$$
\alpha(\mathcal{L}) \leqslant \frac{n-2}{n-1}
$$

with equality if and only if $\mathcal{L}=\mathrm{GL}_{n-1}(\mathbb{K}) \times \mathrm{GL}_{1}(\mathbb{K})$.

Proof. Choose maximal $a \leqslant \frac{1}{2} n$ such that $\mathcal{L} \leqslant \mathrm{GL}_{a}(\mathbb{K}) \times \mathrm{GL}_{n-a}(\mathbb{K})$. By Theorem 1.6 for $\mathrm{GL}_{n}(\mathbb{K})$, proved above, we have

$$
\alpha(\mathcal{L}) \leqslant \frac{1}{2}\left(1+\frac{\operatorname{dim} \mathcal{L}}{\operatorname{dim} \mathcal{G}}\right) \leqslant \frac{1}{2}\left(1+\frac{a^{2}+(n-a)^{2}}{n^{2}}\right) .
$$


One checks that the right-hand side is less than $(n-2) /(n-1)$ for $n \geqslant 2 a$, except in the following cases:

(a) $a=1$, in which case $\mathcal{L}=\mathrm{GL}_{1}(\mathbb{K}) \times \mathrm{GL}_{n-1}(\mathbb{K})$ (by the maximal choice of $a$ );

(b) $a=2$ and $n \leqslant 5$.

In case (b) we compute the values of $\alpha(\mathcal{L})$ and find that $\alpha(\mathcal{L}) \leqslant \frac{1}{2}<(n-2) /(n-1)$ (note that $n \geqslant 2 a=4$ in this case).

It remains to consider case (a). We claim that, in this case, $\alpha(\mathcal{L})=(n-2) /(n-1)$. Let $u$ be a non-trivial unipotent element of $\mathcal{L}$, and write $u=\sum_{i} J_{i}^{n_{i}}$, where $\sum_{i} i n_{i}=n$. Then, $u$ projects to the element $J_{1}^{n_{1}-1}+\sum_{i \geqslant 2} J_{i}^{n_{i}}$ in the factor $\mathrm{GL}_{n-1}(\mathbb{K})$ of $\mathcal{L}$, and so, by [32, Theorem 3.1], we have

$$
\begin{aligned}
\operatorname{dim} \mathbf{C}_{\mathcal{G}}(u) & =\sum_{i} i n_{i}^{2}+2 \sum_{i<j} i n_{i} n_{j}, \\
\operatorname{dim} \mathbf{C}_{\mathcal{L}}(u) & =1+\left(n_{1}-1\right)^{2}+\sum_{i \geqslant 2} i n_{i}^{2}+2\left(n_{1}-1\right) \sum_{j \geqslant 2} n_{j}+2 \sum_{2 \leqslant i<j} i n_{i} n_{j} .
\end{aligned}
$$

Defining $s:=\operatorname{dim}[V, u]=n-\sum n_{i}$, it follows that

$$
(\operatorname{dim} \mathcal{G}-\operatorname{dim} \mathcal{L})-\left(\operatorname{dim} \mathbf{C}_{\mathcal{G}}(u)-\operatorname{dim} \mathbf{C}_{\mathcal{L}}(u)\right)=2 s .
$$

Next, observe that

$$
\begin{aligned}
\frac{\operatorname{dim} u^{\mathcal{L}}}{\operatorname{dim} u^{\mathcal{G}}} \leqslant \frac{n-2}{n-1} & \Longleftrightarrow(n-1)\left((\operatorname{dim} \mathcal{G}-\operatorname{dim} \mathcal{L})-\left(\operatorname{dim} \mathbf{C}_{\mathcal{G}}(u)-\operatorname{dim} \mathbf{C}_{\mathcal{L}}(u)\right)\right) \geqslant \operatorname{dim} u^{\mathcal{G}} \\
& \Longleftrightarrow 2(n-1) s \geqslant \operatorname{dim} u^{\mathcal{G}} .
\end{aligned}
$$

By [33, Lemma 3.4(i)] and its proof, we have $\operatorname{dim} u^{\mathcal{G}} \leqslant s(2 n-s)$, and so the above inequality holds when $s \geqslant 2$. Finally, when $s=1$ we have $u=J_{2}+J_{1}^{n-2}$, and we calculate that

$$
\frac{\operatorname{dim} u^{\mathcal{L}}}{\operatorname{dim} u^{\mathcal{G}}}=\frac{n-2}{n-1} .
$$

Hence, $\alpha(\mathcal{L})=(n-2) /(n-1)$ in case (a), and the proof is complete.

\subsection{Symplectic groups}

Now, we prove Theorem 1.6 for symplectic groups. We revert to Lie-theoretic notation, so assume that

$$
\mathcal{G}=C_{n}=\operatorname{Sp}_{2 n}(\mathbb{K})=\operatorname{Sp}(V),
$$

where $V=V_{2 n}(K)$ is the natural module for $\mathcal{G}$ and $n \geqslant 2$. 
Let $\mathcal{L}$ be a Levi subgroup of $\mathcal{G}$, so that

$$
\mathcal{L}^{\prime}=C_{n-r} \times \prod A_{r_{i}} \leqslant C_{n-r} \times A_{r-1},
$$

where $1 \leqslant r \leqslant n$. The first lemma deals with the case where $r=n$ (recall that $T_{r}$ denotes an $r$-dimensional torus).

LEMMA 4.4. If $\mathcal{L} \leqslant A_{n-1} T_{1}$, then $\alpha(\mathcal{L}) \leqslant \frac{1}{2}$.

Proof. Assume that $\mathcal{L} \leqslant A_{n-1} T_{1}=\mathrm{GL}_{n}(\mathbb{K})$, and let $u$ be a non-trivial unipotent element of $\mathcal{L}$. Write $u=\sum_{i} J_{i}^{n_{i}} \in \mathrm{SL}_{n}(q)$, where $\sum_{i} i n_{i}=n$. As an element of $G=\operatorname{Sp}_{2 n}(q), u$ has Jordan form $\sum_{i} J_{i}^{2 n_{i}}$. Hence, by [32, Theorem 3.1],

$$
\begin{aligned}
\operatorname{dim} \mathbf{C}_{\mathrm{GL}_{n}(\mathbb{K})}(u) & =\sum_{i} i n_{i}^{2}+2 \sum_{i<j} i n_{i} n_{j}=: c_{u}, \\
\operatorname{dim} \mathbf{C}_{\mathcal{G}}(u) & =2 \sum_{i} i n_{i}^{2}+4 \sum_{i<j} i n_{i} n_{j}+\sum_{i \text { odd }} n_{i} .
\end{aligned}
$$

So, $\operatorname{dim} \mathbf{C}_{\mathcal{G}}(u)=2 c_{u}+s_{u}$, where $s_{u}=\sum_{i \text { odd }} n_{i}$. It follows that

$$
\frac{\operatorname{dim} u^{\mathcal{L}}}{\operatorname{dim} u^{\mathcal{G}}} \leqslant \frac{\operatorname{dim} u^{\mathrm{GL}_{n}}}{\operatorname{dim} u^{\mathcal{G}}} \leqslant \frac{n^{2}-c_{u}}{2 n^{2}+n-2 c_{u}-s_{u}} \leqslant \frac{1}{2},
$$

and the conclusion follows.

Lemma 4.5. If $\mathcal{L}=C_{n-r} T_{r}$, then

$$
\alpha(\mathcal{L}) \leqslant \frac{1}{2}\left(1+\frac{\operatorname{dim} \mathcal{L}}{\operatorname{dim} \mathcal{G}}\right) .
$$

Proof. Let $u$ be a non-trivial unipotent element of $\mathcal{L}^{\prime}=C_{n-r}=\operatorname{Sp}_{2 n-2 r}(\mathbb{K})$, and write $u=\sum_{i} J_{i}^{n_{i}}$ with $\sum_{i} i n_{i}=2 n-2 r$. In $G=\operatorname{Sp}_{2 n}(q), u$ has Jordan form $J_{1}^{n_{1}+2 r}+\sum_{i \geqslant 2} J_{i}^{n_{i}}$. Using [32, Theorem 3.1], we find that

$$
\operatorname{dim} \mathbf{C}_{\mathcal{L}}(u)-\operatorname{dim} \mathbf{C}_{\mathcal{G}}(u)=2 r \sum n_{i}+2 r^{2}
$$

As in [33, p. 509], define

$$
s:=\operatorname{dim}[V, u]=2 n-2 r-\sum n_{i} .
$$

Then, (4.4) implies that $\operatorname{dim} u^{\mathcal{G}}-\operatorname{dim} u^{\mathcal{L}}=2 r s$. It also follows from [33, Lemma 3.4] and its proof that

$$
\operatorname{dim} u^{\mathcal{G}} \leqslant \frac{1}{2} s(4 n-s+1)
$$

Now, observe that

$$
\begin{aligned}
\frac{\operatorname{dim} u^{\mathcal{L}}}{\operatorname{dim} u^{\mathcal{G}}} \leqslant \frac{1}{2}\left(1+\frac{\operatorname{dim} \mathcal{L}}{\operatorname{dim} \mathcal{G}}\right) & \Longleftrightarrow \operatorname{dim} u^{\mathcal{G}} \leqslant \frac{2 \operatorname{dim} \mathcal{G}\left(\operatorname{dim} u^{\mathcal{G}}-\operatorname{dim} u^{\mathcal{L}}\right)}{\operatorname{dim} \mathcal{G}-\operatorname{dim} \mathcal{L}} \\
& \Longleftrightarrow \operatorname{dim} u^{\mathcal{G}} \leqslant \frac{2 s\left(2 n^{2}+n\right)}{2 n-r} .
\end{aligned}
$$

Clearly (4.5) implies that the last inequality holds, and so we are done. 
LEMmA 4.6. Suppose that $\mathcal{L}^{\prime} \leqslant C_{n-r} \times A_{r-1} \leqslant C_{n-r} \times C_{r}<\mathcal{G}$, with $r>1$, and let $u=$ $u_{1} u_{2} \in \mathcal{L}$ be a unipotent element, with $u_{1} \in C_{n-r}, u_{2} \in A_{r-1}<C_{r}$. Then,

$$
\operatorname{dim} u^{\mathcal{G}} \geqslant \operatorname{dim} u_{1}^{\mathcal{G}}+\operatorname{dim} u_{2}^{C_{r}} .
$$

Proof. Let

$$
u_{1}=\sum_{i} J_{i}^{a_{i}} \in C_{n-r} \quad \text { and } \quad u_{2}=\sum_{i} J_{i}^{b_{i}} \in A_{r-1}
$$

where $\sum_{i} i a_{i}=2 n-2 r$ and $\sum_{i} i b_{i}=r$. Then,

$$
\begin{aligned}
u_{1} & =J_{1}^{a_{1}+2 r}+\sum_{i \geqslant 2} J_{i}^{a_{i}} \in \mathcal{G}=\operatorname{Sp}_{2 n}(\mathbb{K}), \\
u_{2} & =\sum_{i} J_{i}^{2 b_{i}} \in C_{r} \\
u & =\sum_{i} J_{i}^{a_{i}+2 b_{i}} \in \mathcal{G} .
\end{aligned}
$$

Now, using [32, Theorem 3.1], we compute that

$$
\begin{aligned}
\operatorname{dim} & \mathbf{C}_{\mathcal{G}}\left(u_{1}\right)+\operatorname{dim} \mathbf{C}_{C_{r}}\left(u_{2}\right)-\operatorname{dim} \mathbf{C}_{\mathcal{G}}(u) \\
& =2 r^{2}+r+2 r \sum_{i} a_{i}-2 \sum_{i} i a_{i} b_{i}-2 \sum_{i<j} i\left(a_{i} b_{j}+a_{j} b_{i}\right) \\
& =2 r^{2}+r+2\left(\left(\sum_{i} a_{i}\right)\left(\sum_{i} i b_{i}\right)-\sum_{i} i a_{i} b_{i}-\sum_{i<j} i\left(a_{i} b_{j}+a_{j} b_{i}\right)\right) \\
\geqslant & 2 r^{2}+r=\operatorname{dim} C_{r}
\end{aligned}
$$

and the result follows.

Proof of Theorem 1.6 for $\mathcal{G}=C_{n}$. Let $\mathcal{L}$ be a Levi subgroup of $\mathcal{G}$, so

$$
\mathcal{L}^{\prime}=C_{n-r} \times \prod A_{r_{i}} \leqslant C_{n-r} \times A_{r-1} \leqslant C_{n-r} \times C_{r},
$$

where $1 \leqslant r \leqslant n$. Let $u=u_{1} u_{2}$ be a non-trivial unipotent element of $\mathcal{L}$, where $u_{1} \in C_{n-r}$ and $u_{2} \in A_{r-1}$. Using Lemma 4.6, we have

$$
\frac{\operatorname{dim} u^{\mathcal{L}}}{\operatorname{dim} u^{\mathcal{G}}} \leqslant \frac{\operatorname{dim} u_{1}^{C_{n-r}}+\operatorname{dim} u_{2}^{A_{r-1}}}{\operatorname{dim} u_{1}^{\mathcal{G}}+\operatorname{dim} u_{2}^{C_{r}}} .
$$

Also Lemmas 4.4 and 4.5 imply that

$$
\frac{\operatorname{dim} u^{A_{r-1}}}{\operatorname{dim} u^{C_{r}}} \leqslant \frac{1}{2} \quad \text { and } \quad \frac{\operatorname{dim} u^{C_{n-r}}}{\operatorname{dim} u^{\mathcal{G}}} \leqslant \frac{1}{2}\left(1+\frac{\operatorname{dim} C_{n-r} T_{r}}{\operatorname{dim} \mathcal{G}}\right) .
$$

Hence, (4.6) implies that

$$
\frac{\operatorname{dim} u^{\mathcal{L}}}{\operatorname{dim} u^{\mathcal{G}}} \leqslant \frac{1}{2}\left(1+\frac{\operatorname{dim} C_{n-r} T_{r}}{\operatorname{dim} \mathcal{G}}\right) \leqslant \frac{1}{2}\left(1+\frac{\operatorname{dim} \mathcal{L}}{\operatorname{dim} \mathcal{G}}\right) .
$$

This completes the proof of Theorem 1.6 for $\mathcal{G}=C_{n}$. 


\subsection{Orthogonal groups}

We complete the proof of Theorem 1.6 by handling the orthogonal groups. The proof for $\mathcal{G}=B_{n}=\mathrm{SO}_{2 n+1}(\mathbb{K})$ is very similar to that for $\mathcal{G}=C_{n}$ : one shows that Lemmas 4.4-4.6 also hold in the $B_{n}$ case (with $\mathcal{L}=B_{n-r} T_{r}$ in Lemma 4.5 and $\mathcal{L}^{\prime} \leqslant B_{n-r} \times A_{r-1} \leqslant B_{n-r} \times D_{r}$ in Lemma 4.6), and the theorem follows. Things are a little different in the $D_{n}$ case, so assume now that

$$
\mathcal{G}=D_{n}=\mathrm{SO}_{2 n}(\mathbb{K})=\mathrm{SO}(V), \quad n \geqslant 4
$$

Let $\mathcal{L}$ be a Levi subgroup of $\mathcal{G}$. Then,

$$
\mathcal{L}^{\prime}=D_{n-r} \times \prod_{i} A_{r_{i}} \leqslant D_{n-r} \times A_{r-1},
$$

where $1 \leqslant r \leqslant n$ and $r \neq n-1$.

Lemma 4.7. Suppose $\mathcal{L}^{\prime} \leqslant A_{n-1}$ and $\mathcal{L}^{\prime} \neq A_{n-1}$. Then, $\alpha(\mathcal{L}) \leqslant \frac{1}{2}$.

Proof. By assumption, we have $\mathcal{L} \leqslant \mathrm{GL}_{a}(\mathbb{K}) \times \mathrm{GL}_{b}(\mathbb{K})$, where $a+b=n$ and $a, b \geqslant 1$. Let $u=u_{1} u_{2} \in \mathcal{L}$, where $u_{1}=\sum_{i} J_{i}^{a_{i}} \in \mathrm{GL}_{a}(\mathbb{K})$ and $u_{1}=\sum_{i} J_{i}^{b_{i}} \in \mathrm{GL}_{b}(\mathbb{K})$ (so $\sum_{i} i a_{i}=a$ and $\left.\sum_{i} i b_{i}=b\right)$. Then, $u=\sum_{i} J_{i}^{2 a_{i}+2 b_{i}} \in \mathcal{G}$. By [32, Theorem 3.1],

$$
\operatorname{dim} \mathbf{C}_{\mathcal{L}}(u)=\sum_{i} i a_{i}^{2}+\sum_{i} i b_{i}^{2}+2 \sum_{i<j} i a_{i} a_{j}+2 \sum_{i<j} i b_{i} b_{j}=: c_{u},
$$

and

$$
\begin{aligned}
\operatorname{dim} \mathbf{C}_{\mathcal{G}}(u) & =2 \sum_{i} i\left(a_{i}+b_{i}\right)^{2}+4 \sum_{i<j} i\left(a_{i}+b_{i}\right)\left(a_{j}+b_{j}\right)-\sum_{i \text { odd }}\left(a_{i}+b_{i}\right) \\
& =2 c_{u}+4 \sum_{i} i a_{i} b_{i}+4 \sum_{i<j} i\left(a_{i} b_{j}+a_{j} b_{i}\right)-\sum_{i \text { odd }}\left(a_{i}+b_{i}\right) .
\end{aligned}
$$

Then, $\operatorname{dim} u^{\mathcal{L}}=a^{2}+b^{2}-c_{u}$, while

$$
\operatorname{dim} u^{\mathcal{G}}=2(a+b)^{2}-(a+b)-\left(2 c_{u}+4 \sum_{i} i a_{i} b_{i}+4 \sum_{i<j} i\left(a_{i} b_{j}+a_{j} b_{i}\right)-\sum_{i \text { odd }}\left(a_{i}+b_{i}\right)\right) .
$$

To prove the lemma, we need to show that $\operatorname{dim} u^{\mathcal{G}} \geqslant 2\left(a^{2}+b^{2}-c_{u}\right)$. Using the equations $\sum_{i} i a_{i}=a$ and $\sum_{i} i b_{i}=b$, this amounts to showing that

$$
4 \sum_{i}\left(i^{2}-i\right) a_{i} b_{i}+4 \sum_{i<j} i(j-1)\left(a_{i} b_{j}+a_{j} b_{i}\right)+\sum_{i \text { odd }}\left(a_{i}+b_{i}\right) \geqslant \sum i a_{i}+\sum j b_{j} .
$$

Consider a term $k a_{k}+l b_{l}$ on the right-hand side, with $a_{k}, b_{l} \neq 0$. If $k=l=1$, this occurs in the sum $\sum_{i \text { odd }}\left(a_{i}+b_{i}\right)$; if $k=l \geqslant 2$, it is less than or equal to the term $4\left(k^{2}-k\right) a_{k} b_{k}$ on the left-hand side; and if $k<l$ or $l<k$, it is at most $4 k(l-1) a_{k} b_{l}$ or $4 l(k-1) a_{k} b_{l}$, respectively. Hence, the inequality (4.7) holds, completing the proof of the lemma. 
The proofs of the next two lemmas are very similar to those of Lemmas 4.5 and 4.6.

LEMMA 4.8. If $\mathcal{L}=D_{n-r} T_{r}$, then

$$
\alpha(\mathcal{L}) \leqslant \frac{1}{2}\left(1+\frac{\operatorname{dim} \mathcal{L}}{\operatorname{dim} \mathcal{G}}\right) .
$$

LEMmA 4.9. Suppose that $\mathcal{L}^{\prime} \leqslant D_{n-r} \times A_{r-1} \leqslant D_{n-r} \times D_{r}<\mathcal{G}$ with $r>1$, and let $u=$ $u_{1} u_{2} \in \mathcal{L}$ be a unipotent element, with $u_{1} \in D_{n-r}$ and $u_{2} \in A_{r-1}<D_{r}$. Then,

$$
\operatorname{dim} u^{\mathcal{G}} \geqslant \operatorname{dim} u_{1}^{\mathcal{G}}+\operatorname{dim} u_{2}^{D_{r}}
$$

Let $\mathcal{L}^{\prime}=D_{n-r} \times \mathcal{L}_{1} \leqslant D_{n-r} \times A_{r-1}$, where $\mathcal{L}_{1}=\prod_{i} A_{r_{i}} \leqslant A_{r-1}$. If either $\mathcal{L}_{1}<A_{r-1}$ or $r=1$, then Theorem 1.6 follows from Lemmas $4.7-4.9$ just as in the argument following (4.6) for the case where $\mathcal{G}=C_{n}$. It remains to handle the case where $\mathcal{L}^{\prime}=D_{n-r} \times A_{r-1}$ with $2 \leqslant r \leqslant n, r \neq n-1$. We deal with this case in the next two lemmas.

Lemma 4.10. Suppose $\mathcal{L}^{\prime}=D_{n-r} \times A_{r-1}$ with $r \geqslant 3$. Then,

$$
\alpha(\mathcal{L}) \leqslant \frac{1}{2}\left(1+\frac{\operatorname{dim} \mathcal{L}}{\operatorname{dim} \mathcal{G}}\right) .
$$

Proof. Let $u=u_{1} u_{2}$ be a unipotent element of $\mathcal{L}$, where $u_{1} \in D_{n-r}$ and $u_{2} \in A_{r-1}<D_{r}$. We will show that

$$
\frac{\operatorname{dim} u_{2}^{A_{r-1}}}{\operatorname{dim} u_{2}^{D_{r}}} \leqslant \frac{1}{2}\left(1+\frac{\operatorname{dim} \mathcal{L}}{\operatorname{dim} \mathcal{G}}\right) .
$$

Given this, the lemma follows, since by Lemma 4.9 we have

$$
\frac{\operatorname{dim} u^{\mathcal{L}}}{\operatorname{dim} u^{\mathcal{G}}} \leqslant \frac{\operatorname{dim} u_{1}^{D_{n-r}}+\operatorname{dim} u_{2}^{A_{r-1}}}{\operatorname{dim} u_{1}^{\mathcal{G}}+\operatorname{dim} u_{2}^{D_{r}}},
$$

and this is at most $\frac{1}{2}(1+\operatorname{dim} \mathcal{L} / \operatorname{dim} \mathcal{G})$, by Lemma 4.8 and (4.8).

It remains to establish (4.8). Let $u_{2}=\sum J_{i}^{a_{i}} \in A_{r-1}=\mathrm{SL}_{r}(\mathbb{K})$, so that $u_{2}$ has Jordan form $\sum J_{i}^{2 a_{i}}$ in $D_{r}$. Then,

$$
\begin{aligned}
\operatorname{dim} \mathbf{C}_{\mathrm{GL}_{r}(\mathbb{K})}\left(u_{2}\right) & =\sum_{i} i a_{i}^{2}+2 \sum_{i<j} i a_{i} a_{j}, \\
\operatorname{dim} \mathbf{C}_{D_{r}}\left(u_{2}\right) & =2 \sum_{i} i a_{i}^{2}+4 \sum_{i<j} i a_{i} a_{j}-\sum_{i \text { odd }} a_{i} .
\end{aligned}
$$

Write $s_{2}:=\sum_{i \text { odd }} a_{i}$. Then,

$$
\frac{\operatorname{dim} u_{2}^{A_{r-1}}}{\operatorname{dim} u_{2}^{D_{r}}}=\frac{1}{2}\left(1+\frac{r-s_{2}}{\operatorname{dim} u_{2}^{D_{r}}}\right) .
$$




\begin{tabular}{|c|c|c|c|c|c|c|}
\hline$u_{2} \in \mathrm{SL}_{5}(q)$ & $J_{2}+J_{1}^{3}$ & $J_{2}^{2}+J_{1}$ & $J_{3}+J_{1}^{2}$ & $J_{3}+J_{2}$ & $J_{4}+J_{1}$ & $J_{5}$ \\
\hline $\operatorname{dim} u_{2}^{D_{5}}$ & 14 & 20 & 26 & 28 & 32 & 36 \\
\hline$s_{2}$ & 3 & 1 & 3 & 1 & 1 & 1 \\
\hline
\end{tabular}

Table 2 .

Therefore, to prove (4.8) it suffices to show that

$$
\frac{r-s_{2}}{\operatorname{dim} u_{2}^{D_{r}}} \leqslant \frac{\operatorname{dim} \mathcal{L}}{\operatorname{dim} \mathcal{G}}=\frac{2(n-r)^{2}-(n-r)+r^{2}}{2 n^{2}-n} .
$$

It is straightforward to see that the right-hand side of (4.9) is at least $\frac{1}{3}$, so (4.9) holds if $\operatorname{dim} u_{2}^{D_{r}} \geqslant 3\left(r-s_{2}\right)$. The minimum value of $\operatorname{dim} u_{2}^{D_{r}}$ occurs when $u_{2}=J_{2}+J_{1}^{r-2} \in \mathrm{SL}_{r}(q)$, in which case $\operatorname{dim} u_{2}^{D_{r}}=4 r-6$. This shows that (4.9) holds when $r \geqslant 6$.

It remains to establish (4.9) for $r=3,4,5$. For $r=5$, the possibilities for $u_{2} \in \mathrm{SL}_{r}(q)$ are listed in Table 2 above. For all these possibilities (4.9) holds. The arguments for $r=3,4$ are similar.

Lemma 4.11. Suppose $\mathcal{L}^{\prime}=D_{n-2} \times A_{1}$. Then,

$$
\alpha(\mathcal{L}) \leqslant \frac{1}{2}\left(1+\frac{\operatorname{dim} \mathcal{L}}{\operatorname{dim} \mathcal{G}}\right) .
$$

Proof. Let $u=u_{1} u_{2} \in \mathcal{L}$, with $u_{1}=\sum_{i} J_{i}^{n_{i}} \in D_{n-2} \quad\left(\right.$ so $\left.\sum_{i} i n_{i}=2 n-4\right)$ and $u_{2} \in A_{1}$. If $u_{2}=1$, then the conclusion follows from Lemma 4.8, so assume that $u_{2} \neq 1$. Then, $\operatorname{dim} u_{2}^{A_{1}}=2$ and

$$
u=J_{2}^{n_{2}+2}+\sum_{i \neq 2} J_{i}^{n_{i}} \in \mathcal{G}=D_{n}
$$

By [32, Theorem 3.1],

$$
\begin{aligned}
\operatorname{dim} \mathbf{C}_{\mathcal{L}}(u) & =\frac{1}{2} \sum i n_{i}^{2}+\sum_{i<j} i n_{i} n_{j}-\frac{1}{2} \sum_{i \text { odd }} n_{i}+2, \\
\operatorname{dim} \mathbf{C}_{\mathcal{G}}(u) & =\frac{1}{2} \sum i n_{i}^{2}+\sum_{i<j} i n_{i} n_{j}-\frac{1}{2} \sum_{i \text { odd }} n_{i}+2 n_{1}+4 \sum_{i \geqslant 2} n_{i}+4 .
\end{aligned}
$$

Define $s:=\operatorname{dim}[V, u]=2 n-\sum n_{i}-2$. Then,

$$
\operatorname{dim} u^{\mathcal{G}}-\operatorname{dim} u^{\mathcal{L}}=4 s+2 n_{1}-8 .
$$

Also, [33, Lemma 3.4] gives $\operatorname{dim} u^{\mathcal{G}} \leqslant \frac{1}{2} s(4 n-s+1)$. Hence, we see that the desired inequality

$$
\frac{\operatorname{dim} u^{\mathcal{L}}}{\operatorname{dim} u^{\mathcal{G}}} \leqslant \frac{1}{2}\left(1+\frac{\operatorname{dim} \mathcal{L}}{\operatorname{dim} \mathcal{G}}\right)
$$


is equivalent to the following:

$$
\frac{1}{2} s(4 n-s+1) \leqslant \frac{\left(2 n^{2}-n\right)\left(4 s+2 n_{1}-8\right)}{4 n-7} .
$$

Now, $2 n-4=\sum_{i} i n_{i} \geqslant 2 \sum_{i \geqslant 2} n_{i}$, and hence

$$
s=2 n-2-n_{1}-\sum_{i \geqslant 2} n_{i} \geqslant 2 n-2-n_{1}-(n-2)=n-n_{1} .
$$

It follows that $4 s+2 n_{1} \geqslant 2 n+2 s$, and hence (4.10) holds provided

$$
\frac{1}{2} s(4 n-s+1) \leqslant \frac{\left(2 n^{2}-n\right)(2 n+2 s-8)}{4 n-7},
$$

which is true for all $s$ when $n \geqslant 5$. Finally, when $n=4$, the conclusion of the lemma is easily checked directly. This completes the proof.

This completes the proof of Theorem 1.6.

\subsection{Exceptional groups: Proof of Theorem 1.7}

Let $\mathcal{G}$ be a simple algebraic group of exceptional type in good characteristic. In principle, one can check Theorem 1.7 by going through all possible Levi subgroups $\mathcal{L}$ of $\mathcal{G}$, in each case listing all the unipotent class representatives $u$ in $\mathcal{L}$ and using Theorem 3.1 and Tables 22.1.1-5 of [32] to write down the dimensions of $u^{\mathcal{L}}$ and $u^{\mathcal{G}}$. In fact, this is precisely what we do for the Levi subgroups listed in Table 1, and for the remaining ones (labelled "rest" in Table 1) we need a short argument.

We will give the proof of Theorem 1.7 just for $\mathcal{G}=E_{7}$, and leave the other entirely similar cases to the reader. First, suppose that the Levi subgroup $\mathcal{L}$ is one of those listed for $\mathcal{G}=E_{7}$ in Table 1 . In each case, we adopt the above procedure of listing unipotent representatives $u$ in $\mathcal{L}$ and calculating $\operatorname{dim} u^{\mathcal{L}}$ and $\operatorname{dim} u^{\mathcal{G}}$. We illustrate in Table 3 the case $\mathcal{L}^{\prime}=D_{6}$, listing in the first row the Jordan form of $u$ on the 12-dimensional module for $\mathcal{L}^{\prime}$ and in the second row the class of $u$ in $\mathcal{G}$ as in [32, Table 22.1.2].

To compute the information in the tables, we list the possible Jordan forms for unipotent elements $u$ of $D_{6}$, and in each case find a Levi subgroup of $D_{6}$ in which $u$ is contained as a regular element; this Levi subgroup then gives the label of $u$ as an element of $E_{7}$ in [32, Table 22.1.2]. For cases where all the Jordan blocks have even size - namely the Jordan forms $\left(2^{6}\right),\left(4^{2}, 2^{2}\right)$ and $\left(6^{2}\right)$ - there are two $D_{6}$-classes (see [32, Lemma 3.11]), and the corresponding $E_{7}$-classes can be worked out by computing the dimension of $\mathbf{C}_{L\left(E_{7}\right)}(u)$ using the restriction $L\left(E_{7}\right) \downarrow D_{6}$ (see [32, Lemma 11.8]), where $L\left(E_{7}\right)$ denotes the Lie algebraa of $\mathcal{G}=E_{7}$. 


\begin{tabular}{|c|c|c|c|c|c|c|c|}
\hline$u$ in $D_{6}$ & $\left(2^{2}, 1^{8}\right)$ & $\left(3,1^{9}\right)$ & $\left(2^{4}, 1^{4}\right)$ & $\left(2^{6}\right)$ & $\left(2^{6}\right)^{\prime}$ & $\left(3,2^{2}, 1^{5}\right)$ & $\left(3,2^{4}, 1\right)$ \\
$u$ in $E_{7}$ & $A_{1}$ & $A_{1}^{2}$ & $A_{1}^{2}$ & $\left(A_{1}^{3}\right)^{(1)}$ & $\left(A_{1}^{3}\right)^{(2)}$ & $\left(A_{1}^{3}\right)^{(2)}$ & $A_{1}^{4}$ \\
$\operatorname{dim} u^{\mathcal{L}}$ & 18 & 20 & 28 & 30 & 30 & 32 & 36 \\
$\operatorname{dim} u^{\mathcal{G}}$ & 34 & 52 & 52 & 54 & 64 & 64 & 70 \\
\hline
\end{tabular}

\begin{tabular}{|c|c|c|c|c|c|c|c|}
\hline$\left(3,2,1^{6}\right)$ & $\left(3^{2}, 2^{2}, 1^{2}\right)$ & $\left(3^{3}, 1^{3}\right)$ & $\left(3^{4}\right)$ & $\left(4^{2}, 1^{4}\right)$ & $\left(5,1^{7}\right)$ & $\left(4^{2}, 2^{2}\right)$ & $\left(4^{2}, 2^{2}\right)^{\prime}$ \\
$A_{2}$ & $A_{2} A_{1}$ & $A_{2} A_{1}^{2}$ & $A_{2}^{2}$ & $A_{3}$ & $A_{3}$ & $\left(A_{3} A_{1}\right)^{(1)}$ & $\left(A_{3} A_{1}\right)^{(2)}$ \\
34 & 40 & 42 & 44 & 44 & 36 & 46 & 46 \\
66 & 76 & 82 & 84 & 84 & 84 & 86 & 92 \\
\hline
\end{tabular}

\begin{tabular}{|c|c|c|c|c|c|c|c|}
\hline$\left(5,2^{2}, 1^{3}\right)$ & $\left(4^{2}, 3,1\right)$ & $\left(5,3^{2}, 1\right)$ & $\left(5^{2}, 1^{2}\right)$ & $\left(6^{2}\right)$ & $\left(6^{2}\right)^{\prime}$ & $\left(7,1^{5}\right)$ & $\left(5,3,1^{4}\right)$ \\
$\left(A_{3} A_{1}\right)^{(1)}$ & $A_{3} A_{1}^{2}$ & $A_{3} A_{2}$ & $A_{4}$ & $\left(A_{5}\right)^{(1)}$ & $\left(A_{5}\right)^{(2)}$ & $D_{4}$ & $D_{4}\left(a_{1}\right)$ \\
44 & 48 & 50 & 52 & 54 & 54 & 48 & 46 \\
86 & 94 & 98 & 100 & 102 & 108 & 96 & 94 \\
\hline
\end{tabular}

\begin{tabular}{|c|c|c|c|c|c|c|}
\hline$\left(7,2^{2}, 1\right)$ & $\left(5,3,2^{2}\right)$ & $\left(9,1^{3}\right)$ & $\left(7,3,1^{2}\right)$ & $(11,1)$ & $(9,3)$, & $(7,5)$ \\
$D_{4} A_{1}$ & $D_{4}\left(a_{1}\right) A_{1}$ & $D_{5}$ & $D_{5}\left(a_{1}\right)$ & $D_{6}$ & $D_{6}\left(a_{1}\right)$ & $D_{6}\left(a_{2}\right)$ \\
52 & 48 & 56 & 54 & 60 & 58 & 56 \\
102 & 96 & 112 & 106 & 118 & 114 & 110 \\
\hline
\end{tabular}

Table 3 .

Inspecting the tables above, we see that the maximum value of $\operatorname{dim} u^{\mathcal{L}} / \operatorname{dim} u^{\mathcal{G}}$ is equal to $\frac{30}{54}$, and is attained when $u$ has Jordan form $\left(2^{6}\right)$ in $D_{6}$ and is in the class $\left(A_{1}^{3}\right)^{(1)}$ of $E_{7}$. Hence, for $\mathcal{L}^{\prime}=D_{6}$, we have $\alpha(\mathcal{L})=\frac{5}{9}$, as in Table 1 of Theorem 1.7.

Now, suppose that $\mathcal{L}$ is not one of the Levi subgroups listed for $\mathcal{G}=E_{7}$ in Table 1 (i.e. $\mathcal{L}$ does not have a factor $E_{6}, D_{r}$ or $A_{r}, r \geqslant 3$ ). Then, $\operatorname{dim} \mathcal{L} \leqslant \operatorname{dim} A_{2} A_{2} A_{1} T_{2}=21$.

Let $u$ be a non-trivial unipotent element in $\mathcal{L}$, and assume for a contradiction that

$$
\frac{\operatorname{dim} u^{\mathcal{L}}}{\operatorname{dim} u^{\mathcal{G}}}>\frac{1}{6} .
$$

Now, $\operatorname{dim} u^{\mathcal{L}} \leqslant \operatorname{dim} \mathcal{L}-7 \leqslant 14$, and hence $\operatorname{dim} u^{\mathcal{G}}<84$. It then follows from [32, Table 22.1.2] that $u$ is in one of the following $E_{7}$-classes:

$$
A_{1}, \quad A_{1}^{2}, \quad\left(A_{1}^{3}\right)^{(1)}, \quad\left(A_{1}^{3}\right)^{(2)}, \quad A_{1}^{4}, \quad A_{2}, \quad A_{2} A_{1}, \quad A_{2} A_{1}^{2} .
$$

For these classes the maximum possible value of $\operatorname{dim} u^{\mathcal{L}}$ occurs for $\mathcal{L}^{\prime}=A_{2} A_{2} A_{1}$ or $A_{1}^{4}$, and is as in Table 4

In all cases we see that $\operatorname{dim} u^{\mathcal{L}} / \operatorname{dim} u^{\mathcal{G}}<\frac{1}{6}$, which is a contradiction. This completes the proof of Theorem 1.7 for $\mathcal{G}=E_{7}$. 


\begin{tabular}{|c|c|c|c|c|c|c|c|c|}
\hline$u$ & $A_{1}$ & $A_{1}^{2}$ & $\left(A_{1}^{3}\right)^{(1)}$ & $\left(A_{1}^{3}\right)^{(2)}$ & $A_{1}^{4}$ & $A_{2}$ & $A_{2} A_{1}$ & $A_{2} A_{1}^{2}$ \\
\hline $\begin{array}{c}\max \operatorname{dim} u^{\mathcal{L}} \\
\mathcal{L}^{\prime}=A_{2} A_{2} A_{1}\end{array}$ & 4 & 8 & - & 10 & - & 6 & 10 & 12 \\
\hline $\begin{array}{c}\max \operatorname{dim} u^{\mathcal{L}} \\
\mathcal{L}^{\prime}=A_{1}^{4}\end{array}$ & 2 & 4 & 6 & 6 & 8 & - & - & - \\
\hline $\operatorname{dim} u^{\mathcal{G}}$ & 34 & 52 & 54 & 64 & 70 & 66 & 76 & 82 \\
\hline
\end{tabular}

Table 4.

\subsection{Proof of Corollary 1.8 and Theorem 1.9}

The proof of Corollary 1.8 is immediate, since

$$
1-\frac{1}{2} \frac{\operatorname{dim} y^{\mathcal{G}}}{\operatorname{dim} \mathcal{G}}=\frac{1}{2}\left(1+\frac{\operatorname{dim} \mathbf{C}_{\mathcal{G}}(y)}{\operatorname{dim} \mathcal{G}}\right)=\frac{1}{2}\left(1+\frac{\operatorname{dim} \mathcal{L}}{\operatorname{dim} \mathcal{G}}\right),
$$

and the right-hand side above is at least $\alpha(\mathcal{L})$, by Theorems 1.6 and 1.7.

To prove Theorem 1.9, note that $\chi(1) \geqslant \frac{1}{3} q^{r}$ by [24]. Also, $\mathbf{C}_{G}(g) \leqslant \mathbf{C}_{G}(y)=L$, and so, by Theorem 1.1 and the inequality $\alpha(L) \leqslant 1-\frac{1}{2} \operatorname{dim} y^{\mathcal{G}} / \operatorname{dim} \mathcal{G}$ obtained above, we have

$$
|\chi(g)| \leqslant f(r) \chi(1)^{1-\operatorname{dim} y^{\mathcal{G}} / 2 \operatorname{dim} \mathcal{G}} .
$$

Hence, it suffices to prove that $\gamma r \geqslant c s$, where $\gamma:=\operatorname{dim} y^{\mathcal{G}} / 2 \operatorname{dim} \mathcal{G}$ and $s:=\operatorname{supp}(y)$. Define $a:=1$ if $\mathcal{G}=\mathrm{SL}_{n}(\mathbb{K})$ and $a:=\frac{1}{2}$ otherwise.

Lemma 3.4 in [33] relates the support of elements of prime order in $G$ with the size of their conjugacy class. The proof of this lemma only uses the fact that these elements are semisimple or unipotent. Since $y \in \mathbf{Z}(L)$ is semisimple, the lemma applies and shows in particular that $\left|y^{G}\right| \geqslant c^{\prime} q^{a n s}$, where $c^{\prime}>0$ is an absolute constant. This implies that $\operatorname{dim} y^{\mathcal{G}} \geqslant$ ans, and so

$$
\gamma r \geqslant \frac{a n s}{2 \operatorname{dim} \mathcal{G}} r=\frac{a n r}{2 \operatorname{dim} \mathcal{G}} s=c s,
$$

as needed.

\subsection{Bounds for $\mathcal{G}=\mathrm{GL}_{n}(q)$ : Proofs of Theorem 1.10 and Corollary 1.11}

Let $\mathbb{K}$ an algebraically closed field of characteristic $p$, and let

$$
\mathcal{L}=\mathrm{GL}_{n_{1}}(\mathbb{K}) \times \ldots \times \mathrm{GL}_{n_{m}}(\mathbb{K})
$$


so that the Levi subgroup $L$ in Theorem 1.10 can be viewed as $\mathcal{L}^{F}$ for a suitable Frobenius endomorphism $F$. Fix $n$ pairwise distinct elements $\lambda_{1}, \ldots, \lambda_{n} \in \mathbb{K}^{\times}$. The statements follow from Theorem 1.1 if $n_{i_{0}}=1$, so we will assume that $n_{i_{0}} \geqslant 2$.

Any unipotent element $u \in L$ can be written $\operatorname{as} \operatorname{diag}\left(u_{1}, \ldots, u_{m}\right)$, where each $u_{i} \in \mathcal{L}_{i}:=$ $\mathrm{GL}_{n_{i}}(\mathbb{K})$ is unipotent. Write $u_{i}=J_{b_{i 1}} \oplus \ldots \oplus J_{b_{i r_{i}}}$ for a partition $\nu_{i}:=\left(b_{i 1} \geqslant b_{i 2} \geqslant \ldots \geqslant b_{i r_{i}} \geqslant 1\right)$ of $n_{i}$, and define

$$
s_{i}:=\operatorname{diag}\left(\lambda_{1}, \lambda_{2}, \ldots, \lambda_{b_{i 1}}, \lambda_{1}, \lambda_{2}, \ldots, \lambda_{b_{i 2}}, \ldots, \lambda_{1}, \lambda_{2}, \ldots, \lambda_{b_{i r_{i}}}\right) \in \mathcal{L}_{i} .
$$

Note that, if $a_{i j}$ is the multiplicity of $\lambda_{j}$ as an eigenvalue of $s_{i}$, then $\left(a_{i 1} \geqslant a_{i 2} \geqslant \ldots \geqslant a_{i n} \geqslant 0\right)$ is the partition of $n_{i}$ conjugate to $\nu_{i}$. Now, Lemma 4.1 shows that

$$
\operatorname{dim} u_{i}^{\mathcal{L}_{i}}=\operatorname{dim} s_{i}^{\mathcal{L}_{i}}=n_{i}^{2}-\sum_{j=1}^{n} a_{i j}^{2} .
$$

Similarly, setting $s:=\operatorname{diag}\left(s_{1}, \ldots, s_{m}\right) \in \mathcal{L}$, we then get

$$
\operatorname{dim} u^{\mathcal{G}}=\operatorname{dim} s^{\mathcal{G}}=n^{2}-\sum_{j=1}^{n}\left(\sum_{i=1}^{m} a_{i j}\right)^{2} .
$$

Note that $u \neq 1$ precisely when $\max _{1 \leqslant i \leqslant m} a_{i 2}>0$. Thus, $\alpha(L)=\beta\left(n_{1}, \ldots, n_{m}\right)$. Now, Theorem 1.10 follows immediately from Theorem 1.1 and Theorem 4.13 (i) below.

Note that, by the Cauchy-Schwartz inequality, $\left(\sum_{i=1}^{m} a_{i j}\right)^{2} \leqslant m \sum_{i=1}^{m} a_{i j}^{2}$ for each $j$, with equality attained exactly when $a_{1 j}=a_{2 j}=\ldots=a_{m j}$. Setting $\Delta:=\sum_{i, j} a_{i j}^{2}$, we have

$$
\beta\left(n_{1}, \ldots, n_{m}\right) \leqslant \max _{\Delta} \frac{\left(\sum_{i=1}^{m} n_{i}^{2}\right)-\Delta}{n^{2}-m \Delta} .
$$

Now, suppose that $n_{i}=n / m$ for $i=1, \ldots, m$. Then, $\sum_{i=1}^{m} n_{i}^{2} / n^{2}=1 / m$, and so (4.11) implies that $\beta\left(n_{1}, \ldots, n_{m}\right) \leqslant 1 / m$. In fact, equality holds if we choose $a_{1 j}=a_{2 j}=\ldots=a_{m j}$ for all $j$. Thus, Corollary 1.11 follows.

In what follows, for any partitions

$$
\alpha=\left(a_{1} \geqslant a_{2} \geqslant \ldots \geqslant a_{n} \geqslant 0\right) \vdash A \text { and } \beta=\left(b_{1} \geqslant b_{2} \geqslant \ldots \geqslant b_{n} \geqslant 0\right) \vdash B
$$

of $A, B \geqslant 1$, we set

$$
\begin{aligned}
g(\alpha) & :=A^{2}-\sum_{i=1}^{n} a_{i}^{2}, \\
h(\alpha) & :=\frac{g(\alpha)}{A}, \\
\alpha+\beta & :=\left(a_{1}+b_{1}, a_{2}+b_{2}, \ldots, a_{n}+b_{n}\right) \vdash(A+B) .
\end{aligned}
$$


LEMmA 4.12. Let $\alpha=\left(a_{1} \geqslant a_{2} \geqslant \ldots \geqslant a_{n} \geqslant 0\right) \vdash A$ and $\beta=\left(b_{1} \geqslant b_{2} \geqslant \ldots \geqslant b_{n} \geqslant 0\right) \vdash B$ be two partitions of $A, B \geqslant 1$. Then, $h(\alpha)+h(\beta) \leqslant h(\alpha+\beta)$.

Proof. We need to show that

$$
\frac{A^{2}-\sum_{i=1}^{n} a_{i}^{2}}{A}+\frac{B^{2}-\sum_{i=1}^{n} b_{i}^{2}}{B} \leqslant \frac{(A+B)^{2}-\sum_{i=1}^{n}\left(a_{i}+b_{i}\right)^{2}}{A+B},
$$

or, equivalently, that $\Gamma \geqslant 0$, where

$$
\begin{aligned}
\Gamma & :=A B \sum_{i \neq j}\left(a_{i}+b_{i}\right)\left(a_{j}+b_{j}\right)-(A+B)\left(B \sum_{i \neq j} a_{i} a_{j}+A \sum_{i \neq j} b_{i} b_{j}\right) \\
& =A B \sum_{i \neq j}\left(a_{i} b_{j}+a_{j} b_{i}\right)-\left(B^{2} \sum_{i \neq j} a_{i} a_{j}+A^{2} \sum_{i \neq j} b_{i} b_{j}\right) \\
& =A B\left(2 A B-2 \sum_{i} a_{i} b_{i}\right)-B^{2}\left(A^{2}-\sum_{i} a_{i}^{2}\right)-A^{2}\left(B^{2}-\sum_{i} b_{i}^{2}\right) \\
& =B^{2} \sum_{i} a_{i}^{2}+A^{2} \sum_{i} b_{i}^{2}-2 A B \sum_{i} a_{i} b_{i} .
\end{aligned}
$$

By the Cauchy-Schwarz inequality,

$$
2 A B \sum_{i} a_{i} b_{i} \leqslant 2 B\left(\sum_{i} a_{i}^{2}\right)^{1 / 2} A\left(\sum_{i} b_{i}^{2}\right)^{1 / 2} \leqslant B^{2} \sum_{i} a_{i}^{2}+A^{2} \sum_{i} b_{i}^{2},
$$

and the claim follows.

THEOREM 4.13. In the notation of Theorem 1.10, assume that

$$
n_{1}=n_{2}=\ldots=n_{t}>n_{t+1} \geqslant \ldots \geqslant n_{m} \geqslant 1 .
$$

Then, the following statements hold:

(i) $n_{1} / n \geqslant \beta\left(n_{1}, \ldots, n_{m}\right) \geqslant\left(n_{1}-1\right) /(n-t)$.

(ii) If $m=2$, then $\beta\left(n_{1}, n_{2}\right)=\left(n_{1}-1\right) /(n-t)$. Moreover, if

$$
1 \neq u=\operatorname{diag}\left(u_{1}, u_{2}\right) \in \mathrm{GL}_{n_{1}}(q) \times \mathrm{GL}_{n_{2}}(q)=L
$$

is a unipotent element, then $\left(\operatorname{dim} u^{\mathcal{L}}\right) /\left(\operatorname{dim} u^{\mathcal{G}}\right)=\alpha(L)$ precisely when one of the following conditions holds:

(a) $n_{1}=n_{2}$, and $u_{1}$ and $u_{2}$ have the same Jordan canonical form;

(b) $n_{1}>n_{2}, u_{1}$ is a transvection and $u_{2}=1$;

(c) $n_{1}=n_{2}+1$, and the sizes of Jordan blocks for $u_{1}$ and $u_{2}$ are

$$
c_{1} \geqslant \ldots \geqslant c_{j-1} \geqslant c_{j} \geqslant c_{j+1} \geqslant \ldots \geqslant c_{s} \text { and } c_{1} \geqslant \ldots \geqslant c_{j-1} \geqslant c_{j}-1 \geqslant c_{j+1} \geqslant \ldots \geqslant c_{s} \text {, }
$$

respectively. 
Proof. (i) To prove the lower bound for $\beta\left(n_{1}, \ldots, n_{m}\right)$, we choose $\left(a_{i 1}, \ldots, a_{i n}\right)$ to be $\left(n_{1}-1,1,0, \ldots, 0\right)$ if $1 \leqslant i \leqslant t$ and $\left(n_{i}, 0, \ldots, 0\right)$ otherwise. To prove the upper bound, for $1 \leqslant i \leqslant m$ consider the partition $\alpha_{i}:=\left(a_{i 1}, a_{i 2}, \ldots, a_{i n}\right) \vdash n_{i}$. By Lemma 4.12 , we have

$$
\sum_{i=1}^{m} \frac{g\left(\alpha_{i}\right)}{n_{i}}=h\left(\alpha_{1}\right)+h\left(\alpha_{2}\right)+\ldots+h\left(\alpha_{m}\right) \leqslant h\left(\sum_{i=1}^{m} \alpha_{i}\right)=\frac{g\left(\sum_{i=1}^{m} \alpha_{i}\right)}{n}
$$

in the notation of (4.12). Note that the condition $\max _{1 \leqslant i \leqslant m} a_{i 2}>0$ ensures that

$$
g\left(\sum_{i=1}^{m} \alpha_{i}\right)>0
$$

Since $n_{1}=\max _{1 \leqslant i \leqslant m} n_{i}$, (4.13) now implies that

$$
\beta\left(n_{1}, n_{2}, \ldots, n_{m}\right)=\frac{\sum_{i=1}^{m} g\left(\alpha_{i}\right)}{g\left(\sum_{i=1}^{m} \alpha_{i}\right)} \leqslant \frac{n_{1}\left(\sum_{i=1}^{m} g\left(\alpha_{i}\right) / n_{i}\right)}{g\left(\sum_{i=1}^{m} \alpha_{i}\right)} \leqslant \frac{n_{1}}{n} .
$$

(ii) We may assume $A:=n_{1}>B:=n_{2}$ by Corollary 1.11 and its proof. To ease the notation, also write

$$
\left(a_{11}, a_{12}, \ldots, a_{i n}\right)=\left(a_{1}, a_{2}, \ldots, a_{n}\right) \quad \text { and } \quad\left(a_{21}, a_{22}, \ldots, a_{2 n}\right)=\left(b_{1}, b_{2}, \ldots, b_{n}\right) .
$$

Then, we need to show that

$$
\frac{\sum_{1 \leqslant i \neq j \leqslant n}\left(a_{i} a_{j}+b_{i} b_{j}\right)}{\sum_{1 \leqslant i \neq j \leqslant n}\left(a_{i}+b_{i}\right)\left(a_{j}+b_{j}\right)} \leqslant \frac{A-1}{A+B-1},
$$

or, equivalently, that $\Sigma \geqslant 0$, where

$$
\begin{aligned}
\Sigma: & =(A-1) \sum_{i \neq j}\left(a_{i}+b_{i}\right)\left(a_{j}+b_{j}\right)-(A+B-1) \sum_{i \neq j}\left(a_{i} a_{j}+b_{i} b_{j}\right) \\
& =(A-1) \sum_{i \neq j}\left(a_{i} b_{j}+a_{j} b_{i}\right)-B \sum_{i \neq j}\left(a_{i} a_{j}+b_{i} b_{j}\right) \\
& =(A-1)\left(2 A B-2 \sum_{i} a_{i} b_{i}\right)-B\left(A^{2}+B^{2}-\sum_{i} a_{i}^{2}-\sum_{i} b_{i}^{2}\right) \\
& =B\left(A^{2}-2 A-B^{2}\right)+B\left(\sum_{i} a_{i}^{2}+\sum_{i} b_{i}^{2}\right)-2(A-1) \sum_{i} a_{i} b_{i} \\
& =B\left(A^{2}-2 A-B^{2}\right)+B \sum_{i}\left(a_{i}-b_{i}\right)^{2}-2(A-1-B) \sum_{i} a_{i} b_{i} \\
& =(A-1-B)\left((A-1+B) B-2 \sum_{i} a_{i} b_{i}\right)+B\left(\sum_{i}\left(a_{i}-b_{i}\right)^{2}-1\right) \\
& =(A-1-B)\left(\sum_{i}\left(A-1+B-2 a_{i}\right) b_{i}\right)+B\left(\sum_{i}\left(a_{i}-b_{i}\right)^{2}-1\right) .
\end{aligned}
$$


Note that the condition $A \geqslant B+1$ implies that $\sum_{i}\left(a_{i}-b_{i}\right)^{2} \geqslant 1$, with equality attained exactly when

$$
A=B+1 \quad \text { and } \quad\left(a_{1}, a_{2}, \ldots, a_{n}\right)=\left(b_{1}, \ldots, b_{i-1}, b_{i}+1, b_{i+1}, \ldots, b_{n}\right) .
$$

First, we consider the case when $A-1+B \geqslant 2 a_{i}$ for all $i$. As $B \geqslant 1$, we see that $\Sigma \geqslant 0$, with equality attained exactly when (4.14) holds, which means the corresponding unipotent element satisfies (c).

Suppose now that $A-1+B \leqslant 2 a_{i}-1$ for some $i$. Then, $a_{i} \geqslant \sum_{j \neq i} a_{j}+B$. As $B \geqslant 1$ and $a_{1} \geqslant a_{2} \geqslant \ldots \geqslant a_{n}$, this can happen only for one index $i$, and this index $i$ is 1 , and so

$$
a_{1} \geqslant A^{\prime}+B
$$

where $A^{\prime}:=\sum_{j \geqslant 2} a_{j}$. In particular, $A-1+B \geqslant 2 a_{j}$ for all $j \geqslant 2$. Now, by (4.15), we have

$$
\begin{aligned}
\Sigma^{\prime} & :=(A-1-B)\left(\left(A-1+b_{1}\right) b_{1}-2 a_{1} b_{1}\right)+b_{1}\left(a_{1}-b_{1}\right)^{2} \\
& =b_{1}\left((A-1-B)\left(b_{1}-1+A^{\prime}-a_{1}\right)+\left(a_{1}-b_{1}\right)^{2}\right) \\
& =b_{1}\left(\left(a_{1}-b_{1}\right)\left(A^{\prime}-1\right)+\left(A^{\prime}-1-B^{\prime}\right)\left(b_{1}-1+A^{\prime}-a_{1}\right)\right) \\
& =b_{1}\left(\left(a_{1}-b_{1}\right) B^{\prime}+\left(A^{\prime}-1\right)^{2}-B^{\prime}\left(A^{\prime}-1\right)\right) \\
& =b_{1}\left(\left(a_{1}-A^{\prime}-b_{1}+1\right) B^{\prime}+\left(A^{\prime}-1\right)^{2}\right) \geqslant 0,
\end{aligned}
$$

with equality exactly when

$$
B^{\prime}:=\sum_{j \geqslant 2} b_{j}=0 \quad \text { and } \quad A^{\prime}=1
$$

It follows that, if $\sum_{j \geqslant 2}\left(a_{j}-b_{j}\right)^{2} \geqslant 1$, then $\Sigma \geqslant 0$, with equality exactly when (4.16) holds, which means that the corresponding unipotent element satisfies (b).

Assume finally that $\sum_{j \geqslant 2}\left(a_{j}-b_{j}\right)^{2} \leqslant 0$. Then, $a_{j}=b_{j}$ for all $j \geqslant 2$ and $A^{\prime}=B^{\prime}$. As $\max \left(a_{2}, b_{2}\right)>0$, we must have $A^{\prime}=B^{\prime} \geqslant 1$, and so, by (4.15),

$$
\Sigma^{\prime}=b_{1}\left(\left(a_{1}-b_{1}-1\right) A^{\prime}+1\right) \geqslant 2 b_{1},
$$

yielding $\Sigma \geqslant b_{1} \geqslant 1$.

We note that Theorem 4.13 (i) was inspired by some correspondence with M. Fraczyk, who is studying the situation in Theorem 1.10 using different methods. 


\section{Random walks}

In this section we prove Theorems $1.12-1.15$ concerning random walks and covering numbers.

Proof of Theorem 1.12. Suppose $\mathcal{G}$ is a simple algebraic group of rank $r$ in good characteristic, and $G=G(q)=\mathcal{G}^{F}$ is a finite quasisimple group over $\mathbb{F}_{q}$. Let $y \in G$ be such that $\mathbf{C}_{G}(y) \leqslant L=\mathcal{L}^{F}$ for a proper split Levi subgroup $\mathcal{L}$ of $\mathcal{G}$. Write $C=y^{G}$, and let $h$ be the Coxeter number of $\mathcal{G}$.

For a real number $s$, define

$$
\zeta^{G}(s)=\sum_{\chi \in \operatorname{Irr}(G)} \chi(1)^{-s}
$$

We will need the following result, which is [36, Theorem 1.1].

LEMMA 5.1. If $s>2 / h$, then $\zeta^{G}(s) \rightarrow 1$ as $q \rightarrow \infty$.

We first prove part (I) (a) of Theorem 1.12 together with the first statement of part (II) (the $C^{6}=G$ statement). We will prove the mixing time assertions later.

Let $t$ be a positive integer. By a well-known result (see [2, Chapter 1, Lemma 10.1]), for $g \in G$ the number of ways of writing $g$ as a product of $t$ conjugates of $y$ is

$$
N(g)=\frac{|C|^{t}}{|G|} \sum_{\chi \in \operatorname{Irr}(G)} \frac{\chi(y)^{t} \chi\left(g^{-1}\right)}{\chi(1)^{t-1}} .
$$

Define $P^{t}(g)=N(g) /|C|^{t}$, the probability that a random product of $t$ conjugates of $y$ is equal to $g$, and let $U(g)=1 /|G|$, the uniform probability distribution on $G$. Then,

$$
\left|P^{t}(g)-U(g)\right| \leqslant \frac{1}{|G|} \sum_{\chi(1)>1}\left(\frac{|\chi(y)|}{\chi(1)}\right)^{t} \chi(1)^{2} .
$$

Define

$$
\left\|P^{t}-U\right\|_{\infty}=|G| \max _{g \in G}\left|P^{t}(g)-U(g)\right| .
$$

Write $\alpha=\alpha(\mathcal{L})$. Then, Theorem 1.1 gives

$$
\frac{|\chi(y)|}{\chi(1)} \leqslant f(r) \chi(1)^{\alpha-1}
$$

and so (5.2) implies that

$$
\left\|P^{t}-U\right\|_{\infty} \leqslant f(r)^{t} \sum_{\chi(1)>1} \chi(1)^{t(\alpha-1)+2}=f(r)^{t}\left(\zeta^{G}(t(1-\alpha)-2)-1\right) .
$$


By Lemma 5.1, $\zeta^{G}(t(1-\alpha)-2)-1 \rightarrow 0$ as $q \rightarrow \infty$, provided

$$
t(1-\alpha)-2>\frac{2}{h}
$$

If $\mathcal{G}$ is of exceptional type $G_{2}, F_{4}, E_{6}, E_{7}$ or $E_{8}$, then $2 / h$ is $\frac{1}{3}, \frac{1}{6}, \frac{1}{6}, \frac{1}{9}$ or $\frac{1}{15}$, respectively, and Theorem 1.7 shows that (5.3) holds in all cases, provided $t \geqslant 6$. This proves the first statement of Theorem 1.12 (II).

Now suppose that $\mathcal{G}$ is of classical type. Then,

$$
\alpha \leqslant \frac{1}{2}\left(1+\frac{\operatorname{dim} \mathcal{L}}{\operatorname{dim} \mathcal{G}}\right)
$$

by Theorem 1.6. This implies that (5.3) holds, provided

$$
t>\left(4+\frac{4}{h}\right) \frac{\operatorname{dim} \mathcal{G}}{\operatorname{dim} \mathcal{G}-\operatorname{dim} \mathcal{L}}
$$

proving Theorem $1.12(\mathrm{I})(\mathrm{a})$.

We now prove the assertions on mixing times in Theorem 1.12. For these, we use the Diaconis-Shashahani bound [6]:

$$
\left(\left\|P^{t}-U\right\|_{1}\right)^{2} \leqslant \sum_{\substack{\chi \in \operatorname{Irr}(G) \\ \chi \neq 1}}\left(\frac{|\chi(y)|}{\chi(1)}\right)^{2 t} \chi(1)^{2} .
$$

As above, Theorem 1.1 shows that the right-hand side of (5.4) is less than

$$
f(r)^{2 t}\left(\zeta^{G}(2 t(1-\alpha)-2)-1\right)
$$

and hence tends to zero as $q \rightarrow \infty$, provided $2 t(1-\alpha)-2>2 / h$. Using Theorems 1.7 and 1.6, we now see, as before, that this inequality holds provided $t \geqslant 3$ when $\mathcal{G}$ is of exceptional type, and provided

$$
t>\left(2+\frac{2}{h}\right) \frac{\operatorname{dim} \mathcal{G}}{\operatorname{dim} \mathcal{G}-\operatorname{dim} \mathcal{L}}
$$

when $\mathcal{G}$ is classical. This proves the mixing time assertions, completing the proof of Theorem 1.12 .

Proof of Theorem 1.15. This is very similar to the previous proof, using Corollary 3.5 instead of Theorem 1.1. Let $G=\mathrm{SL}_{n}(q)$ and let $u \in G$ be a non-identity unipotent 
element. Let $t \in \mathbb{N}$, and for $g \in G$ let $P^{t}(g)$ be the probability that a random product of $t$ conjugates of $u$ is equal to $g$, and $U(g)=1 /|G|$. As in (5.2),

$$
\left|P^{t}(g)-U(g)\right| \leqslant \frac{1}{|G|} \sum_{\substack{\chi \in \operatorname{Irr}(G) \\ \chi(1)>1}}\left(\frac{|\chi(u)|}{\chi(1)}\right)^{t} \chi(1)^{2} .
$$

By Corollary 3.5,

$$
\frac{|\chi(u)|}{\chi(1)} \leqslant g(n) \chi(1)^{-1 /(n-1)}
$$

for $\chi \in \operatorname{Irr}(G)$, and hence

$$
\left\|P^{t}-U\right\|_{\infty} \leqslant g(n)^{t} \sum_{\chi(1)>1} \chi(1)^{-t /(n-1)+2}=g(n)^{t}\left(\zeta^{G}\left(\frac{t}{n-1}-2\right)-1\right) .
$$

By Lemma 5.1, $\zeta^{G}(t /(n-1)-2)-1 \rightarrow 0$ as $q \rightarrow \infty$ provided $t /(n-1)-2>2 / n$, which holds provided $t>2 n$. This proves part (i) of Theorem 1.15. Part (ii) is proved in the same way, using the bound (5.4).

\section{Corollaries 1.13 and 1.14}

Corollary 1.13 follows immediately from Theorem 1.12 (I) (b). Corollary 1.14 is proved exactly as above, using Theorem 1.5.

Next, we use some well-known observations to justify the remarks made after the statement of Theorem 1.12.

LEMma 5.2. (i) Let $G$ be a finite group, and let $S$ be a generating subset of $G$ that satisfies $\left|S^{N}\right|<|G|(1-1 / e)$ for some integer $N \geqslant 1$. Then, the mixing time $T(G, S)$ of the random walk on the Cayley graph corresponding to $S$ is at least $N+1$.

(ii) Let $G=\operatorname{SL}_{n}(q)$, with $n \geqslant 2$, and $S=y^{G}$, with $y=\operatorname{diag}\left(\mu I_{n-1}, \lambda\right)$, where $\mu, \lambda \in \mathbb{F}_{q}^{\times}$ and $\mu \neq \lambda$. Then, $T(G, y) \geqslant n$.

Proof. (i) Define $P(g)$ to be $1 /|S|$ if $g \in S$ and zero otherwise, and let $U(g)=1 /|G|$ for all $g \in G$. Consider any $1 \leqslant k \leqslant N$. Note that $\left|S^{k}\right| \leqslant\left|S^{k+1}\right|$, and so $\left|S^{k}\right| \leqslant\left|S^{N}\right|$, whence

$$
\left\|P^{k}-U\right\|_{1} \geqslant \sum_{g \in G \backslash S^{k}}\left|P^{k}(g)-U(g)\right|=\sum_{g \in G \backslash S^{k}}|U(g)| \geqslant \frac{\left|G \backslash S^{k}\right|}{|G|}>\frac{1}{e} .
$$

It follows that $T(G, S) \geqslant N+1$. 
(ii) Note that $\left(y^{G}\right)^{n-1}$ is contained in $X$, the set of elements $x \in G$ that have eigenvalue $\mu$ on $V=\mathbb{F}_{q}^{n}$. Now, if we fix $0 \neq v \in V$ and let $Y:=\{x \in G: x(v)=\mu v\}$, then it is easy to see that $\left|\mathbf{N}_{G}(Y)\right| /|Y| \geqslant q-1 \geqslant 2$. Hence,

$$
|X|=\left|\bigcup_{g \in G} g Y g^{-1}\right| \leqslant|Y|\left[G: \mathbf{N}_{G}(Y)\right] \leqslant \frac{|G|}{2} .
$$

Now we can apply (i) to $S:=y^{G}$.

We conclude with a proof of our last theorem, connecting the mixing times of random walks on classical groups with the support of certain elements.

Proof of Theorem 1.17. Set $s:=\operatorname{supp}(y)$. Then,

$$
\mathbf{C}_{G}(g) \leqslant \mathbf{C}_{G}(y)=L \text {. }
$$

Theorem $1.12(\mathrm{I})(\mathrm{b})$ gives

$$
T(G, g) \leqslant\left\lceil\left(2+\frac{2}{h}\right) \frac{\operatorname{dim} \mathcal{G}}{\operatorname{dim} \mathcal{G}-\operatorname{dim} \mathcal{L}}\right\rceil
$$

for large $q$. Now, $\operatorname{dim} \mathcal{G}-\operatorname{dim} \mathcal{L}=\operatorname{dim} \mathcal{G}-\operatorname{dim} \mathbf{C}_{\mathcal{G}}(y)=\operatorname{dim} y^{\mathcal{G}} \geqslant a n s$, as shown in the proof of Theorem 1.9. This yields

$$
T(G, g) \leqslant\left\lceil\left(2+\frac{2}{h}\right) \frac{\operatorname{dim} \mathcal{G}}{\text { ans }}\right\rceil .
$$

Let $c=c(\mathcal{G})$ be as in Theorem 1.9. Then, we have $(\operatorname{dim} \mathcal{G}) / a n=r / c=r^{\prime}$. We obtain

$$
T(G, g) \leqslant\left\lceil\left(2+\frac{2}{h}\right) \frac{r^{\prime}}{s}\right\rceil,
$$

proving the first assertion.

It remains to prove the lower bound on $T(G, y)$. By $(1.3)$, we have

$$
T(G, y) \geqslant \frac{\log |G|+\log \left(1-e^{-1}\right)}{\log \left|y^{G}\right|} \gtrsim_{|G|} \frac{\operatorname{dim} \mathcal{G}}{\operatorname{dim} y^{\mathcal{G}}} .
$$

It follows from [33, Lemma 3.4] and its proof that, for $y$ semisimple, we have $\left|y^{G}\right| \leqslant 2$ ans. Hence, $\operatorname{dim} y^{\mathcal{G}} \leqslant 2$ ans, which, combined with the inequality above, implies

$$
T(G, y) \gtrsim_{|G|} \frac{\operatorname{dim} \mathcal{G}}{2 a n s} \geqslant \frac{\operatorname{dim} \mathcal{G}}{2 a n} \frac{1}{s}=\frac{1}{2} \frac{r^{\prime}}{s},
$$

as required.

Acknowledgements. Parts of the paper were written while the fourth author visited the Departments of Mathematics of Imperial College, London, and University of Chicago. It is a pleasure to thank Imperial College, Prof. Ngo Bao Chau, and the University of Chicago for generous hospitality and stimulating environment. The authors also thank M. Geck, G. Malle, J. Michel, and especially J. Taylor for many helpful discussions. The authors are grateful to the referees for careful reading and helpful comments on the paper. 


\section{References}

[1] Achar, P. N. \& Aubert, A.-M., Supports unipotents de faisceaux caractères. J. Inst. Math. Jussieu, 6 (2007), 173-207.

[2] Arad, Z., Herzog, M. \& Stavi, J., Powers and products of conjugacy classes in groups, in Products of Conjugacy Classes in Groups, Lecture Notes in Math., 1112, pp. 6-51. Springer, Berlin-Heidelberg, 1985.

[3] Bourbaki, N., Éléments de mathématique. Fasc. XXXIV. Groupes et algèbres de Lie. (Chapters 4-6). Actualités Scientifiques et Industrielles, 1337. Hermann, Paris, 1968.

[4] Brundan, J. \& Kleshchev, A., Lower bounds for degrees of irreducible Brauer characters of finite general linear groups. J. Algebra, 223 (2000), 615-629.

[5] Carter, R. W., Finite Groups of Lie Type: Conjugacy Classes and Complex Characters. Pure and Applied Mathematics. Wiley, New York, 1985.

[6] Diaconis, P. \& Shahshahani, M., Generating a random permutation with random transpositions. Z. Wahrsch. Verw. Gebiete, 57 (1981), 159-179.

[7] Digne, F., Lehrer, G. \& Michel, J., On character sheaves and characters of reductive groups at unipotent classes. Pure Appl. Math. Q., 10 (2014), 459-512.

[8] Digne, F. \& Michel, J., Representations of Finite Groups of Lie Type. London Mathematical Society Student Texts, 21. Cambridge Univ. Press, Cambridge, 1991.

[9] Fomin, S. \& Lulov, N., On the number of rim hook tableaux. Zap. Nauchn. Sem. S.Peterburg. Otdel. Mat. Inst. Steklov. (POMI), 223 (1995), 219-226, 340 (Russian); English translation in J. Math. Sci. (New York), 87 (1997), 4118-4123.

[10] Frobenius, F. G., Über Gruppencharaktere. Sitzber. Preuss. Akad. Wiss., (1896), 9851021. Reprinted in Gesammelte Abhandlungen, Vol. 3, pp.1-37. Springer, BerlinHeidelberg, 1968.

[11] GECK, M., On the average values of the irreducible characters of finite groups of Lie type on geometric unipotent classes. Doc. Math., 1 (1996), 293-317.

[12] Geck, M., Hiss, G. \& Malle, G., Cuspidal unipotent Brauer characters. J. Algebra, 168 (1994), 182-220.

[13] GuucK, D., Character value estimates for nonsemisimple elements. J. Algebra, 155 (1993), 221-237.

[14] - Characters and random walks on finite classical groups. Adv. Math., 129 (1997), 46-72.

[15] Green, J. A., The characters of the finite general linear groups. Trans. Amer. Math. Soc., 80 (1955), 402-447.

[16] Guralnick, R. M., Larsen, M. \& Tiep, P. H., Character levels and character bounds. Preprint, 2017. arXiv:1708.03844 [math.RT].

[17] Hildebrand, M., Generating random elements in $\mathrm{SL}_{n}\left(\mathbf{F}_{q}\right)$ by random transvections. $J$. Algebraic Combin., 1 (1992), 133-150.

[18] Howlett, R. B. \& Lehrer, G. I., Representations of generic algebras and finite groups of Lie type. Trans. Amer. Math. Soc., 280 (1983), 753-779.

[19] Humphreys, J. E., Conjugacy Classes in Semisimple Algebraic Groups. Mathematical Surveys and Monographs, 43. Amer. Math. Soc., Providence, RI, 1995.

[20] IsaACS, I. M., Character Theory of Finite Groups. AMS Chelsea, Providence, RI, 2006.

[21] James, G. \& Kerber, A., The Representation Theory of the Symmetric Group. Encyclopedia of Mathematics and its Applications, 16. Addison-Wesley, Reading, MA, 1981.

[22] Kawanaka, N., Generalized Gel'fand-Graev representations of exceptional simple algebraic groups over a finite field. I. Invent. Math., 84 (1986), 575-616.

[23] Kleshchev, A. S. \& TieP, P. H., Representations of the general linear groups which are irreducible over subgroups. Amer. J. Math., 132 (2010), 425-473. 
[24] Landazuri, V. \& Seitz, G. M., On the minimal degrees of projective representations of the finite Chevalley groups. J. Algebra, 32 (1974), 418-443.

[25] Larsen, M. \& Shalev, A., Characters of symmetric groups: sharp bounds and applications. Invent. Math., 174 (2008), 645-687.

[26] Larsen, M., Shalev, A. \& TieP, P. H., The Waring problem for finite simple groups. Ann. of Math., 174 (2011), 1885-1950.

[27] Lawther, R. \& Liebeck, M. W., On the diameter of a Cayley graph of a simple group of Lie type based on a conjugacy class. J. Combin. Theory Ser. A, 83 (1998), 118-137.

[28] LEv, A., The covering number of the group $\mathrm{PSL}_{n}(F)$. J. Algebra, 182 (1996), 60-84.

[29] Liebeck, M. W., Character ratios for finite groups of Lie type, and applications, in Finite Simple Groups: Thirty Years of the Atlas and Beyond, Contemp. Math., 694, pp. 193208. Amer. Math. Soc., Providence, RI, 2017.

[30] Liebeck, M. W., O’Brien, E. A., Shalev, A. \& Tiep, P. H., The Ore conjecture. J. Eur. Math. Soc. (JEMS), 12 (2010), 939-1008.

[31] Liebeck, M. W., Schul, G. \& Shalev, A., Rapid growth in finite simple groups. Trans. Amer. Math. Soc., 369 (2017), 8765-8779.

[32] Liebeck, M. W. \& Seitz, G. M., Unipotent and Nilpotent Classes in Simple Algebraic Groups and Lie Algebras. Mathematical Surveys and Monographs, 180. Amer. Math. Soc., Providence, RI, 2012.

[33] Liebeck, M. W. \& Shalev, A., Simple groups, permutation groups, and probability. J. Amer. Math. Soc., 12 (1999), 497-520.

[34] — Diameters of finite simple groups: sharp bounds and applications. Ann. of Math., 154 (2001), 383-406.

[35] — Fuchsian groups, coverings of Riemann surfaces, subgroup growth, random quotients and random walks. J. Algebra, 276 (2004), 552-601.

[36] - Character degrees and random walks in finite groups of Lie type. Proc. London Math. Soc., 90 (2005), 61-86.

[37] Liebeck, M. W., Shalev, A. \& Tiep, P.H., Character ratios, representation varieties and random generation of finite groups of Lie type. Preprint, 2017. arXiv: 1807.08842 [math.GR].

[38] Logan, B. F. \& Shepp, L. A., A variational problem for random Young tableaux. Adv. Math., 26 (1977), 206-222.

[39] Lubotzky, A., Cayley graphs: eigenvalues, expanders and random walks, in Surveys in Combinatorics, 1995 (Stirling), London Math. Soc. Lecture Note Ser., 218, pp. 155-189. Cambridge Univ. Press, Cambridge, 1995.

[40] Lulov, N. \& PAK, I., Rapidly mixing random walks and bounds on characters of the symmetric group. J. Algebraic Combin., 16 (2002), 151-163.

[41] Lusztig, G., Characters of Reductive Groups over a Finite Field. Annals of Mathematics Studies, 107. Princeton Univ. Press, Princeton, NJ, 1984.

[42] - Green functions and character sheaves. Ann. of Math., 131 (1990), 355-408.

[43] Roichman, Y., Upper bound on the characters of the symmetric groups. Invent. Math., 125 (1996), 451-485.

[44] Shalev, A., Mixing and generation in simple groups. J. Algebra, 319 (2008), 3075-3086.

[45] - Word maps, conjugacy classes, and a noncommutative Waring-type theorem. Ann. of Math., 170 (2009), 1383-1416.

[46] - Conjugacy classes, growth and complexity, in Finite Simple Groups: Thirty Years of the Atlas and Beyond, Contemp. Math., 694, pp. 209-221. Amer. Math. Soc., Providence, RI, 2017. 
[47] Shoji, T., Character sheaves and almost characters of reductive groups. I, II. Adv. Math., 111 (1995), 244-313, 314-354.

[48] - On the computation of unipotent characters of finite classical groups. Appl. Algebra Engrg. Comm. Comput., 7 (1996), 165-174.

[49] Steinberg, R., The representations of GL(3, $q)$, GL $(4, q), \operatorname{PGL}(3, q)$, and $\operatorname{PGL}(4, q)$. Canadian J. Math., 3 (1951), 225-235.

[50] TAYLOR, J., Generalized Gel'fand-Graev representations in small characteristics. Nagoya Math. J., 224 (2016), 93-167.

[51] TieP, P. H., Weil representations of finite general linear groups and finite special linear groups. Pacific J. Math., 279 (2015), 481-498.

[52] Tiep, P. H. \& Zalesskit, A. E., Minimal characters of the finite classical groups. Comm. Algebra, 24 (1996), 2093-2167.

[53] Vershik, A. M. \& Kerov, S. V., Asymptotic behavior of the Plancherel measure of the symmetric group and the limit form of Young tableaux. Dokl. Akad. Nauk SSSR, 233 (1977), 1024-1027 (Russian); English translation in Soviet Math. Dokl., 233 (1977), 527-531.

[54] Vishne, U., Mixing and covering in the symmetric groups. J. Algebra, 205 (1998), 119-140.

Roman BezRukavnikov

Department of Mathematics

Massachusetts Institute of Technology

Cambridge, MA 02139

U.S.A.

bezrukav@math.mit.edu

Aner Shalev

Institute of Mathematics

Hebrew University

Jerusalem 91904

Israel

shalev@math.huji.ac.il

\author{
Martin W. Liebeck \\ Department of Mathematics \\ Imperial College \\ London SW7 2BZ \\ U.K. \\ m.liebeck@imperial.ac.uk \\ Pham HuU TieP \\ Department of Mathematics \\ Rutgers University \\ Piscataway, NJ 08854 \\ U.S.A. \\ tiep@math .rutgers . edu
}

Received November 2, 2017

Received in revised form August 7, 2018 